\title{
Hilbert functions of points on Schubert varieties in orthogonal Grassmannians
}

\author{
K.N. Raghavan • Shyamashree Upadhyay
}

Received: 2 October 2008 / Accepted: 3 June 2009 / Published online: 19 June 2009

(C) Springer Science+Business Media, LLC 2009

\begin{abstract}
Given a point on a Schubert variety in an orthogonal Grassmannian, we compute the multiplicity, more generally the Hilbert function. We first translate the problem from geometry to combinatorics by applying standard monomial theory. The solution of the resulting combinatorial problem forms the bulk of the paper. This approach has been followed earlier to solve the same problem for Grassmannians and symplectic Grassmannians.

As an application, we present an interpretation of the multiplicity as the number of non-intersecting lattice paths of a certain kind. A more important application, although it does not appear here but elsewhere, is to the computation of the initial ideal, with respect to certain convenient monomial orders, of the ideal of the tangent cone to the Schubert variety.

Taking the Schubert variety to be of a special kind and the point to be the 'identity coset,' our problem specializes to one about Pfaffian ideals, treatments of which by different methods exist in the literature. Also available in the literature is a geometric solution when the point is a 'generic singularity.'
\end{abstract}

Keywords Orthogonal Grassmannian · Schubert variety · Hilbert function · Multiplicity · Pfaffian ideal · O-domination · O-depth

\section{Introduction}

Given a Schubert variety in an orthogonal Grassmannian (by which is meant the variety of isotropic subspaces of maximum possible dimension of a finite dimensional

K.N. Raghavan $(\bowtie)$

Institute of Mathematical Sciences, C. I. T. Campus, Chennai 600113, India

e-mail: knr@imsc.res.in

S. Upadhyay

Chennai Mathematical Institute, Plot No. H1, SIPCOT IT Park Padur Post, Siruseri 603103,

Tamilnadu, India

e-mail: shyama@cmi.ac.in 
vector space with a symmetric non-degenerate form-see $\S 2$ for precise definitions) and an arbitrary point on the Schubert variety, we compute the multiplicity, more generally the Hilbert function, of the local ring of germs of functions at that point. More precisely, we give a combinatorial description of the Hilbert function (Theorem 2.5.1) which enables us to interpret the multiplicity as the number of certain non-intersecting lattice paths $(\$ 3)$ and to compute the initial ideal of the tangent cone to the Schubert variety ([18]).

The analogous treatment of Grassmannians appears in [7-9, 11, 12] and of symplectic Grassmannians in [4]. The present paper is a sequel to [4, 7, 9, 11, 12] and toes the same line as they do. In particular, its strategy is borrowed from them and runs as follows: first translate the problem from geometry to combinatorics, or, more precisely, apply standard monomial theory to obtain an initial combinatorial description of the Hilbert function (the earliest version of the theory capable of handling Schubert varieties in an orthogonal Grassmannian is to be found in [19]); then transform the initial combinatorial description to obtain the desired alternative description. But all this is easier said than done.

While the problem makes sense for Schubert varieties of any kind and standard monomial theory itself is available in great generality [14, 16, 17], the translation of the problem from geometry to combinatorics has been made — in [15] — only for 'minuscule $^{1}$ generalized Grassmannians.' Orthogonal Grassmannians being minuscule, this translation is available to us and we have an initial combinatorial description of the Hilbert function. As to the passage from the initial to the alternative descriptionand this is where the content of the present paper lies-neither the end nor the means is clear at the outset.

The first problem then is to find a good alternative description. But how to measure the worth of an alternative description? The interpretation of multiplicity as the number of certain non-intersecting lattice paths, deduced in $\$ 3$ from our alternative description, seems to testify to its correctness. An even stronger justification is that it enables, as shown in [18], computation of Gröbner degenerations of tangent cones at torus fixed points to Schubert varieties.

The proof of the equivalence of the initial and alternative combinatorial descriptions is, unfortunately, a little technically involved. It builds on the details of the proofs of the corresponding equivalences in the cases of the Grassmannian and the symplectic Grassmannian. In [10] it is shown that the equivalence in the case of the Grassmannian is a kind of KRS correspondence, called 'bounded KRS.' The proof there is short and elegant and it would be nice to realize the main result of the present paper too in a similar spirit as a kind of KRS correspondence.

Taking the Schubert variety to be of a special kind and the point to be the 'identity coset,' our problem specializes to one about Pfaffian ideals considered in [2, 5]. On the other side of the spectrum from the identity coset, so to speak, are points that are generic in the complement of the open orbit of the stabiliser of the Schubert variety. For these, a geometric solution to the problem appears in [1].

${ }^{1}$ Symplectic Grassmannians are not minuscule but can be treated as if they were. 
One can ask if it is possible to extract more tangible information-closed form formulas for example-from our alternative description, although our interest is not so much along this line as in computing Gröbner degenerations. The papers quoted in the previous paragraph and [3] give some answers in the special cases they consider. More interestingly, the recent article [6] gives not only an interpretation of the multiplicity similar to ours but also a closed form formula. But it is not clear that the approach of [6] leads to Gröbner degenerations or for that matter to the Hilbert function: the point of [6] is to circumvent the need for Gröbner degenerations (precursors of [6] in the Grassmannian and symplectic Grassmannian cases having had these as starting points), while ours is to arrive at them. In any case, [6] is different in both method and the nature of results relied upon.

The organization of the paper is as follows: the set up is described and the main theorem stated in $\S 2$; the interpretation of multiplicity is given in $\S 3$; the proof of the main theorem is reduced to those of certain combinatorial statements in $\S 4$; the proof of these statements forms the bulk of the paper: it is eventually given in $\$ 9$ after preparations in $\$ 5-8$. The appearance of the tombstone ( $\square$ ) symbol immediately after an assertion means that the proof is easy and hence omitted.

We thank the referees for comments aimed at improving readability. In particular, the inclusion of examples is at their behest.

\section{The set up and the theorem}

\subsection{Initial statement of the problem}

Fix once for all a base field $\mathfrak{k}$, algebraically closed and of characteristic not 2 ; a natural number $d$; a vector space $V$ of dimension $2 d$ with a non-degenerate symmetric bilinear form $\langle$,$\rangle ; and a basis e_{1}, \ldots, e_{2 d}$ of $V$ such that $\left\langle e_{i}, e_{k}\right\rangle$ is 1 if $i=k^{*}$ and 0 otherwise, where $k^{*}:=2 d+1-k$.

Denote by $\mathrm{SO}(V)$ the group of linear automorphisms of $V$ preserving $\langle$,$\rangle and the$ volume form. Denote by $\mathfrak{M}_{d}(V)^{\prime}$ the closed sub-variety of the Grassmannian of $d$ dimensional subspaces consisting of the points corresponding to isotropic subspaces. The action of $\mathrm{SO}(V)$ on $V$ induces an action on $\mathfrak{M}_{d}(V)^{\prime}$. There are two orbits for this action. They are isomorphic: acting by a linear automorphism preserving the form but not the volume form gives an isomorphism. Denote by $\mathfrak{M}_{d}(V)$ the orbit of the span of $e_{1}, \ldots, e_{d}$ and call it the (even) orthogonal Grassmannian.

The Schubert varieties of $\mathfrak{M}_{d}(V)$ are defined to be the $B$-orbit closures in $\mathfrak{M}_{d}(V)$ (with canonical reduced scheme structure), where $B$ is a Borel subgroup of $\operatorname{SO}(V)$. The problem that is tackled in this paper is this: given a point on a Schubert variety in $\mathfrak{M}_{d}(V)$, compute the multiplicity, or more generally the Hilbert function, of the local ring of germs of functions at that point. The solutions are to be found in Theorem 2.5.1, Corollary 2.5.2, and in $\$ 3$. 
Orthogonal Grassmannians and their Schubert varieties are, of course, also defined when the dimension of $V$ is odd. As is well known and recalled with proof in [20, $\S 1.3]$, such Schubert varieties are isomorphic to those in even orthogonal Grassmannians. So the results of this paper would apply also to them.

\subsection{The problem restated}

We take $B$ to be the subgroup consisting of elements upper triangular with respect to $e_{1}, \ldots, e_{2 d}$. The subgroup $T$ of elements diagonal with respect to $e_{1}, \ldots, e_{2 d}$ is a maximal torus of $\mathrm{SO}(V)$. The $B$-orbits of $\mathfrak{M}_{d}(V)$ are naturally indexed by its $T$-fixed points: each orbit contains a unique such point. The $T$-fixed points are easily seen to be of the form $\left\langle e_{i_{1}}, \ldots, e_{i_{d}}\right\rangle$ for $\left\{i_{1}, \ldots, i_{d}\right\}$ in $\mathfrak{O} I(d)$, where $\mathfrak{O} I(d)$ is the set of subsets of $\{1, \ldots, 2 d\}$ of cardinality $d$ satisfying:

- for each $k, 1 \leq k \leq d$, there does not exist $j, 1 \leq j \leq d$, such that $i_{k}^{*}=i_{j}$-in other words, for each $\ell, 1 \leq \ell \leq 2 d$, exactly one of $\ell$ and $\ell^{*}$ appears in $\left\{i_{1}, \ldots, i_{d}\right\}$;

- the parity is even of the number of elements of the subset that are (strictly) greater than $d$.

Let $I(d, 2 d)$ denote the set of all subsets of cardinality $d$ of $\{1, \ldots, 2 d\}$. We use symbols $v, w, \ldots$ to denote elements of $I(d, 2 d)$ (in particular, those of $\mathfrak{O} I(d))$. The members of $v$ are denoted $v_{1}, \ldots, v_{d}$, with the convention that $1 \leq v_{1}<\ldots<v_{d} \leq$ $2 d$. There is a natural partial order on $I(d, 2 d): v \leq w$, if $v_{1} \leq w_{1}, \ldots, v_{d} \leq w_{d}$.

The point of the orthogonal Grassmannian $\mathfrak{M}_{d}(V)$ that is the span of $e_{v_{1}}, \ldots$, $e_{v_{d}}$ for $v \in \mathfrak{O} I(d)$ is denoted $\mathfrak{e}(v)$. The $B$-orbit closure of $\mathfrak{e}(v)$ is denoted $X^{v}$. The point $\mathfrak{e}(v)$ (and therefore the Schubert variety $X^{v}$ ) is contained in the Schubert variety $X^{w}$ if and only if $v \leq w$.

Our problem can now be stated thus: given elements $v \leq w$ of $\mathfrak{O} I(d)$, find the Hilbert function of the local ring of the Schubert variety $X^{w}$ at the point $\mathfrak{e}(v)$.

\subsection{Basic notation}

Let an element $v$ of $\mathfrak{O} I(d)$ remain fixed. Let $\mathfrak{R}(v)$ denote the set of all ordered pairs $(r, c), 1 \leq r, c \leq 2 d$, such that $r$ is not and $c$ is an entry of $v$. The picture below shows a drawing of $\Re(v)$. We think of $r$ and $c$ in $(r, c)$ as row index and column index respectively. The columns are indexed from left to right by the entries of $v$ in ascending order, the rows from top to bottom by the entries of $\{1, \ldots, 2 d\} \backslash v$ in ascending order.

We refer to $\mathfrak{d}(v)$ as the diagonal. The points of $\mathfrak{O} \Re(v)$ are those that are (strictly) above the diagonal, and the points of $\mathfrak{N}(v)$ are those that are to the South-West of the poly-line captioned 'boundary of $\mathfrak{N}(v)$ ': we draw the boundary so that points on the boundary belong to $\mathfrak{N}(v)$. The reader can readily verify that $d=13$ and $v=(1,2,3,4,6,7,10,11,13,15,18,19,22)$ for the particular picture drawn. The points of $\mathfrak{O N}(v)$ indicated by solid circles form a $v$-chain $(\$ 2.4 .1)$. 


$$
\begin{aligned}
\mathfrak{N}(v) & :=\{(r, c) \in \mathfrak{R}(v) \mid r>c\} \\
\mathfrak{O} \mathfrak{R}(v) & :=\left\{(r, c) \in \mathfrak{R}(v) \mid r<c^{*}\right\} \\
\mathfrak{O N}(v) & :=\left\{(r, c) \in \mathfrak{R}(v) \mid r>c, r<c^{*}\right\} \\
& =\mathfrak{O} \mathfrak{R}(v) \cap \mathfrak{N}(v) \\
\mathfrak{d}(v) & :=\left\{(r, c) \in \mathfrak{R}(v) \mid r=c^{*}\right\}
\end{aligned}
$$

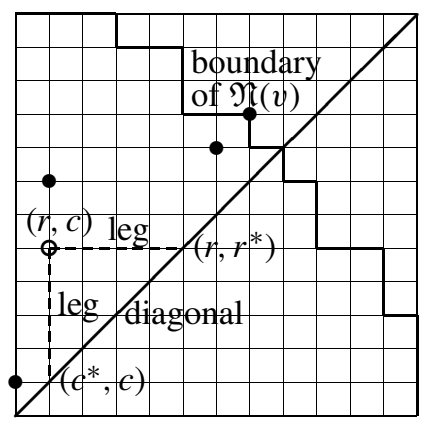

We will be considering monomials, also called multisets, in some of these sets. A monomial, as usual, is a subset with each member being allowed a multiplicity (taking values in the non-negative integers). Its degree is the sum of all multiplicities. The intersection of a monomial in a set with a subset of the set is a monomial in the subset, the multiplicities being those in the original monomial.

For $\alpha=(r, c)$ in $\mathfrak{O} \mathfrak{R}(v)$, the elements $p_{v}(\alpha):=\left(c^{*}, c\right)$ and $p_{h}(\alpha):=\left(r, r^{*}\right)$ of the diagonal $\mathfrak{d}(v)$ are respectively the vertical and horizontal projections of $\alpha$. The lines joining $\alpha$ to its projections are the legs of $\alpha$.

For $\alpha=(r, c)$ in $\mathfrak{R}(v)$, define $\alpha^{\#}:=\left(c^{*}, r^{*}\right)$. The involution $\alpha \mapsto \alpha^{\#}$ is just the reflection with respect to the diagonal $\mathfrak{d}(v)$. For a subset or even multiset $\mathfrak{E}$ of $\mathfrak{R}(v)$, the symbol $\mathfrak{E}^{\#}$ has the obvious meaning. We call $\mathfrak{E}$ symmetric if $\mathfrak{E}=\mathfrak{E}^{\#}$.

For $\alpha=(r, c)$ of $\Re(v)$, we define $\alpha$ (up) to be $\alpha$ itself if $\alpha$ is either on or above the diagonal $\mathfrak{d}(v)$ (more precisely, if $r \leq c^{*}$ ), and to be its reflection $\alpha^{\#}$ in the diagonal if $\alpha$ is below the diagonal (more precisely, if $r>c^{*}$ ). For a monomial $\mathfrak{E}$ of $\mathfrak{R}(v)$, we define $\mathfrak{E}($ up) to be the intersection of $\mathfrak{E}$ (as a multiset) with the subset $\mathfrak{O} \Re(v) \cup \mathfrak{d}(v)$ of $\mathfrak{R}(v)$. The notations $\alpha$ (down) and $\mathfrak{E}($ down) have similar meanings.

For $(r, c)$ and $(R, C)$ in $\mathfrak{R}(v)$, to write $(R, C)>(r, c)$ means that $R>r$ and $C<c$; to say $(R, C)$ dominates $(r, c)$ means that $r \leq R$ and $C \leq c$ (in terms of pictures, $(r, c)$ lies (not necessarily strictly) to the Northeast of $(R, C)$ ); to say they are comparable means that either $(R, C)>(r, c)$ or $(r, c)>(R, C)$-it is convenient to exclude equality in comparability.

For an integer $i$, we let $i$ (odd) be the largest odd integer not bigger than $i$ and $i$ (even) the smallest even integer not smaller than $i$.

\subsection{Basic definitions}

\section{$2.4 .1 v$-chain}

An ordered sequence $\alpha, \beta, \ldots$ of elements of $\mathfrak{N}(v)$ is called a $v$-chain if $\alpha>\beta>\ldots$. A $v$-chain $\alpha_{1}>\ldots>\alpha_{\ell}$ has head $\alpha_{1}$, tail $\alpha_{\ell}$, and length $\ell$.

\subsubsection{The “connection” relation on elements of a $v$-chain}

Let $C: \alpha_{1}=\left(r_{1}, c_{1}\right)>\alpha_{2}=\left(r_{2}, c_{2}\right)>\cdots$ be a $v$-chain in $\mathfrak{O N}(v)$. Two consecutive elements $\alpha_{j}$ and $\alpha_{j+1}$ of $C$ are said to be connected if the following conditions are both satisfied: 
- their legs are "intertwined"; equivalently and more precisely, this means that $r_{j}^{*} \geq$ $c_{j+1}$, or, what amounts to the same, $r_{j} \leq c_{j+1}^{*}$.

- the point $\left(r_{j+1}, r_{j}^{*}\right)$ belongs to $\mathfrak{N}(v)$; this just means that $r_{j+1}>r_{j}^{*}$.

Consider the coarsest equivalence relation on the elements of $C$ generated by the above relation. The equivalence classes of $C$ with respect to this equivalence relation are called the connected components of the $v$-chain $C$.

This definition has its quirks:

The $v$-chain $C: \alpha>\beta>\gamma$ in the picture has $\{\alpha, \beta\}$ and $\{\gamma\}$ as its connected components; but the "sub" $v$-chain $\alpha>\gamma$ of $C$ is connected (as a $v$-chain in its own right).

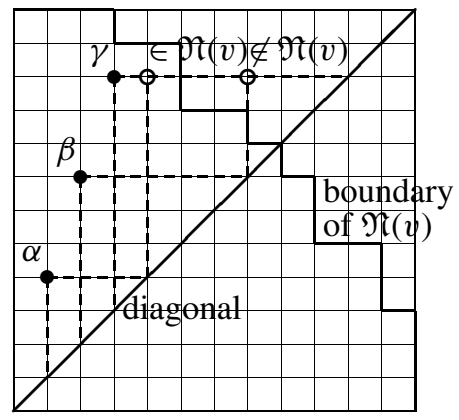

\subsubsection{The subset $\mathfrak{O m o n}_{C}$ attached to a $v$-chain $C$ in $\mathfrak{O N}(v)$}

Let $C$ be a $v$-chain in $\mathfrak{O N}(v)$. We will define $\mathfrak{O m o n}_{C}$ as a multiset of $\mathfrak{N}(v)$. It is easy to see and in any case stated explicitly as part of Corollary 5.1.5 that it is multiplicity free and so is actually a subset of $\mathfrak{N}(v)$.

First suppose that $C: \alpha_{1}=\left(r_{1}, c_{1}\right)>\cdots>\alpha_{\ell}=\left(r_{\ell}, c_{\ell}\right)$ is a connected $v$-chain in $\mathfrak{O N}(v)$. Observe that, if there is at all an integer $j, 1 \leq j \leq \ell$, such that the horizontal projection $p_{h}\left(\alpha_{j}\right)$ does not belong to $\mathfrak{N}(v)$, then $j=\ell$. Define

$$
\mathfrak{O m o n}_{C}:= \begin{cases}\left\{p_{v}\left(\alpha_{1}\right), \ldots, p_{v}\left(\alpha_{\ell}\right)\right\} & \text { if } \ell \text { is even } \\ \left\{p_{v}\left(\alpha_{1}\right), \ldots, p_{v}\left(\alpha_{\ell}\right)\right\} \cup\left\{p_{h}\left(\alpha_{\ell}\right)\right\} & \text { if } \ell \text { is odd and } p_{h}\left(\alpha_{\ell}\right) \in \mathfrak{N}(v) \\ \left\{p_{v}\left(\alpha_{1}\right), \ldots, p_{v}\left(\alpha_{\ell-1}\right)\right\} \cup\left\{\alpha_{\ell}, \alpha_{\ell}^{\#}\right\} & \text { if } \ell \text { is odd and } p_{h}\left(\alpha_{\ell}\right) \notin \mathfrak{N}(v)\end{cases}
$$

For a $v$-chain $C$ that is not necessarily connected, let $C=C_{1} \cup C_{2} \cup \cdots$ be the partition of $C$ into its connected components, and set

$$
\mathfrak{O m o n}_{C}:=\mathfrak{O m o n}_{C_{1}} \cup \mathfrak{O m o n}_{C_{2}} \cup \cdots
$$

Example 2.4.1 Let us now illustrate the definition of $\mathfrak{O m o n}_{C}$ for the $v$-chain $C:=\alpha_{1}>\cdots>\alpha_{6}$ in Figure 1. The connected components of $C$ are $C_{1}:=\alpha_{1}>$ $\alpha_{2}, C_{2}:=\alpha_{3}>\alpha_{4}>\alpha_{5}$, and $C_{3}:=\alpha_{6}$. We have $\mathfrak{O m o n}_{C_{1}}=\left\{p_{v}\left(\alpha_{1}\right), p_{v}\left(\alpha_{2}\right)\right\}$, $\mathfrak{O m o n}_{C_{2}}=\left\{p_{v}\left(\alpha_{3}\right), p_{v}\left(\alpha_{4}\right), p_{v}\left(\alpha_{5}\right), p_{h}\left(\alpha_{5}\right)\right\}, \mathfrak{O m o n}_{C_{3}}=\left\{\alpha_{6}, \alpha_{6}^{\#}\right\}$, and $\mathfrak{O m o n}_{C}=$ $\mathfrak{O m o n}_{C_{1}} \cup \mathfrak{O m o n}_{C_{2}} \cup \mathfrak{O m o n}_{C_{3}}$. It is worthwhile to note that the sub- $v$-chain $C_{4}:=$ $\alpha_{3}>\alpha_{4}>\alpha_{6}$ of $C$ is connected (the point $A$ in the figure belongs to $\mathfrak{N}(v)$ ); $\mathfrak{O m o n}_{C_{4}}=\left\{p_{v}\left(\alpha_{3}\right), p_{v}\left(\alpha_{4}\right)\right\} \cup\left\{\alpha_{6}, \alpha_{6}^{\#}\right\}$. 
Fig. 1 A $v$-chain $C$

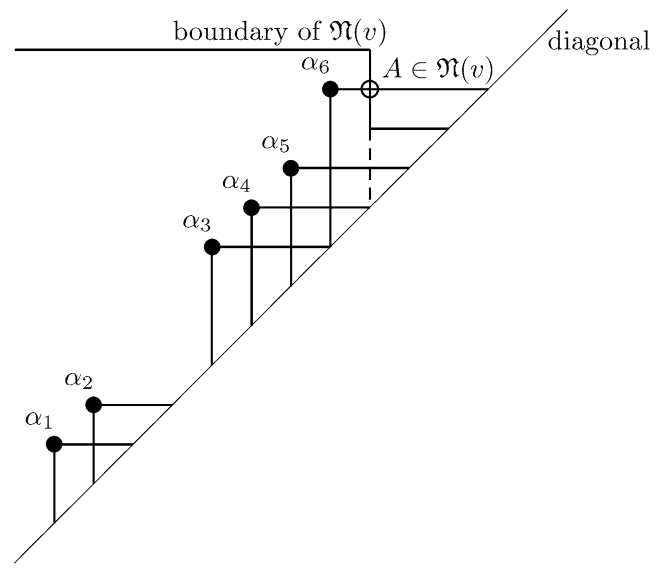

Remark 2.4.2 The definition of $\mathfrak{O m o n} \mathfrak{m}_{C}$ is admittedly ad hoc. One could try to translate it to a less specific context using general concepts about Weyl groups. We have chosen not to take such an approach because we do not know how to carry out the proof in a more general context.

We now try to motivate the definition of $\mathfrak{O m o n} \mathfrak{m}_{C}$ in $\$ 2.4 .3$ above. Our strategy of proof requires a good notion of 'domination' on monomials in $\mathfrak{O} \Re(v)$ : there should correspond to each monomial an element of $\mathfrak{O} I(d)$ that is the least among those that 'dominate' it. The notion in [7] of domination is just not good enough here, so we are forced to modify it. In $\S 2.4 .4$ and $\$ 2.6 .5$ below, we give two equivalent definitions of the new notion of domination, called $\mathfrak{O}$-domination, for $v$-chains in $\mathfrak{O N}(v)$. Both of these use the association $C \mapsto \mathfrak{O m o n}_{C}$ just defined.

The reason why the notion of domination in [7] does not work here can be seen from Proposition 2.6.1 below. For the dominating element to belong to $\mathfrak{O} I(d)$, the monomial $\mathfrak{O m o n}_{C}$ must not only be 'distinguished' in the sense of [7] (the notion is recalled in $\$ 2.6 .1$ below) but also be symmetric about the diagonal and contain evenly many elements of the diagonal. Given a connected $v$-chain $C=\alpha_{1}>\ldots>\alpha_{l}$, the only things we can say for sure about all its projections on the diagonal are: $p_{v}\left(\alpha_{1}\right)>p_{v}\left(\alpha_{2}\right)>\cdots>p_{v}\left(\alpha_{l}\right)>p_{h}\left(\alpha_{l}\right)$ and $p_{v}\left(\alpha_{1}\right)>p_{h}\left(\alpha_{1}\right)>p_{h}\left(\alpha_{2}\right)>\cdots>$ $p_{h}\left(\alpha_{l}\right)$. This explains somewhat the occurrence of the first sequence in the right hand side of the definition in $\$ 2.4 .3$.

\subsubsection{A first definition of $\mathfrak{O}$-domination}

An element $w$ of $\mathfrak{O} I(d)$ is said to $\mathfrak{D}$-dominate a $v$-chain $C$ if $w$ dominates in the sense of [7] the monomial $\mathfrak{O m o n}_{C}$ : an equivalent definition is given in $§ 2.6 .5$ below. An element $w$ of $\mathfrak{O} I(d) \mathfrak{D}$-dominates a monomial $\mathfrak{E}$ of $\mathfrak{O N}(v)$ (respectively of $\mathfrak{O} \mathfrak{R}(v)$ ) if it $\mathfrak{O}$-dominates every $v$-chain in $\mathfrak{E}$ (respectively in $\mathfrak{E} \cap \mathfrak{O N}(v)$ ).

2.5 The main theorem and its corollary

Theorem 2.5.1 Let $\mathfrak{O} R^{w}(v)$ denote the associated graded ring with respect to the unique maximal ideal of the local ring of germs at $\mathfrak{e}(v)$ of functions on $X^{w}$. Then, 
for any non-negative integer $m$, the dimension as a vector space of the homogeneous piece of $\mathfrak{O} R^{w}(v)$ of degree $m$ equals the cardinality of the set $\mathfrak{O} S^{w}(v)(m)$ of monomials of degree $m$ of $\mathfrak{O} \mathfrak{R}(v)$ that are $\mathfrak{O}$-dominated by $w$.

The proof of this theorem occupies us for most of this paper. It is reduced in $\S 4$, by an application of standard monomial theory, to combinatorics. The resulting combinatorial problem is solved in $\$ 5-9$. For now, let us note the following immediate consequence (the proof of Corollary 2.2 of [7] holds verbatim here too):

Corollary 2.5.2 The multiplicity at the point $\mathfrak{e}(v)$ of the Schubert variety $X^{w}$ equals the number of monomials in $\mathfrak{O N}(v)$ of maximal cardinality that are square-free and $\mathfrak{O}$-dominated by $w$.

\subsection{Another definition of $\mathfrak{O}$-domination}

Our goal in this subsection is to give an equivalent definition of $\mathfrak{O}$-domination of a $v$-chain—see $\$ 2.6 .5$. In the process we recall some relevant material from [7].

\subsubsection{Distinguished subsets of $\mathfrak{N}(v)$}

Following [7, §4], we define a multiset $\mathfrak{E}$ of $\mathfrak{N}(v)$ to be distinguished, if, first of all, it is a subset in the usual sense (in other words, it is "multiplicity free"), and if, for any two distinct elements $(R, C)$ and $(r, c)$ of $\mathfrak{E}$, the following conditions are satisfied:

A. $R \neq r$ and $C \neq c$.

B. If $R>r$, then either $r<C$ or $C<c$.

In terms of pictures, condition A says that $(r, c)$ cannot lie exactly due North or East of $(R, C)$ (or the other way around); so we can assume, interchanging the two points if necessary, that $(r, c)$ lies strictly to the Northeast or Northwest of $(R, C)$; condition B now says that, if $(r, c)$ lies to the Northwest of $(R, C)$, then the point that is simultaneously due North of $(R, C)$ and due East of $(r, c)$ (namely $(r, C))$ does not belong to $\mathfrak{N}(v)$.

\subsubsection{Attaching elements $w \geq v$ of $I(d, 2 d)$ to distinguished subsets of $\mathfrak{N}(v)$}

Given a distinguished subset $\mathfrak{S}$ of $\mathfrak{N}(v)$, we can get an element $w(\geq v)$ of $I(d, 2 d)$ as follows: start with $v$, remove all its members that are column indices of elements of $\mathfrak{S}$, and add row indices of all elements of $\mathfrak{S}$. As observed in [7, Proposition 4.3], this association is a bijection (between distinguished subsets of $\mathfrak{N}(v)$ on the one hand and elements $w$ of $I(d, 2 d)$ such that $w \geq v$ on the other). We denote it by $w \leftrightarrow \mathfrak{S}_{w}$.

\subsubsection{The involution \#}

There are two natural order reversing involutions on the poset $I(d, 2 d)$ :

- $w \mapsto w^{*}$, where $w^{*}:=\left\{j^{*} \mid j \in w\right\}$;

- $w \mapsto\{1, \ldots, 2 d\} \backslash w$. 
The composition of these two commuting involutions is order preserving. We denote it by $w \mapsto w^{\#}$. The elements of $\mathfrak{O} I(d)$ are fixed under this involution.

Proposition 2.6.1 An element $w \geq v$ of $I(d, 2 d)$ belongs to $\mathfrak{O} I(d)$ if and only if the distinguished subset $\mathfrak{S}_{w}$ of $\mathfrak{N}(v)$ corresponding to it as described in $\$ 2.6 .2$ is symmetric and has evenly many diagonal elements.

Proof That the symmetry of $\mathfrak{S}_{w}$ is equivalent to the condition that $w=w^{\#}$ is proved in [4, Proposition 5.7]. Now suppose that $\mathfrak{S}_{w}$ is symmetric. We claim that for an element $(r, c)$ of $\mathfrak{S}_{w}$ that is not on the diagonal, either both $r$ and $c$ are bigger than $d$ or both are less than $d+1$. Since $\mathfrak{S}_{w}$ is symmetric, it follows that $\left(c^{*}, r^{*}\right)$ also belongs to $\mathfrak{S}_{w}$. Since $\mathfrak{S}_{w}$ is distinguished, it follows that in case $r<c^{*}$ (that is, if $(r, c)$ lies above the diagonal), we have $r<r^{*}$, and so $c<r<r^{*}$; and in case $r>c^{*}$, we have $c^{*}<c$, and so $c^{*}<c<r$, and the claim is proved. The result follows easily from the claim.

\subsubsection{The element $\mathfrak{O} w_{C}$ of $\mathfrak{O} I(d)$ attached to a $v$-chain $C$}

It is obvious from its definition $(\$ 2.4 .3)$ that $\mathfrak{D m o n}_{C}$ is symmetric in the sense of $\S 2.6 .3$ and has evenly many elements on the diagonal. It is also distinguished in the sense of $\$ 2.6 .1$ (Corollary 5.1.5). We denote by $\mathfrak{O} w_{C}$ the element of $I(d, 2 d)$ associated to $\mathfrak{O} \mathfrak{m o n}{ }_{C}$ (as in $§ 2.4 .3$ ). It belongs to $\mathfrak{O} I(d)$ (Proposition 2.6.1).

\subsubsection{A second definition of $\mathfrak{O}$-domination}

From [7, Lemma 5.5] it follows that an element $w$ of $\mathfrak{O} I(d) \mathfrak{O}$-dominates a $v$-chain $C$ in $\mathfrak{O N}(v)$ if and only if $w \geq \mathfrak{O} w_{C}$, where $\mathfrak{O} w_{C}$ is as defined in $\S 2.6 .4$.

\section{Multiplicity counts certain non-intersecting lattice paths}

Fix notation as in Theorem 2.5.1. The monomials in Corollary 2.5.2 can be interpreted as certain non-intersecting lattice paths. It follows that the multiplicity equals the number of these paths. This interpretation generalizes those in $[3,5]$. We first illustrate by means of examples and then provide a brief justification.

\subsection{Description and illustration}

The grid in Figure 2 represents points of $\mathfrak{O N}(v)$ and those of the diagonal in $\mathfrak{N}(v)$ for $v, w$ as in the following example.

Example 3.1.1 Let $d=23$,

$$
\begin{aligned}
& v=(1,2,3,4,5,11,12,13,14,19,20,22,23,26,29,30,31,32,37,38,39,40,41), \\
& w=(4,5,9,10,14,17,18,21,23,25,27,28,31,32,34,35,36,39,40,41,44,45
\end{aligned}
$$
46), 
$\beta($ start $)=(6,3)$ and

$\beta$ (finish) $=(9,5)$ for $\beta=(9,3)$;

$\beta$ (start $)=\beta$ (finish $)=(21,20)$ for $\beta=(21,20)$;

$\beta($ start $)=(15,11)$ for the diagonal element $\beta=(36,11)$;

$\beta$ (start $)=(6,1)$ for the diagonal element $\beta=(46,1)$.

In the particular case (of non-intersecting lattice paths) shown,

$\beta($ finish $)=(27,19)$ for $\beta=(36,11)$,

$\beta$ (finish) $=(28,14)$ for $\beta=(46,1)$.

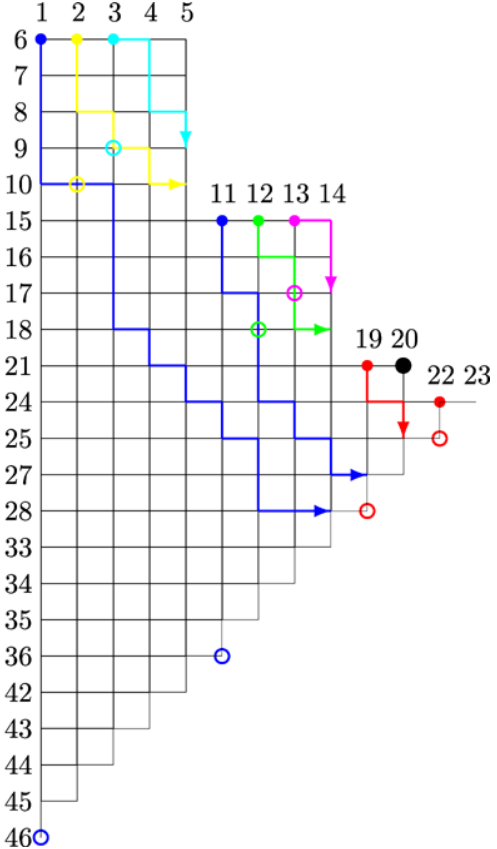

Fig. 2 An element of $\mathfrak{O P a t h s}^{w}(v)$ with $v$ and $w$ as in Example 3.1.1

so that

$$
\begin{aligned}
\mathfrak{S}_{w}=\{(9,3),(10,2),(17,13),(18,12),(21,20),(25,22),(27,26), \\
(28,19),(34,30),(35,29),(36,11),(44,38),(45,37),(46,1)\},
\end{aligned}
$$

$\mathfrak{S}_{w}(\operatorname{up})=$

$$
\{(9,3),(10,2),(17,13),(18,12),(21,20),(25,22),(28,19),(36,11),(46,1)\} .
$$

In Figure 2, The open circles represent the points of $\mathfrak{S}_{w}$ (up). From each point $\beta$ of $\mathfrak{S}_{w}$ (up) we draw a vertical line upwards from $\beta$ and let $\beta$ (start) denote the top most point of $\mathfrak{O N}(v)$ on this line. In case $\beta$ is not on the diagonal, draw also a horizontal line rightwards from $\beta$ and let $\beta$ (finish) denote the right most point of $\mathfrak{D N}(v)$ on this line. In case $\beta$ is on the diagonal, then $\beta$ (finish) is not a fixed point but varies subject to the following constraints:

- $\beta$ (finish) is one step away from the diagonal (that is, it is of the form $(r, c)$, for some entry $c$ of $v$, where $r$ is the largest integer less than $c^{*}$ that is not an entry of $v$ );

- the column index of $\beta$ (finish) is not less than that of $\beta$;

- if $\operatorname{depth}_{\mathfrak{S}_{w}} \beta$ (that is, the largest integer $\ell$ such that there is a sequence $\alpha_{1}>\ldots>$ $\alpha_{\ell}=\beta$ in $\mathfrak{S}_{w}$ ) is odd, then the horizontal projection of $\beta$ (finish) is the same as the vertical projection of $\gamma$ (finish) where $\gamma$ is the diagonal element of $\mathfrak{S}_{w}$ of depth 1 more than that of $\beta$. 


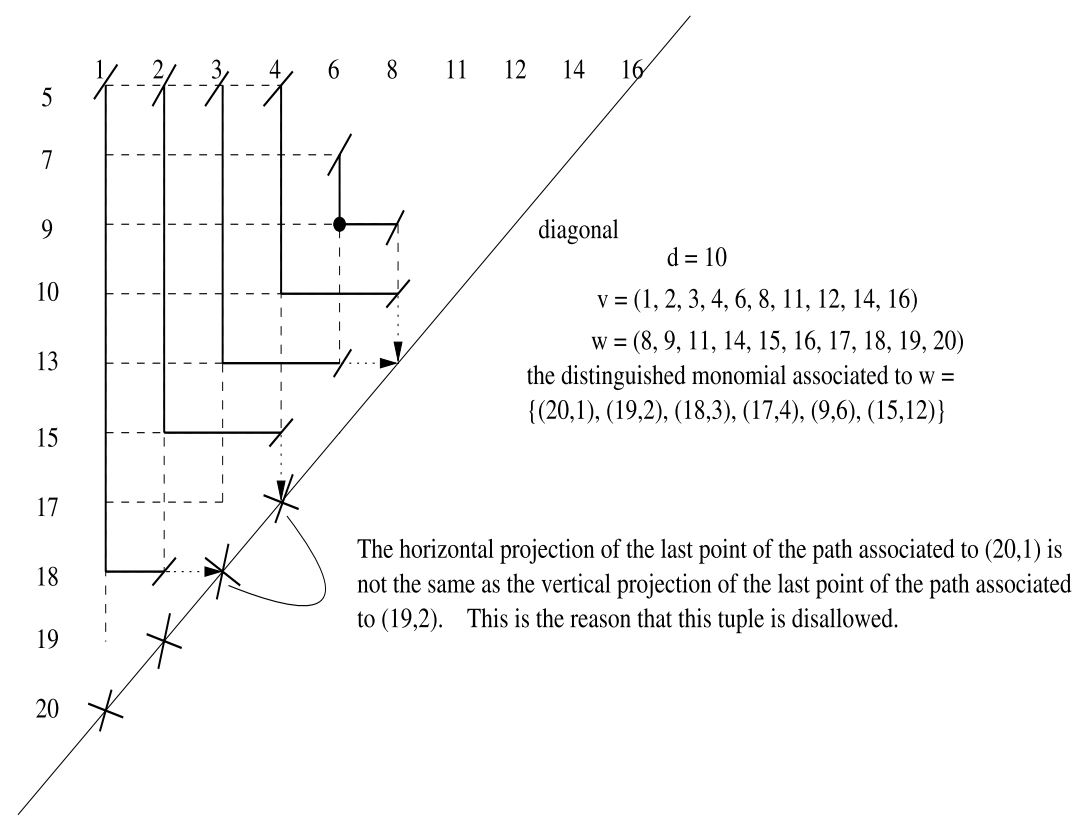

Fig. 3 A disallowed tuple of lattice paths

A lattice path between a pair of such points $\beta$ (start) and $\beta$ (finish) is a sequence $\alpha_{1}, \ldots, \alpha_{q}$ of elements of $\mathfrak{D N}(v)$ with $\alpha_{1}=\beta$ (start) and $\alpha_{q}=\beta$ (finish) such that, for $1 \leq j \leq q-1$, if we let $\alpha_{j}=(r, c)$, then $\alpha_{j+1}$ is either $(R, c)$ or $(r, C)$ where $R$ is the least element of $\{1, \ldots, 2 d\} \backslash v$ that is bigger than $r$ and $C$ the least element of $v$ that is bigger than $c$.

Consider the set $\mathfrak{O P a t h \mathfrak { s } ^ { w }}(v)$ of all tuples $\left(\Lambda_{\beta}\right)_{\beta \in \mathfrak{S}_{w} \text { (up) }}$ of paths where

- $\Lambda_{\beta}$ is a lattice path between $\beta$ (start) and $\beta$ (finish) (if $\beta$ is on the diagonal, then $\beta$ (finish) is allowed to vary in the manner described above);

- $\Lambda_{\beta}$ and $\Lambda_{\gamma}$ do not intersect for $\beta \neq \gamma$.

The number of such $p$-tuples, where $p:=\mid \mathfrak{S}_{w}($ up $) \mid$, is the multiplicity of $X^{w}$ at the point $\mathfrak{e}(v)$. A particular element of $\mathfrak{O P a t h} \mathfrak{s}^{w}(v)$ is depicted in Figure 2 .

Example 3.1.2 The reader is invited to verify that the multiplicity is 15 in the following simple case: $d=7, v=(1,2,3,4,7,9,10), w=(4,6,7,10,12,13,14)$.

Example 3.1.3 Figure 3 shows a tuple of paths not in $\mathfrak{O P a t h s}^{w}(v)$.

\subsection{Justification for the interpretation}

Any monomial as in Corollary 2.5.2 contains $\mathfrak{O} \mathfrak{R}(v) \backslash \mathfrak{O N}(v)$, for, by the definition of $\mathfrak{O}$-domination, adding or removing elements of $\mathfrak{O} \mathfrak{R}(v) \backslash \mathfrak{O N}(v)$ to or from a monomial does not alter the status of its $\mathfrak{O}$-domination. One could therefore equally 
well consider the set $\mathfrak{O} \mathcal{M}^{w}(v)$ of monomials in $\mathfrak{O N}(v)$ of maximal cardinality that are square-free and $\mathfrak{O}$-dominated by $w$. Any tuple of paths in $\mathfrak{O P a t h} \mathfrak{s}^{w}(v)$ can be considered as a square-free monomial in $\mathfrak{O N}(v)$ in the obvious way. This provides a bijection between $\mathfrak{O P a t h} \mathfrak{s}^{w}(v)$ and $\mathfrak{O M}^{w}(v)$, similar to its counterparts in the Grassmannian and symplectic Grassmannian cases in [4, 7]. For a detailed formal proof of the bijection, see $[20, \S 11.2]$.

\section{Reduction to combinatorics}

In this section we reduce the proof of our main Theorem 2.5.1 to those of some combinatorial statements.

\subsection{The tangent space to $\mathfrak{M}_{d}(V)$ at $\mathfrak{e}(v)$}

Fix notation as in $\S 2.1-\S 2.3$. Let $\mathfrak{M}_{d}(V) \subseteq G_{d}(V) \hookrightarrow \mathbb{P}\left(\wedge^{d} V\right)$ be the Plücker embedding (where $G_{d}(V)$ denotes the Grassmannian of all $d$-dimensional subspaces of $V)$. For $\theta$ in $I(d, 2 d)$, let $p_{\theta}$ denote the corresponding Plücker coordinate. Consider the affine patch $\mathbb{A}(v)$ of $\mathbb{P}\left(\wedge^{d} V\right)$ given by $p_{v} \neq 0$, where $v$ is some fixed element of $\mathfrak{O} I(d)(\subseteq I(d, 2 d))$. The affine patch $\mathfrak{O} \mathbb{A}(v):=\mathfrak{M}_{d}(V) \cap \mathbb{A}(v)$ of the orthogonal Grassmannian $\mathfrak{M}_{d}(V)$ is an affine space whose coordinate ring can be taken to be the polynomial ring in variables of the form $X_{(r, c)}$ with $(r, c) \in \mathfrak{O} \Re(v)$. Taking $d=5$ and $v=(1,3,4,6,9)$ for example, a general element of $\mathfrak{O A}(v)$ has a basis consisting of column vectors of a matrix of the following form:

$$
\left(\begin{array}{ccccc}
1 & 0 & 0 & 0 & 0 \\
X_{21} & X_{23} & X_{24} & X_{26} & 0 \\
0 & 1 & 0 & 0 & 0 \\
0 & 0 & 1 & 0 & 0 \\
X_{51} & X_{53} & X_{54} & 0 & -X_{26} \\
0 & 0 & 0 & 1 & 0 \\
X_{71} & X_{73} & 0 & -X_{54} & -X_{24} \\
X_{81} & 0 & -X_{73} & -X_{53} & -X_{23} \\
0 & 0 & 0 & 0 & 1 \\
0 & -X_{81} & -X_{71} & -X_{51} & -X_{21}
\end{array}\right)
$$

The origin of the affine space $\mathfrak{O A}(v)$, namely the point at which all $X_{(r, c)}$ vanish, corresponds clearly to $\mathfrak{e}(v)$. The tangent space to $\mathfrak{M}_{d}(V)$ at $\mathfrak{e}(v)$ can therefore be identified with the affine space $\mathfrak{O A}(v)$ with co-ordinate functions $X_{(r, c)},(r, c) \in \mathfrak{O R}(v)$.

4.2 The ideal $I$ of the tangent cone to $X^{w}$ at $\mathfrak{e}(v)$

Fix elements $v \leq w$ of $\mathfrak{O} I(d)$. Set $Y^{w}(v):=X^{w} \cap \mathfrak{O} \mathbb{A}(v)$, where $X^{w}$ is the Schubert variety indexed by $w$ and $\mathfrak{O A}(v)$ is the affine patch around $\mathfrak{e}(v)$ as in $\S 4.1$. From [13, 19] we can deduce a set of generators for the ideal $I$ of functions on $\mathfrak{O A}(v)$ vanishing on $Y^{w}(v)$ (see for example [20,§3.2.2]). We recall this result now. 
In the matrix (4.1.1), columns are numbered by the entries of $v$, the rows by $1, \ldots$, $2 d$. For $\theta \in \mathfrak{O} I(d)$, consider the submatrix given by the rows numbered $\theta \backslash v$ and columns numbered $v \backslash \theta$. Such a submatrix being of even size and skew-symmetric along the anti-diagonal, we can define its Pfaffian (a square root of the determinant of the submatrix, defined up to sign; see $[18, \S 4]$ ). Let $f_{\theta}$ denote this Pfaffian. We have

$$
I=\left(f_{\tau} \mid \tau \in \mathfrak{O} I(d), \tau \not \leq w\right) .
$$

We are interested in the tangent cone to $X^{w}$ at $\mathfrak{e}(v)$ or, what is the same, the tangent cone to $Y^{w}(v) \subseteq \mathfrak{O} \mathbb{A}(v)$ at the origin. Observe that $f_{\theta}$ is a homogeneous polynomial of degree $\mathfrak{O}-v$-degree $(\theta)$, where the $\mathfrak{O}$ - $v$-degree $(\theta)$ is defined as one half of the cardinality of $v \backslash \theta$. Because of this, $Y^{w}(v)$ itself is a cone and so equal to its tangent cone. The ideal of the tangent cone is therefore the ideal $I$ in (4.2.1).

The coordinate ring $\mathfrak{k}\left[Y^{w}(v)\right]$ of $Y^{w}(v)$ is a quotient of the polynomial ring $\mathfrak{E}[\mathfrak{O A}(v)]$, and the proposition that follows identifies a subset of the monomials in $f_{\theta}$ which forms a $\mathfrak{k}$-basis for $\mathfrak{k}\left[Y^{w}(v)\right]$. A totally ordered sequence $\theta_{1} \geq \ldots \geq \theta_{t}$ of elements in $\mathfrak{O} I(d)$ is called a standard monomial (in $\mathfrak{O} I(d)$ ). Such a monomial is $v$-compatible if for each $k, 1 \leq k \leq t$, either $\theta_{k} \gtrless v$ or $v \geq \theta_{k}$; it is $w$-dominated, for an element $w$ of $\mathfrak{O} I(d)$, if $w \geq \theta_{1}$. We denote by $\mathfrak{D S} M^{w}(v)$ the set of $w$-dominated $v$-compatible standard monomials.

Proposition 4.2.1 As $\theta_{1} \geq \ldots \geq \theta_{t}$ runs over the set $\mathfrak{O S M}^{w}(v)$ of $w$-dominated $v$-compatible standard monomials, the elements $f_{\theta_{1}} \cdots f_{\theta_{t}}$ form a basis for the coordinate ring $\mathfrak{k}\left[Y^{w}(v)\right]$ of the affine patch $Y^{w}(v)=X^{w} \cap \mathfrak{O} \mathbb{A}(v)$ of the Schubert variety $X^{w}$.

Proof This follows from results of [13, 19]. See [15] or [20, Proposition 3.2.1].

Proposition 4.2.1 says that the graded piece of $\mathfrak{k}\left[Y^{w}(v)\right]$ of degree $m$ is generated as a $\mathfrak{k}$-vector space by elements of degree $m$ of the set $\mathfrak{D} S M^{w}(v)$ of $w$-dominated $v$ compatible standard monomials, where the degree of a standard monomial $\theta_{1} \geq \ldots \geq$ $\theta_{t}$ is defined to be the sum of the $\mathfrak{O}$ - $v$-degrees of $\theta_{1}, \ldots, \theta_{t}$. To prove Theorem 2.5.1 it therefore suffices to prove the following:

Theorem 4.2.2 The set $\mathfrak{D S M}^{w}(v)(m)$ of $w$-dominated $v$-compatible standard monomials in $\mathfrak{O} I(d)$ of degree $m$ is in bijection with the set $\mathfrak{O} S^{w}(v)(m)$ of monomials in $\mathfrak{O} \mathfrak{R}(v)$ of degree $m$ that are $\mathfrak{O}$-dominated by $w$.

The proof of Theorem 4.2.2 will be reduced in $\S 4.4$ to those of Propositions 4.3.1, 4.3.2 and 4.3.3 below. These propositions will eventually be proved in $\S 9$.

\subsubsection{Illustration by an example}

We illustrate the bijection in Theorem 4.2 .2 by an example. Take $d=5$ and $v=$ $(1,2,3,6,7)$ : the elements of $\mathfrak{O} \mathfrak{R}(v)$ are depicted in Figure 4. As we will see presently in $\$ 4.3,4.4$, it is enough to restrict attention to monomials in $\mathfrak{O N}(v)$ (rather than $\mathfrak{O} \mathfrak{R}(v)$ ) and, correspondingly, standard monomials where are all elements are 


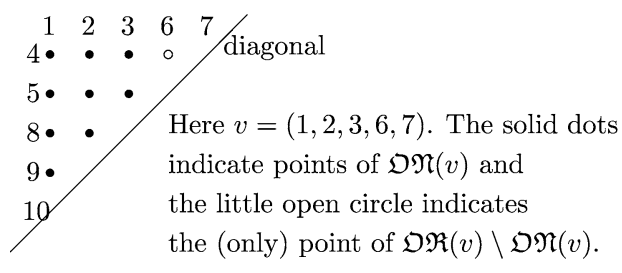

\begin{tabular}{|c|c|}
\hline \hline monomial & standard monomial \\
\hline \hline empty & empty \\
\hline$(4,3)$ & $(1,2,4,6,8)$ \\
\hline$(5,3)$ & $(1,2,5,7,8)$ \\
\hline$(4,2)$ & $(1,3,4,6,9)$ \\
\hline$(5,2)$ & $(1,3,5,7,9)$ \\
\hline$(4,3)(5,2)$ & $(1,4,5,8,9)$ \\
\hline$(4,1)$ & $(2,3,4,6,10)$ \\
\hline$(5,1)$ & $(2,3,5,7,10)$ \\
\hline$(4,3)(5,1)$ & $(2,4,5,8,10)$ \\
\hline$(4,2)(5,1)$ & $(3,4,5,9,10)$ \\
\hline$(8,2)$ & $(1,6,7,8,9)$ \\
\hline$(8,1)$ & $(2,6,7,8,10)$ \\
\hline$(8,1)$ & $(2,6,7,8,10)$ \\
\hline$(9,1)(4,3)$ & $(4,6,8,9,10)$ \\
\hline$(9,1)(5,3)$ & $(5,7,8,9,10)$ \\
\hline
\end{tabular}

\begin{tabular}{|c|c|}
\hline \hline monomial & standard monomial \\
\hline \hline$(4,3)(4,3)$ & $(1,2,4,6,8) \geq(1,2,4,6,8)$ \\
\hline$(5,3)(5,3)$ & $(1,2,5,7,8) \geq(1,2,5,7,8)$ \\
\hline$(5,3)(4,3)$ & $(1,2,5,7,8) \geq(1,2,4,6,8)$ \\
\hline$(4,2)(4,2)$ & $(1,3,4,6,9) \geq(1,3,4,6,9)$ \\
\hline$(4,2)(4,3)$ & $(1,3,4,6,9) \geq(1,2,4,6,8)$ \\
\hline$(5,2)(5,2)$ & $(1,3,5,7,9) \geq(1,3,5,7,9)$ \\
\hline$(5,2)(4,2)$ & $(1,3,5,7,9) \geq(1,3,4,6,9)$ \\
\hline$(5,2)(5,3)$ & $(1,3,5,7,9) \geq(1,2,5,7,8)$ \\
\hline$(5,3)(4,2)$ & $(1,3,5,7,9) \geq(1,2,4,6,8)$ \\
\hline$(4,1)(4,1)$ & $(2,3,4,6,10) \geq(2,3,4,6,10)$ \\
\hline$(4,1)(4,2)$ & $(2,3,4,6,10) \geq(1,3,4,6,9)$ \\
\hline$(4,1)(4,3)$ & $(2,3,4,6,10) \geq(1,2,4,6,8)$ \\
\hline$(5,1)(5,1)$ & $(2,3,5,7,10) \geq(2,3,5,7,10)$ \\
\hline$(5,1)(4,1)$ & $(2,3,5,7,10) \geq(2,3,4,6,10)$ \\
\hline$(5,1)(5,2)$ & $(2,3,5,7,10) \geq(1,3,5,7,9)$ \\
\hline$(4,1)(5,2)$ & $(2,3,5,7,10) \geq(1,3,4,6,9)$ \\
\hline$(5,1)(5,3)$ & $(2,3,5,7,10) \geq(1,2,5,7,8)$ \\
\hline$(4,1)(5,3)$ & $(2,3,5,7,10) \geq(1,2,4,6,8)$ \\
\hline$(8,2)(8,2)$ & $(1,6,7,8,9) \geq(1,6,7,8,9)$ \\
\hline$(8,2)(5,2)$ & $(1,6,7,8,9) \geq(1,3,5,7,9)$ \\
\hline$(8,2)(4,2)$ & $(1,6,7,8,9) \geq(1,3,4,6,9)$ \\
\hline$(8,2)(5,3)$ & $(1,6,7,8,9) \geq(1,2,5,7,8)$ \\
\hline$(8,2)(4,3)$ & $(1,6,7,8,9) \geq(1,2,4,6,8)$ \\
\hline$(8,1)(8,1)$ & $(2,6,7,8,10) \geq(2,6,7,8,10)$ \\
\hline$(8,1)(8,2)$ & $(2,6,7,8,10) \geq(1,6,7,8,9)$ \\
\hline$(8,1)(5,1)$ & $(2,6,7,8,10) \geq(2,3,5,7,10)$ \\
\hline$(8,1)(4,1)$ & $(2,6,7,8,10) \geq(2,3,4,6,10)$ \\
\hline$(8,2)(5,1)$ & $(2,6,7,8,10) \geq(1,3,5,7,9)$ \\
\hline$(8,2)(4,1)$ & $(2,6,7,8,10) \geq(1,3,4,6,9)$ \\
\hline$(8,1)(5,3)$ & $(2,6,7,8,10) \geq(1,2,5,7,8)$ \\
\hline$(8,1)(4,3)$ & $(2,6,7,8,10) \geq(1,2,4,6,8)$ \\
\hline$(9,1)(9,1)$ & $(3,6,7,9,10) \geq(3,6,7,9,10)$ \\
\hline$(9,1)(8,1)$ & $(3,6,7,9,10) \geq(2,6,7,8,10)$ \\
\hline$(9,1)(8,2)$ & $(3,6,7,9,10) \geq(1,6,7,8,9)$ \\
\hline$(9,1)(5,1)$ & $(3,6,7,9,10) \geq(2,3,5,7,10)$ \\
\hline$(9,1)(4,1)$ & $(3,6,7,9,10) \geq(2,3,4,6,10)$ \\
\hline$(9,1)(5,2)$ & $(3,6,7,9,10) \geq(1,3,5,7,9)$ \\
\hline$(9,1)(4,2)$ & $(3,6,7,9,10) \geq(1,3,4,6,9)$ \\
\hline$(8,1)(5,2)$ & $(3,6,7,9,10) \geq(1,2,5,7,8)$ \\
\hline$(8,1)(4,2)$ & $(3,6,7,9,10) \geq(1,2,4,6,8)$ \\
\hline &
\end{tabular}

Fig. 4 For illustrating the bijection in Theorem 4.2.2: see $\$ 4.2 .1$

$>v$. There being 9 elements in $\mathfrak{O N}(v)$, there are 55 such monomials of degree at most 2. Listed in the tables of Figure 4 are these monomials and against them the corresponding standard monomials. The correspondence will be described by means of two maps- $\mathfrak{O} \pi$ and $\mathfrak{O} \phi$-these to be described in $\$ 6$ and $\$ 7$ below. The reader is invited to return repeatedly to this example and verify the details in the course of reading $\S 6$ and $\S 7$.

The reader's attention is especially drawn to the correspondences for the monomials $(5,3)(4,2),(4,1)(5,2),(4,1)(5,3),(8,2)(5,1),(8,2)(4,1),(8,1)(5,2)$, and $(8,1)(4,2)$. As can be verified from the definition of $\mathfrak{O}$-domination, the only mono- 
mials of degree at most 2 that are not $\mathfrak{O}$-dominated by $w=(3,6,7,9,10)$ are $(9,1)(4,3)$ and $(9,1)(5,3)$. Observe that $w \geq$ the first element of every standard monomial in the tables except of those corresponding to these two monomials.

\subsection{The main combinatorial propositions}

The bijection in Theorem 4.2.2 will be described by means of two maps $\mathfrak{O} \pi$ and $\mathfrak{O} \phi$ whose definitions will be given in $\S 6$ and $\S 7$ below. We state some properties of these maps.

The map $\mathfrak{O} \pi$ associates to a monomial $\mathfrak{E}$ in $\mathfrak{O N}(v)$ a pair $\left(\mathfrak{O} w_{\mathfrak{E}}, \mathfrak{O} \Pi_{\mathfrak{E}}\right)$ consisting of an element $\mathfrak{O} w_{\mathfrak{E}}$ of $\mathfrak{O} I(d)$ and a 'smaller' monomial $\mathfrak{O} \Pi_{\mathfrak{E}}$ in $\mathfrak{O N}(v)$. This map enjoys the following good properties:

\section{Proposition 4.3.1}

(1) $\mathfrak{O} w_{\mathfrak{E}} \geq v$.

(2) $\mathfrak{O}$ - $v$-degree $\left(\mathfrak{O} w_{\mathfrak{E}}\right)+\operatorname{degree}\left(\mathfrak{O} \Pi_{\mathfrak{E}}\right)=\operatorname{degree}(\mathfrak{E})$.

(3) $\mathfrak{O} w_{\mathfrak{E}} \mathfrak{O}$-dominates $\mathfrak{O} \Pi_{\mathfrak{E}}$.

(4) $\mathfrak{O} w_{\mathfrak{E}}$ is the least element of $\mathfrak{O} I(d)$ that $\mathfrak{O}$-dominates $\mathfrak{E}$.

The map $\mathfrak{O} \phi$, on the other hand, associates a monomial in $\mathfrak{O N}(v)$ to a pair $(w, \mathfrak{T})$ consisting of an element $w$ of $\mathfrak{O} I(d)$ with $w \geq v$ and a monomial $\mathfrak{T}$ in $\mathfrak{O N}(v)$ that is $\mathfrak{O}$-dominated by $w$.

Proposition 4.3.2 The maps $\mathfrak{O} \pi$ and $\mathfrak{O} \phi$ are inverses of each other.

For an integer $f, 1 \leq f \leq 2 d$, consider the following conditions, the first on a monomial $\mathfrak{E}$ in $\mathfrak{O N}(v)$, the second on an element $w$ of $\mathfrak{O} I(d)$ :

$(\ddagger) f$ is not the row index of any element of $\mathfrak{E}$ and $f^{\star}(:=2 d-f+1)$ is not

the column index of any element of $\mathfrak{E}$.

(†) $f$ is not an entry of $w$.

(It is convenient to the use the same notation ( $\$)$ for both conditions.)

Proposition 4.3.3 Assume that $v$ satisfies $(\ddagger)$-all references to $(\ddagger)$ in this proposition are with respect to a fixed $f, 1 \leq f \leq 2 d$.

(1) Let $w$ be an element of $\mathfrak{O} I(d)$ with $w \geq v$ and $\mathfrak{T}$ a monomial in $\mathfrak{O N}(v)$ that is $\mathfrak{O}$-dominated by $w$. If $w$ and $\mathfrak{T}$ both satisfy $(\$)$, then so does $\mathfrak{O} \phi(w, \mathfrak{T})$.

(2) If a monomial $\mathfrak{E}$ in $\mathfrak{O N}(v)$ satisfies $(\ddagger)$, then so do the 'components' $\mathfrak{O} w_{\mathfrak{E}}$ and $\mathfrak{O} \Pi_{\mathfrak{E}}$ of its image under $\mathfrak{O} \pi$.

\subsection{From the main propositions to the main theorem}

Let us now see how the Theorem 4.2.2 follows from the propositions of $\S 4.3$. Most of the following argument runs parallel to its counterparts in the case of the Grassmannian and symplectic Grassmannian-Propositions 4.3.1 and 4.3.2 have their 
counterparts in $[4,7]$-but, in the case that $d$ is odd, the part involving the "mirror image" requires additional work. This is where Proposition 4.3 .3 comes in.

We first set up some notation (which, thank goodness, will not be needed beyond this section). Let $\mathfrak{O} S(v) \mathfrak{O} T(v)$ and $\mathfrak{O} U(v)$ denote respectively the sets of monomials in $\mathfrak{O} \mathfrak{R}(v), \mathfrak{O N}(v)$, and $\mathfrak{O} \mathfrak{R}(v) \backslash \mathfrak{O N}(v)$. Superscripts, suffixes, and combinations thereof are used to modify the meanings of these symbols and also of sets of standard monomials in $\mathfrak{O} I(d)$ :

- superscript: an element of $\mathfrak{O} I(d)$ denoting domination (or $\mathfrak{O}$-domination):

- $\mathfrak{O} T^{w}(v)$ is the subset of $\mathfrak{O} T(v)$ consisting of those elements that are $\mathfrak{O}$-dominated by $w$;

- $\mathfrak{O S} M^{w}(v)$, which has already appeared above (in Proposition 4.2.1), is the set of $v$-compatible, $w$-dominated standard monomials in $\mathfrak{O} I(d)$.

- subscript: an element of $\mathfrak{O} I(d)$ applicable only to sets of standard monomials and denoting anti-domination: a standard monomial $\theta_{1} \geq \ldots \geq \theta_{t}$ is anti-dominated by $v$ if $\theta_{t} \geq v$.

- suffix “ $(m)$ ”: indicates degree.

For example, $\mathfrak{D} S M_{v}^{w}(v)(m)$ denotes the set of $v$-compatible standard monomials that are anti-dominated by $v$, dominated by $w$, and have degree $m$.

One more comment on notation before we proceed. Recall that a 'base element' $v$ of $\mathfrak{O} I(d)$ has been fixed and that we have been using the suffix ' $(v)$ ' to explicitly indicate the dependence upon $v$ of the constructions. For a brief while now (only until the end of this section) we need to simultaneously handle several base elements. The base element being used will be clear from the notation: for instance, $\mathfrak{D S M}\left(v^{*}\right)$ denotes the set of $v^{*}$-compatible standard monomials in $\mathfrak{O} I(d)$ ( $v^{*}$ is defined below).

Define the domination map from $\mathfrak{O} T(v)$ to $\mathfrak{O} I(d)$ by sending a monomial in $\mathfrak{O N}(v)$ to the least element that $\mathfrak{O}$-dominates it. Define the domination map from $\mathfrak{O} S M_{v}(v)$ to $\mathfrak{O} I(d)$ by sending $\theta_{1} \geq \ldots \geq \theta_{t}$ to $\theta_{1}$. Both these maps take, by definition, the value $v$ on the empty monomial.

We are now ready to see how Theorem 4.2.2 follows from the propositions of $\S 4.3$. Repeated application of $\mathfrak{O} \pi$ gives a map from $\mathfrak{O} T(v)$ to $\mathfrak{O} S M_{v}(v)$ that commutes with domination and preserves degree. Repeated application of $\mathfrak{O} \phi$ gives a map from $\mathfrak{O S} M_{v}(v)$ to $\mathfrak{O} T(v)$. These two maps being inverses of each other (Proposition 4.3.2), we have a bijection between $\mathfrak{O} S M_{v}(v)$ and $\mathfrak{O} T(v)$. In fact, since domination and degree are respected (Proposition 4.3.1), we get a bijection $\mathfrak{O S} M_{v}^{w}(v)(m) \cong \mathfrak{O} T^{w}(v)(m)$.

As explained below, the 'mirror image' of this bijection gives another bijection: $\mathfrak{O} S M^{v}(v)(m) \cong \mathfrak{O} U(v)(m)$. Putting the two bijections together, we get the desired result:

$$
\begin{aligned}
\mathfrak{O S} M^{w}(v)(m) & =\bigcup_{k=0}^{m} \mathfrak{O} S M_{v}^{w}(v)(k) \times \mathfrak{O S M}^{v}(v)(m-k) \\
& \cong \bigcup_{k=0}^{m} \mathfrak{O} T(v)^{w}(v)(k) \times \mathfrak{O} U(v)(m-k)=\mathfrak{O} S^{w}(v)(m) .
\end{aligned}
$$


It only remains to explain how to realize $\mathfrak{O} S M^{v}(v)(m) \cong \mathfrak{O} U(v)(m)$ as the 'mirror image' of $\mathfrak{O S} M_{v}(v)(m) \cong \mathfrak{O} T(v)(m)$. For an element $u$ of $\mathfrak{O} I(d)$, define $u^{*}:=\left(u_{d}^{*}, \ldots, u_{1}^{*}\right)$. In the case $d$ is even, the association $u \mapsto u^{*}$ is an order reversing involution, and the argument in [4] for the symplectic Grassmannian holds here too. In the case $d$ is odd, $u^{*}$ is not an element of $\mathfrak{O} I(d)$, and so some additional work is required.

Let us first do the case when $d$ is even. We get a bijection $\mathfrak{O S} M^{v}(v) \cong \mathfrak{O} S M_{v^{*}}\left(v^{*}\right)$ by associating to $\theta_{1} \geq \ldots \geq \theta_{t}$ the element $\theta_{t}^{*} \geq \ldots \geq \theta_{1}^{*}$. The sum of the $\mathfrak{D}$ $v$-degrees of $\theta_{1}, \ldots, \theta_{t}$ equals the sum of the $\mathfrak{O}$ - $v^{*}$-degrees of $\theta_{t}^{*}, \ldots, \theta_{1}^{*}$, and so $\mathfrak{O S} M^{v}(v)(m) \cong \mathfrak{O S} M_{v^{*}}\left(v^{*}\right)(m)$.

For an element $(r, c)$ of $\mathfrak{O N}\left(v^{*}\right)$, consider its flip $(c, r)$. Since $v$ belongs to $\mathfrak{O} I(d)$, the complement of $v^{*}$ in $\{1, \ldots, 2 d\}$ is $v$, and it follows that $(c, r)$ belongs to $\mathfrak{O} \Re(v) \backslash$ $\mathfrak{O N}(v)$. This induces a degree preserving bijection $\mathfrak{O} T\left(v^{*}\right) \cong \mathfrak{O} U(v)$. Putting this together with the bijection of the previous paragraph and the one deduced earlier in this section (using $\mathfrak{O} \pi$ and $\mathfrak{O} \phi$ ), we get what we want:

$$
\mathfrak{O S} M^{v}(v)(m) \cong \mathfrak{O} S M_{v^{*}}\left(v^{*}\right)(m) \cong \mathfrak{O} T\left(v^{*}\right)(m) \cong \mathfrak{O} U(v)(m) .
$$

Now suppose that $d$ is odd. Then the map $x \mapsto x^{*}$ does not map $\mathfrak{O} I(d)$ to $\mathfrak{O} I(d)$ but to $\mathfrak{O} I(d)^{*}$ (defined as the set consisting of those elements $u$ of $I(d, 2 d)$ such that, for each $k, 1 \leq k \leq 2 d$, exactly one of $k, k^{*}$ belongs to $u$, and the number of entries of $u$ greater than $d$ is odd). We define a map $u \mapsto \tilde{u}$ from $\mathfrak{O} I(d)^{*}$ to $\mathfrak{O} I(d+1)$ as follows: $\tilde{u}:=\left\{\tilde{u_{1}}, \ldots, \tilde{u_{d}}, d+2\right\}$ (the elements as listed are not in increasing order except in the trivial case $u=(1, \ldots, d))$, where, for an integer $e, 1 \leq e \leq 2 d$, we set

$$
\widetilde{e}:= \begin{cases}e & \text { if } 1 \leq e \leq d \\ e+2 & \text { if } d+1 \leq e \leq 2 d\end{cases}
$$

This map $u \mapsto \widetilde{u}$ is an order preserving injection.

Consider the composition $x \mapsto x^{*} \mapsto \widetilde{x^{*}}$ from $\mathfrak{O} I(d)$ to $\mathfrak{O} I(d+1)$. This is an order reversing injection. The induced map on standard monomials is an injection from $\mathfrak{O} S M^{v}(v)$ to $\mathfrak{O} S M_{v^{*}}\left(\widetilde{v^{*}}\right)$. It is readily seen that the image under this map is the subset $\mathfrak{O S} M_{\tilde{v}^{*}}\left(\widetilde{v^{*}}\right)(\ddagger)$ consisting of those standard monomials all of whose elements satisfy ( $\ddagger$ ) with $f=d+1$. We have already established (using the maps $\mathfrak{O} \pi$ and $\mathfrak{O} \phi$ ) a bijection $\mathfrak{O} S M_{\widetilde{v^{*}}}\left(\widetilde{v^{*}}\right) \cong \mathfrak{O} T\left(\widetilde{v^{*}}\right)$. It follows from Proposition 4.3.3 that under this bijection the subset $\mathfrak{O S} M_{\tilde{v}^{*}}\left(\widetilde{v^{*}}\right)(\ddagger)$ maps to $\mathfrak{O} T\left(\widetilde{v^{*}}\right)(\ddagger)$ (defined as the set of those monomials in $\mathfrak{O N}\left(\tilde{v}^{*}\right)$ satisfying ( $\left.\ddagger\right)$ with $\left.f=d+1\right)$.

Now $\mathfrak{O} T\left(\widetilde{v^{*}}\right)(\ddagger)$ is in degree preserving bijection with $\mathfrak{O} U(v)$ : every element of degree 1 of $\mathfrak{O} T\left(\widetilde{v^{*}}\right)(\ddagger)$ is uniquely of the form $(\widetilde{c}, \widetilde{r})$ for $(r, c)$ in $\mathfrak{O} \mathfrak{R}(v) \backslash \mathfrak{O N}(v)$, and the desired bijection is induced from this. Putting all of these together, we finally have

$$
\mathfrak{O} S M^{v}(v) \cong \mathfrak{O} S M_{v^{*}}\left(\widetilde{v^{*}}\right)(\ddagger) \cong \mathfrak{O} T\left(\widetilde{v^{*}}\right)(\ddagger) \cong \mathfrak{O} U(v) .
$$

Thus, in order to prove our main theorem (Theorem 2.5.1), it suffices to describe the maps $\mathfrak{O} \pi$ and $\mathfrak{O} \phi$ and to prove Propositions 4.3.1-4.3.3. 


\section{D-depth}

The next three sections prepare the way for the proof of the combinatorial propositions of $\S 4.3$, which will eventually appear in $\S 9$. The concept of $\mathfrak{O}$-depth defined in $\$ 5.2$ below plays a key role in this paper. As the name suggests, it is the orthogonal analogue of the concept of depth of [7].

The definition of $\mathfrak{O}$-depth being more involved than that of depth, such properties as in Proposition 5.2.1 (1), Proposition 5.3.4 below need to be explicitly stated and proved: unlike in the case of depth, they are not immediately obvious from the definition. In $§ 5.3$, it is observed that the $\mathfrak{O}$-depth is no smaller than the depth. Also recorded there are some observations about the relation between $\mathfrak{O}$-depths and types of elements in $v$-chains.

\subsection{Type, $\mathfrak{O m o n} \mathfrak{m}_{C, \alpha}, \mathfrak{O} q_{C, \alpha}$, and criticality}

We introduce some terminology and notation. Their usefulness may not be immediately apparent.

The type of an element $\alpha_{j}$ in a connected $v$-chain $C: \alpha_{1}>\cdots>\alpha_{\ell}$ is said to be $\mathrm{V}, \mathrm{H}$, or $\mathrm{S}$, accordingly as:

$\mathrm{V}: j \neq \ell$, or $j=\ell$ and $\ell$ is even.

$\mathrm{H}: \quad j=\ell, \ell$ is odd, and $p_{h}\left(\alpha_{\ell}\right) \in \mathfrak{N}(v)$.

$\mathrm{S}: \quad j=\ell, \ell$ is odd, and $p_{h}\left(\alpha_{\ell}\right) \notin \mathfrak{N}(v)$.

Now let $\alpha$ be an element of a (not necessarily connected) $v$-chain $C$. Its type is defined to be its type in its connected component, and the set $\mathfrak{O M o n}_{C, \alpha}$ of elements of $\mathfrak{N}(v)$ generated by $\alpha$ is defined by:

$$
\mathfrak{O m o n}_{C, \alpha}:= \begin{cases}\left\{p_{v}(\alpha)\right\} & \text { if } \alpha \text { is of type } \mathrm{V} \text { in } C ; \\ \left\{p_{v}(\alpha), p_{h}(\alpha)\right\} & \text { if } \alpha \text { is of type } \mathrm{H} \text { in } C ; \\ \left\{\alpha, \alpha^{\#}\right\} & \text { if } \alpha \text { is of type } \mathrm{S} \text { in } C ;\end{cases}
$$

Also $\mathfrak{D} q_{C, \alpha}$ is defined to be $p_{v}(\alpha)$ if $\alpha$ is of type $\mathrm{V}$ or $\mathrm{H}$ and $\alpha$ if of type S. Observe that the monomial $\mathfrak{O m o n} \mathfrak{m}_{C}$ defined in $\S 2.4 .3$ equals the union $\cup_{\alpha \in C} \mathfrak{O m o n} \mathfrak{m}_{C, \alpha}$.

If $p_{h}(\alpha) \notin \mathfrak{N}(v)$, then clearly the same is true for every succeeding element. The first such element of $C$ is called the critical element.

In the $v$-chain depicted in Figure 1, the types of the elements $\alpha_{1}, \ldots, \alpha_{6}$ are respectively $\mathrm{V}, \mathrm{V}, \mathrm{V}, \mathrm{V}, \mathrm{H}$, and $\mathrm{S}$; the critical element is $\alpha_{6}$.

\section{Proposition 5.1.1}

(1) The cardinality is odd of a connected component that has an element of type $H$ or $S$. Conversely, if the cardinality of a component is odd, then it has an element of type $H$ or $S$.

(2) An element of type $H$ or $S$ can only be the last element in its component.

(3) The critical element has type either $V$ or $S$. No element before it can be of type $S$ and every element after it is of type $S$. In particular, any element that succeeds an element of type $S$ is of type $S$. 
Proposition 5.1.2 Let $\alpha>\gamma$ be elements of a $v$-chain $C$ (we are not assuming that they are consecutive).

(1) If $\alpha>\gamma$ is connected as a $v$-chain in its own right, then $\alpha$ is connected to its successor; $\alpha$ cannot be the last element in its connected component.

(2) If $\alpha>\gamma$ is not connected as a $v$-chain in its own right and the legs of $\alpha$ and $\gamma$ intertwine, then $\gamma$ is a component by itself and has type $S$.

Proposition 5.1.3 Let $E: \alpha>\ldots>\zeta$ be a $v$-chain, $D$ and $D^{\prime}$ two $v$-chains with tail $\alpha$, and $C, C^{\prime}$ the concatenations of $D, D^{\prime}$ respectively with $E$. Then

(1) The last element in the connected component containing $\alpha$ is the same in $C$ and $C^{\prime}$ (and this is the same as in $E$ ).

(2) Denoting by $\lambda$ the element in (1), the only element among $\alpha, \ldots, \zeta$ that possibly has different types in $C$ and $C^{\prime}$ is $\lambda$.

Proposition 5.1.4 Let $\alpha$ and $\beta$ be elements of a $v$-chain C. Let us use $\alpha^{\prime}$ and $\beta^{\prime}$ respectively to denote elements of $\mathfrak{O m o n}_{C, \alpha}$ (up) and $\mathfrak{O m o n}{ }_{C, \beta}$ (up).

(1) If $\alpha>\beta$ (these elements are not necessarily consecutive in $C$ ), then, given $\beta^{\prime}$, there exists $\alpha^{\prime}$ such that $\alpha^{\prime}>\beta^{\prime}$. In fact, this is true for every choice of $\alpha^{\prime}$ except when

(*) $\quad \alpha$ is of type $\mathrm{H}$, and $p_{h}(\alpha) \ngtr \beta^{\prime}$ for some $\beta^{\prime} \in \mathfrak{D m o n}_{C, \beta}$.

In particular, $\mathfrak{O} q_{C, \alpha}>\beta^{\prime}$ and $\mathfrak{O} q_{C, \alpha}>\mathfrak{O} q_{C, \beta}$.

(2) Conversely, suppose that $\alpha^{\prime}>\beta^{\prime}$ for some choice of $\alpha^{\prime}$ and $\beta^{\prime}$. Then $\alpha \geq \beta$; if equality occurs, then $\alpha$ is of type $H, \alpha^{\prime}=p_{v}(\alpha)$ and $\beta^{\prime}=p_{h}(\alpha)$. In particular, if $\alpha^{\prime}>\mathfrak{O} q_{C, \beta}$ (or more specially $\mathfrak{O} q_{C, \alpha}>\mathfrak{O} q_{C, \beta}$ ), then $\alpha>\beta$.

(3) If (*) holds for $\alpha>\beta$ in $C$, then

(a) the critical element of $C$ is the one just after $\alpha$; in particular, $\alpha$ is uniquely determined.

(b) all elements of $C$ succeeding $\alpha$ are of type $S$; in particular, $\beta$ is of type $S$ and $\beta^{\prime}=\beta$.

(c) (*) holds for $\gamma$ in place of $\beta$ for every $\gamma$ in $C$ that succeeds $\alpha$.

Proof (1) If $\alpha$ is of type $\mathrm{V}$ or $\mathrm{H}$, we need only take $\alpha^{\prime}=p_{v}(\alpha)$, for $p_{v}(\alpha)>p_{v}(\beta)$, $p_{v}(\alpha)>p_{h}(\beta)$, and $p_{v}(\alpha)>\beta$. Now suppose that $\alpha$ is of type $\mathrm{S}$. Then $\beta$ too is of type $\mathrm{S}$ (Proposition 5.1.1 (3)), so $\beta^{\prime}$ can only be $\beta$, and the first part of (1) is proved. It follows that if $\alpha^{\prime}=p_{v}(\alpha)$ or if $\alpha$ has type $\mathrm{S}$, then $\alpha^{\prime}>\beta^{\prime}$ independent of the choice of $\alpha^{\prime}$. So if $\alpha^{\prime} \ngtr \beta^{\prime}$, then (*) holds and $\alpha^{\prime}=p_{h}(\alpha)$.

(3) Let $\lambda$ be the immediate successor of $\alpha$ in $C$. Then $\alpha$ is not connected to $\lambda$ (Proposition 5.1.1 (2)). Since $p_{h}(\alpha) \ngtr \beta^{\prime}$, it follows that $\alpha$ and $\beta$ have intertwining legs. Therefore so do $\alpha$ and $\lambda$. By Proposition 5.1.2 (2), $\lambda$ has type $\mathrm{S}$ in $C$.

Since $\alpha$ has type $H$ and $\lambda$ type $S$, it follows immediately that $\lambda$ is the critical element. This proves (a). Assertion (b) now follows from Proposition 5.1.1 (3). For (c), write $p_{h}(\alpha)=\left(a, a^{*}\right), \lambda=(R, C)$, and $\gamma=(r, c)$. Then $R<a^{*}$, for $\alpha$ and $\lambda$ have intertwining legs but are not connected. So $c<r \leq R<a^{*}$. This means $p_{h}(\alpha) \ngtr \gamma$. And $\gamma$ being of type S (by (b)), we can take $\gamma^{\prime}=\gamma$. 
(2) Suppose that $\alpha \geq \beta$. Then $\beta>\alpha$. By the second part of (1) above, $\beta$ is of type $\mathrm{H}$ and $\beta^{\prime}=p_{h}(\beta)$; by item (b) of (3), $\alpha$ is of type $\mathrm{S}$, so $\alpha^{\prime}=\alpha$. This leads to the contradiction $\beta>\alpha>p_{h}(\beta)$.

Corollary 5.1.5 $\mathfrak{O m o n}_{C}$ is a distinguished subset of $\mathfrak{N}(v)$ in the sense of $\$ 2.6 .1$.

Proof If $\alpha$ in $C$ is of type $\mathrm{V}$ or $\mathrm{S}$, then $\mathfrak{O m o n}_{C, \alpha}$ is a singleton; if it is of type $\mathrm{H}$, then $\mathfrak{O m o n}_{C, \alpha}=\left\{p_{v}(\alpha), p_{h}(\beta)\right\}$. So there can be no violation of conditions A and B of $\S 2.6 .1$ by elements of $\mathfrak{O m o n}_{C, \alpha}$.

Suppose $\alpha>\beta$. By Proposition 5.1.4 (1), we have $\alpha^{\prime}>\beta^{\prime}$ for any choice of $\alpha^{\prime} \in$ $\mathfrak{O m o n}_{C, \alpha}$ and $\beta^{\prime} \in \mathfrak{O m o n}_{C, \beta}$ except when the condition (*) holds. By (3) of the same proposition, if (*) holds, then $\beta^{\prime}=\beta$, and writing $\beta=(r, c), p_{h}(\alpha)=\left(a, a^{*}\right)$, we have $r<a$ (since $\alpha>\beta$ ) and $c<r<a^{*}$ (see proof of item 3(c) of the proposition). Thus there can be no violation of conditions A and B of $\$ 2.6 .1$.

Corollary 5.1.6 Let $\mathfrak{E}$ be a $v$-chain in $\mathfrak{O N}(v)$ and $w$ an element of $\mathfrak{O} I(d)$. If $w$ $\mathfrak{O}$-dominates $\mathfrak{E}$, then $w$ dominates in the sense of [7] the monomial $\mathfrak{E} \cup \mathfrak{E}^{\#}$ of $\mathfrak{N}(v)$.

Proof By [4, Proposition 5.15], it is enough to show that $w$ dominates $\mathfrak{E}$. Let $C$ : $\alpha_{1}>\ldots>\alpha_{t}$ be a $v$-chain in $\mathfrak{E}$. Writing $\alpha_{j}=\left(r_{j}, c_{j}\right)$ and $\mathfrak{O} q_{C, \alpha_{j}}=\left(R_{j}, C_{j}\right)$ we have $r_{j} \leq R_{j}$ and $C_{j} \leq c_{j}$. By Proposition 5.1.4 (1), $\mathfrak{O} q_{C, \alpha_{1}}>\ldots>\mathfrak{D} q_{C, \alpha_{t}}$. Since $w \mathfrak{O}$-dominates $\mathfrak{E}$, it in particular dominates $\mathfrak{O} q_{C, \alpha_{1}}>\ldots>\mathfrak{O} q_{C, \alpha_{t}}$ and so also $C$.

\subsection{Definition of $\mathfrak{O}$-depth}

Recall from [7] the notion of depth of an element $\alpha$ in a monomial $\mathfrak{F}$ in $\mathfrak{N}(v)$ : it is the largest possible length of a $v$-chain in $\mathfrak{F}$ with tail $\alpha$ and denoted depth $\mathfrak{F}_{\mathfrak{F}} \alpha$. The depth of $\mathfrak{F}$ is the maximum of the depths in it of all its elements.

The $\mathfrak{O}$-depth of an element $\alpha$ in a $v$-chain $C$ in $\mathfrak{O N}(v)$ is the depth in $\mathfrak{O m o n}_{C}$ in the sense of [7] of $\mathfrak{O} q_{C, \alpha}$ : in other words, it is the depth in $\mathfrak{O m o n} \mathfrak{m}_{C}$ of $p_{v}(\alpha)$ in case $\alpha$ is of type $\mathrm{V}$ or $\mathrm{H}$, and of $\alpha$ (equivalently of $\alpha^{\#}$ ) in case $\alpha$ is of type $\mathrm{S}$. It is denoted $\mathfrak{O}$-depth $C$ ( $\alpha)$.

In the $v$-chain $C$ depicted in Figure 1, $\mathfrak{D}$-depth ${ }_{C}\left(\alpha_{i}\right)=i$ for $1 \leq i \leq 6$; setting $C_{0}:=C \backslash\left\{\alpha_{1}\right\}$, the $\mathfrak{O}$-depths in $C_{0}$ of $\alpha_{2}, \ldots, \alpha_{6}$ are respectively $1,3,4,5$, and 6 .

The $\mathfrak{O}$-depth of an element $\alpha$ in a monomial $\mathfrak{E}$ of $\mathfrak{O N}(v)$ is the maximum, over all $v$-chains $C$ in $\mathfrak{E}$ containing $\alpha$, of the $\mathfrak{O}$-depth of $\alpha$ in $C$. It is denoted $\mathfrak{O}$-depth $\mathfrak{E}_{\mathfrak{E}}(\alpha)$. Finally, the $\mathfrak{O}$-depth of a monomial $\mathfrak{E}$ in $\mathfrak{O N}(v)$ is the maximum of the $\mathfrak{O}$-depths in $\mathfrak{E}$ of all the elements of $\mathfrak{E}$.

There is a conflict in the above definitions: Is the $\mathfrak{O}$-depth of an element of a $v$ chain $C$ the same as its depth as an element of the monomial $C$ ? In other words, could the $\mathfrak{O}$-depth of an element in a $v$-chain be exceeded by its $\mathfrak{O}$-depth in a sub-chain? The conflict is resolved by the first item of the following proposition.

\section{Proposition 5.2.1}

(1) For $v$-chains $C \subseteq D$, the $\mathfrak{O}$-depth in $C$ of an element of $C$ is no more than its $\mathfrak{O}$-depth in $D$. 
(2) If a $v$-chain $C$ is an initial segment of a $v$-chain $D$, then the $\mathfrak{O}$-depths in $C$ and $D$ of an element of $C$ are the same.

Proof (1): By an induction on the difference in the cardinalities of $D$ and $C$, we may assume that $D$ has one more element than $C$. Call this extra element $\delta$. Suppose that $\delta$ lies between successive elements $\alpha$ and $\beta$ of $C$ (the modifications needed to cover the extreme cases when it goes at the beginning or the end are being left to the reader).

The only elements of $C$ that could possibly undergo changes of type on addition of $\delta$ are $\alpha$ and the last element in the connected component of $\beta$, which let us call $\beta^{\prime}$. If there are no type changes, then $\mathfrak{O m o n}_{C} \subseteq \mathfrak{O m o n}_{D}$ and the assertion is immediate. The only type change that $\alpha$ can undergo is from $\mathrm{H}$ to $\mathrm{V}$. The type changes that $\beta^{\prime}$ can undergo are: $\mathrm{H}$ to $\mathrm{V} ; \mathrm{V}$ to $\mathrm{H} ; \mathrm{S}$ to $\mathrm{V} ; \mathrm{V}$ to $\mathrm{S}$. An easy enumeration of cases shows that only one of $\alpha$ and $\beta^{\prime}$ can undergo a type change.

We need not worry about changes from $\mathrm{V}$ to $\mathrm{H}$ for in this case $\mathfrak{O m o n} C \subseteq \mathcal{O m o n}_{D}$.

First let us suppose that $\alpha$ undergoes a change of type (from $\mathrm{H}$ to $\mathrm{V}$ ). Then $\delta$ is connected to $\alpha$. It follows from Proposition 5.1.1 (1) that $\delta$ has type $\mathrm{V}$ in $D$ : the connected component of $\alpha$ in $C$ has odd number of elements, so if $\delta$ happens to be the last element in its component in $D$, the number of elements in that component will be even. Replacing an occurrence of $p_{h}(\alpha)$ in a $v$-chain of $\mathfrak{O m o n}_{C}$ by $p_{v}(\delta)$ would result in a $v$-chain in $\mathfrak{D m o n}_{D}$ (by Proposition 5.1.4 (1)), and this case is settled.

Now suppose that $\beta^{\prime}$ undergoes a type change. Then $\delta$ is connected to $\beta$ and $\delta$ is of type $\mathrm{V}$ in $D$ (Proposition 5.1.1 (2)). Replacing by $p_{v}(\delta)$ any occurrence in a $v$-chain in $\mathfrak{O m o n}_{C}$ of $p_{v}\left(\beta^{\prime}\right), p_{h}\left(\beta^{\prime}\right), \beta^{\prime}$ accordingly as the type of $\beta^{\prime}$ in $C$ is $\mathrm{V}, \mathrm{H}$, or $\mathrm{S}$, (not necessarily in the same place but at an appropriate place) gives a $v$-chain in $\mathfrak{O m o n}_{D}$ (by Proposition 5.1.4 (1)), and we see that the $\mathfrak{O}$-depth cannot decrease.

(2): Thanks to Proposition 5.1.4 (2), contributions to $\mathfrak{O m o n}_{D}$ from elements beyond $\alpha$ for an element $\alpha$ of $C$, (in particular from those not in $C$ ) do not affect the depth in $\mathfrak{O m o n} \mathfrak{m}_{D}$ of $\mathfrak{D} q_{D, \alpha}$. Looking for the possibility of differences in types in $C$ and $D$ of elements of $C$, we see that the only element of $C$ that has possibly a different type in $D$ is its last element. And this too can change type only from $\mathrm{H}$ to $\mathrm{V}$.

Corollary 5.2.2 If $C \subseteq D$ are $v$-chains in $\mathfrak{O N}(v)$, then $\mathfrak{O} w_{C} \leq \mathfrak{O} w_{D}$ (although it is not always true that $\mathfrak{O m o n}_{C} \subseteq \mathfrak{O m o n}_{D}$ ).

Proof By [7, Lemma 5.5], it is enough to show that every $v$-chain in $\mathfrak{O m o n} \mathfrak{n}_{C}$ is dominated by $\mathfrak{O} w_{D}$. Let $\beta_{1}=\left(r_{1}, c_{1}\right)>\cdots>\beta_{t}=\left(r_{t}, c_{t}\right)$ be an arbitrary $v$-chain in $\mathfrak{O m o n}_{C}$. To show that it is dominated by $\mathfrak{O} w_{D}$, it is enough, by [7, Lemma 4.5], to show the existence of a $v$-chain $\left(R_{1}, C_{1}\right)>\cdots>\left(R_{t}, C_{t}\right)$ in $\mathfrak{O m o n}_{D}$ with $r_{j} \leq R_{j}$ and $C_{j} \leq c_{j}$ for $1 \leq j \leq t$. Such a $v$-chain exists by the proof of (1) of Proposition 5.2.1.

\section{Corollary 5.2.3}

(1) Let $\mathfrak{E}$ be a monomial in $\mathfrak{O N}(v)$ and $\alpha \in \mathfrak{E}$. Then there exists a $v$-chain $C$ in $\mathfrak{E}$ with tail $\alpha$ such that $\mathfrak{O}$-depth $\mathfrak{E}_{\mathfrak{E}}(\alpha)=\mathfrak{O}$-depth $\mathrm{d}_{C}(\alpha)$.

(2) For elements $\alpha>\gamma$ in a $v$-chain $C$ (these need not be consecutive), we have $\mathfrak{O}-\operatorname{depth}_{C}(\alpha)<\mathfrak{O}-\operatorname{depth}_{C}(\gamma)$. 
(3) $\mathfrak{O}$-depth $\operatorname{EE}_{\mathfrak{E}}(\alpha)<\mathfrak{O}-\operatorname{depth}_{\mathfrak{E}}(\gamma)$, for $\alpha>\gamma$ in a monomial $\mathfrak{E}$ in $\mathfrak{O N}(v)$.

(4) No two elements of the same $\mathfrak{O}$-depth in a monomial in $\mathfrak{O N}(v)$ are comparable.

Proof (1) follows from (2) of the Proposition above and the definition of $\mathfrak{O}$-depth.

(2) This follows from Proposition 5.1.4 (1) and the definition of $\mathfrak{O}$-depth.

(3) By (1), there exists a $v$-chain $C$ with tail $\alpha$ such that $\mathfrak{O}$-depth $\operatorname{de}_{\mathfrak{E}}(\alpha)=$ $\mathfrak{O}$-depth ${ }_{C}(\alpha)$. Concatenate $C$ with $\alpha>\gamma$ and let $D$ denote the resulting $v$-chain. By (2) of the Proposition above, $\mathfrak{O}$-depth ${ }_{C}(\alpha)=\mathfrak{O}$-depth ${ }_{D}(\alpha)$. By (2) above, $\mathfrak{O}$-depth ${ }_{D}(\alpha)<\mathfrak{O}-\operatorname{depth}_{D}(\gamma)$. And finally, $\mathfrak{O}-\operatorname{depth}_{D}(\gamma) \leq \mathfrak{O}-\operatorname{depth}_{\mathfrak{E}}(\gamma)$ by the definition of $\mathfrak{O}$-depth $\mathfrak{E}_{\mathfrak{E}}(\gamma)$.

(4) is immediate from (3).

Corollary 5.2.4 Let $\beta>\gamma$ be elements of a $v$-chain $C$ of elements of $\mathfrak{O N}(v)$. Let $E$ be a $v$-chain in $\mathfrak{O m o n} \mathfrak{m}_{C}$ with tail $\mathfrak{O} q_{C, \gamma}$ and length $\mathfrak{O}$-depth ${ }_{C}(\gamma)$. Then $\mathfrak{O} q_{C, \beta}$ occurs in $E$.

Proof It is enough to show that for $\alpha^{\prime} \neq \mathfrak{D} q_{C, \beta}$ in $E$, either $\alpha^{\prime}>\mathfrak{D} q_{C, \beta}$ or $\mathfrak{O} q_{C, \beta}>$ $\alpha^{\prime}$. Let $\alpha$ be in $C$ such that $\mathfrak{O} q_{C, \beta} \neq \alpha^{\prime} \in \mathfrak{O} \mathfrak{m o n}_{C, \alpha}$. If $\beta \geq \alpha$, then $\mathfrak{O} q_{C, \beta}>\alpha^{\prime}$ by Proposition 5.1.4 (1). If $\alpha>\beta$ and $\alpha^{\prime} \ngtr \mathfrak{O} q_{C, \beta}$, then, by (1) and (3) of the same proposition, $\alpha^{\prime} \ngtr \mathfrak{D} q_{C, \gamma}$, a contradiction.

$5.3 \mathfrak{O}$-depth and depth; $\mathfrak{O}$-depth and type

Lemma 5.3.1 The $\mathfrak{O}$-depth of an element $\alpha$ in a monomial $\mathfrak{E}$ of $\mathfrak{O N}(v)$ is no less than its depth (in the sense of [7]) in $\mathfrak{E} \cup \mathfrak{E}^{\#}$.

Proof Let $C: \alpha_{1}>\ldots>\alpha_{t}$ be a $v$-chain in $\mathfrak{E} \cup \mathfrak{E}^{\#}$ with tail $\alpha_{t}=\alpha$, where $t$ is the depth of $\alpha$ in $\mathfrak{E} \cup \mathfrak{E}^{\#}$. We then have $\alpha_{1}$ (up) $>\ldots>\alpha_{t}$ (up), so we may assume $C$ to be in $\mathfrak{E}$. By Proposition 5.1.4 (1), $\mathfrak{O} q_{C, \alpha_{1}}>\ldots>\mathfrak{O} q_{C, \alpha_{t}}$ in $\mathfrak{O m o n}$ m $_{C}$. So depth $\operatorname{EEF}^{\#}(\alpha)=t \leq \operatorname{depth}_{\mathfrak{O m o n} C}\left(\mathfrak{O} q_{C, \alpha_{t}}\right) \leq \mathfrak{O}$-depth $\mathfrak{E}_{\mathfrak{E}}(\alpha)$.

\section{Lemma 5.3.2}

(1) For consecutive elements $\alpha>\beta$ of a $v$-chain $C$,

$$
\mathfrak{O}-\operatorname{depth}_{C}(\beta)= \begin{cases}\mathfrak{O}-\operatorname{depth}_{C}(\alpha)+2 & \text { if and only if } \alpha \text { is of } \\ & \text { type H and } p_{h}(\alpha)>\beta \\ \mathfrak{O}-\operatorname{depth}_{C}(\alpha)+1 & \text { otherwise }\end{cases}
$$

(2) For an element of a $v$-chain $C$ such that either its horizontal projection belongs to $\mathfrak{N}(v)$ or it is connected to its predecessor, the parity of its $\mathfrak{O}$-depth in $C$ is the same as that of its ordinality in its connected component in $C$.

(3) The $\mathfrak{O}$-depth is odd of a type H element in a $v$-chain; it is even of a type Velement that is the last in its component.

(4) If in a $v$-chain $C$ there is an element of $\mathfrak{O}$-depth $d$, then

(a) for every odd integer $d^{\prime} \leq d$, there is in $C$ an element of $\mathfrak{O}$-depth $d^{\prime}$. 
(b) if, for an even integer $d^{\prime} \leq d$, there is no element in $C$ of $\mathfrak{O}$-depth $d^{\prime}$, then the element $\alpha$ in $C$ of $\mathfrak{O}$-depth $d^{\prime}-1$ is of type $H$, and $p_{h}(\alpha)>\beta$, where $\beta$ denotes the immediate successor of $\alpha$ in $C$.

(5) Let $C$ be a $v$-chain and $\alpha$ an element of type $H$ in $C$. Then the depth in $\mathfrak{O m o n}_{C}$ of $p_{h}(\alpha)$ equals $\mathfrak{O}$-depth ${ }_{C}(\alpha)+1$. In particular, this depth is even.

Proof (1): From items 1 and 3(a) of Proposition 5.1.4, it follows that, for $\gamma$ in $C$ with $\gamma>\alpha$, if $\gamma^{\prime} \ngtr \mathfrak{O} q_{C, \alpha}$ for some $\gamma^{\prime}$ in $\mathfrak{E}_{C, \gamma}$, then $\gamma^{\prime} \ngtr \mathfrak{O} q_{C, \beta}$. Thus $\mathfrak{O}$-depth $\operatorname{dep}_{C}(\beta)$ exceeds $\mathfrak{O}$-depth ${ }_{C}(\alpha)$ by the number of elements in $\mathfrak{O m o n} \mathfrak{m}_{C, \alpha}$ that dominate $\mathfrak{O} q_{C, \beta}$. This number is 1 if $\alpha$ is of type $\mathrm{V}$, or of type $\mathrm{S}$, or of type $\mathrm{H}$ and $p_{h}(\alpha) \ngtr \beta$; it is 2 if $\alpha$ is of type $\mathrm{H}$ and $p_{h}(\alpha)>\beta$ (note that $p_{h}(\alpha)>\beta$ if and only if $p_{h}(\alpha)>\mathfrak{O} q_{C, \beta}$ ).

(2): Let $\lambda$ be such an element. Everything preceding $\lambda$ in $C$ is of type $H$ or V (Proposition 5.1.1 (3)). Let $\lambda$ belong to the $k^{\text {th }}$ connected component, and $n_{1}, \ldots$, $n_{k}$ be respectively the cardinalities of the first, $\ldots, k^{\text {th }}$ connected components. By (1) above and item 3(b) of Proposition 5.1.4, $\mathfrak{O}-\operatorname{depth}_{C}(\lambda)$ is $n_{1}$ (even) $+\cdots+n_{k-1}$ (even) plus the ordinality of $\lambda$ in the $k^{\text {th }}$ connected component.

(3): The assertions are special cases of 2 .

(4): This follows easily from (1) and (3).

(5): By Proposition 5.1.4 (2), there is no element $\gamma$ in $\mathfrak{O m o n}_{C}$ that lies between $p_{v}(\alpha)$ and $p_{h}(\alpha)$ (meaning $p_{v}(\alpha)>\gamma>p_{h}(\alpha)$ ), so the assertion holds.

Corollary 5.3.3 For a $v$-chain $C$ in $\mathfrak{O N}(v)$, if the $\mathfrak{D}$-depths of elements in $C$ are bounded by $k$, then the depths of elements in $\mathfrak{O M o n}_{C}$ are bounded by $k(\mathrm{even})$.

Proof The depth of $\mathfrak{O} q_{C, \alpha}$ in $\mathfrak{O m o n} C$ for any $\alpha$ in $C$ is at most $k$ by hypothesis. An element of $\mathfrak{O m o n}_{C}$ that is not $\mathfrak{O} q_{C, \alpha}$ for any $\alpha$ in $C$ can only be of the form $p_{h}(\alpha)$ for some $\alpha$. By Proposition 5.1.4, $\operatorname{depth}_{\mathfrak{O m o n} C} p_{v}(\alpha)=\operatorname{depth}_{\mathfrak{O m o n} C} p_{h}(\alpha)-$ 1 , which implies depth $\mathfrak{O m o n}_{C} p_{h}(\alpha) \leq k+1$. If, moreover, $k$ is even, then by (3) of Lemma 5.3.2 depth $\mathfrak{O m o n}_{C} p_{h}(\alpha)=\operatorname{depth}_{\mathfrak{O m o n}_{C}} p_{v}(\alpha)+1 \leq(k-1)+1=k$.

Proposition 5.3.4 Given $\alpha$ in a monomial $\mathfrak{E}$ in $\mathfrak{O N}(v)$, there exists a $v$-chain $C$ in $\mathfrak{E}$ with tail $\alpha$ such that $\mathfrak{O}-\operatorname{depth}_{C}(\beta)=\mathfrak{O}-\operatorname{depth}_{\mathfrak{E}}(\beta)$ for every $\beta$ in $C$.

Proof Proceed by induction on $d:=\mathfrak{O}$-depth $\operatorname{Et}_{\mathfrak{E}}(\alpha)$. Choose a $v$-chain $D$ in $\mathfrak{E}$ with tail $\alpha$ such that $\mathfrak{O}$-depth ${ }_{D}(\alpha)=\mathfrak{O}$-depth $\operatorname{Ed}_{\mathfrak{E}}(\alpha)$ (such a $v$-chain exists by Corollary 5.2.3 (1)). Let $\alpha^{\prime}$ be the element in $D$ just before $\alpha$. It follows from item (3) of Corollary 5.2.3 and item (1) of Lemma 5.3.2 that $\mathfrak{O}-\operatorname{depth}_{\mathfrak{E}}\left(\alpha^{\prime}\right)$ (as also $\mathfrak{O}$-depth $\left.{ }_{D}\left(\alpha^{\prime}\right)\right)$ is either $d-1$ or $d-2$. By induction, there exists a $v$-chain $C^{\prime}$ with tail $\alpha^{\prime}$ that has the desired property. Let $C$ be the concatenation of $C^{\prime}$ with $\alpha^{\prime}>\alpha$.

We claim that $C$ has the desired property. The only thing to be proved is that $\mathfrak{O}$-depth ${ }_{C}(\alpha)=d$. By item (1) of Lemma 5.3.2, we have $\mathfrak{O}-\operatorname{depth}_{C}(\alpha) \geq$ $\mathfrak{O}$-depth $C_{C^{\prime}}\left(\alpha^{\prime}\right)+1$. In particular, the claim is proved in case $\mathfrak{O}$-depth $C_{C^{\prime}}\left(\alpha^{\prime}\right)$ is $d-1$, so let us assume that $\mathfrak{O}$-depth $C_{C^{\prime}}\left(\alpha^{\prime}\right)$ is $d-2$. It now follows from the same item that $\alpha^{\prime}$ has type $\mathrm{H}$ in $D$ and $p_{h}\left(\alpha^{\prime}\right)>\alpha$; it further follows that it is enough to show that $\alpha^{\prime}$ has type $\mathrm{H}$ in $C$.

Since $\alpha^{\prime}$ has type $H$ in $D$, it follows (from item (2) of Proposition 5.1.1) that $\alpha^{\prime}>\alpha$ is not connected and (from item (3) of Lemma 5.3.2) that $d-2$ is odd. Now, 
by item (3) of Lemma 5.3.2, the type in $C^{\prime}$ of $\alpha^{\prime}$ cannot be $\mathrm{V}$, so it is $\mathrm{H}$, and the claim is proved.

Corollary 5.3.5 For a monomial $\mathfrak{E}$ in $\mathfrak{O N}(v), \beta$ in $\mathfrak{E}$, and $i<\mathfrak{O}-\operatorname{depth}_{\mathfrak{E}}(\beta)$ :

(a) If $i$ is odd, there exists an element $\alpha$ in $\mathfrak{E}$ of $\mathfrak{O}$-depth $i$ such that $\alpha>\beta$.

(b) If $i$ is even and there is no element $\alpha$ in $\mathfrak{E}$ of $\mathfrak{O}$-depth $i$ such that $\alpha>\beta$, then there is element $\alpha$ in $\mathfrak{E}$ of $\mathfrak{O}$-depth $i-1$ such that $p_{h}(\alpha)>\beta$.

Proof Proposition 5.3.4 and Lemma 5.3.2 (4).

Corollary 5.3.6 Let $C$ be a $v$-chain in $\mathfrak{O N}(v)$ with tail $\alpha$ such that $\mathfrak{O}-\operatorname{depth}_{C}(\alpha)$ is odd. Let $A$ be a $v$-chain in $\mathfrak{O N}(v)$ with head $\alpha$, and $D$ the concatenation of $C$ with $A$. Let $C^{\prime}$ denote the $v$-chain $C \backslash\{\alpha\}$. Then

(1) The type of an element of $A$ is the same in both $A$ and D. In particular, $\mathfrak{O m o n}_{A} \subseteq$ $\mathfrak{O m o n}_{D}$ and $\mathfrak{O} q_{A, \beta}=\mathfrak{O} q_{D, \beta}$ for $\beta$ in $A$.

(2) The type of an element of $C^{\prime}$ is the same in both $C^{\prime}$ and $D$. In particular, $\mathfrak{O m o n}_{C^{\prime}} \subseteq \mathfrak{O m o n}_{D}$

(3) $\mathfrak{O m o n}_{D}=\mathfrak{O m o n}_{C^{\prime}} \cup \mathfrak{O m o n}_{A}$ (disjoint union); letting $j_{0}:=\mathfrak{O}$-depth ${ }_{C}(\alpha)$ we have $\left(\mathfrak{O m o n}_{D}\right)^{j_{0}}=\mathfrak{O m o n}_{A}$ and $\left(\mathfrak{O m o n}_{D}\right)_{1} \cup \cdots \cup\left(\mathfrak{O m o n}_{D}\right)_{j_{0}-1}=\mathfrak{O m o n}_{C^{\prime}}$. (For a monomial $\mathfrak{E}$ in $\mathfrak{N}(v)$, the subset of elements of depth at least $i$ is denoted $\mathfrak{E}^{i}$, and the subset of elements of depth exactly $i$ is denoted $\mathfrak{E}_{i}$. )

Proof (1) Generally (meaning without the assumption that $\mathfrak{O}$-depth ${ }_{C}(\alpha)$ is odd), the only element of $A$ that could possibly have a different type in $D$ is the last one in the first connected component of $A$; whether or not it changes type depends exactly upon whether or not the parity of the cardinality of its connected component in $D$ is different from that in $A$. Under our hypothesis, this parity does not change, for, by (3) of Lemma 5.3.2, the type of $\alpha$ in $C$ is $\mathrm{H}$ or $\mathrm{S}$, and so the cardinality of the connected component of $\alpha$ in $C$ is odd.

(2) Generally (meaning without the assumption that $\mathfrak{O}$-depth ${ }_{C}(\alpha)$ is odd), the only element of $C^{\prime}$ that could possibly have a different type in $D$ is the last one of $C^{\prime}$; it changes type if and only if it is connected to $\alpha$ and the cardinality of its connected component in $C^{\prime}$ is odd. Under our hypothesis, this cardinality is even, for the same reason as in (1).

(3) That $\mathfrak{O m o n} \mathfrak{m}_{D}=\mathfrak{O m o n}_{C^{\prime}} \cup \mathfrak{O m o n} \mathfrak{m}_{A}$ (disjoint union) is an immediate consequence of (1) and (2). By Lemma 5.3.2 (1), $\mathfrak{O} q_{A, \alpha}=\mathfrak{O} q_{D, \alpha}$ dominates every ele-

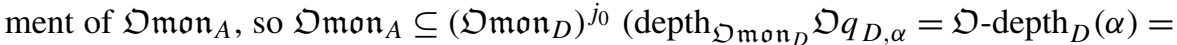
$\mathfrak{O}$-depth $\left.C_{C}(\alpha)=j_{0}\right)$. It is enough to prove the following claim: every element of $\mathfrak{O m o n} C_{C^{\prime}}$ has depth less than $j_{0}$ in $\mathfrak{O m o n}_{D}$. Let $\gamma^{\prime}$ be an element of $\mathfrak{O M m o n}_{C^{\prime}}$. If $\gamma^{\prime}>\mathfrak{O} q_{D, \alpha}$ then the claim is clear. If not, then, by Proposition 5.1.4 (1), $\gamma^{\prime}=p_{h}(\gamma)$. By Lemma 5.3.2 (3), $\mathfrak{O}-\operatorname{depth}_{D}(\gamma)$ is odd. Since the claim is already true for $\mathfrak{O} q_{D, \gamma}=p_{v}(\gamma)$, we have $\mathfrak{O}-\operatorname{depth}_{D}(\gamma)=\operatorname{depth}_{\mathfrak{O m o n}_{D}} p_{v}(\gamma) \leq j_{0}-2$. By (5) of the same lemma, depth $\mathfrak{O m o n}_{D} \gamma^{\prime}=\mathfrak{O}$-depth ${ }_{D}(\gamma)+1$, so depth ${ }_{D} \gamma^{\prime} \leq j_{0}-1$, and the claim is proved. 
Proposition 5.3.7 Let $\mathfrak{E}$ be a monomial in $\mathfrak{O N}(v)$ and $j$ an odd integer. For $\beta$ in $\mathfrak{O E}^{j, j+1}(:=\{\alpha \in \mathfrak{E} \mid \mathfrak{O}$-depth $\mathfrak{E}(\alpha) \geq j\})$, we have

$$
\mathfrak{O} \text {-depth } \mathfrak{O E}^{j, j+1}(\beta)=\mathfrak{O}-\operatorname{depth}_{\mathfrak{E}}(\beta)-j+1
$$

Proof Proceed by induction on $j$. For $j=1$, the assertion reduces to a tautology. Suppose that the assertion has been proved up to $j$. By the induction hypothesis, we have $\mathfrak{O} \mathfrak{E}^{j+2, j+3}=\mathfrak{O}\left(\mathfrak{O} \mathfrak{E}^{j, j+1}\right)^{3,4}$, and we are reduced to proving the assertion for $j=3$.

Let $A$ be a $v$-chain in $\mathfrak{O} \mathfrak{E}^{3,4}$ with tail $\beta$ and $\mathfrak{O}$-depth $\operatorname{dep}_{A}(\beta)=\mathfrak{O}$-depth $\mathfrak{O E}_{\mathfrak{E}, 4}(\beta)$. Let $\alpha$ be the head of $A$. We may assume $\mathfrak{D}-\operatorname{depth}_{\mathfrak{E}}(\alpha)=3$ for, if $\mathfrak{D}-\operatorname{depth}_{\mathfrak{E}}(\alpha)>3$, we can find, by Lemma 5.3.2 (4), $\alpha^{\prime}$ of $\mathfrak{O}$-depth 3 in $\mathfrak{E}$ with $\alpha^{\prime}>\alpha$, and extending $A$ by $\alpha^{\prime}$ will not decrease the $\mathfrak{O}$-depth in $A$ of $\beta$ (Proposition 5.2.1 (1)). Let $E$ be a $v$-chain in $\mathfrak{O} \mathfrak{m o n}_{A}$ with tail $\mathfrak{O} q_{A, \beta}$ and length $\mathfrak{O}$-depth ${ }_{A}(\beta)$. The head of $E$ is then $\mathfrak{O} q_{A, \alpha}$ (see Proposition 5.1.4 (1)).

Choose $C$ in $\mathfrak{E}$ with tail $\alpha$ such that $\mathfrak{O}$-depth $\operatorname{den}_{C}(\alpha)=3$. Let $D$ be the concatenation of $C$ with $A$. By Corollary 5.3.6, $E$ is contained in $\mathfrak{O m o n} \mathfrak{m}_{D}, \mathfrak{O} q_{D, \alpha}=\mathfrak{O} q_{A, \alpha}$, and $\mathfrak{O} q_{D, \beta}=\mathfrak{O} q_{A, \beta}$. By Proposition 5.2.1 (2), the $\mathfrak{O}$-depth of $\alpha$ is the same in $D$ as in $C$. Choose a $v$-chain $F$ in $\mathfrak{O m o n}{ }_{D}$ with tail $\mathfrak{O} q_{D, \alpha}=\mathfrak{O} q_{A, \alpha}$. Concatenating $F$ with $E$ we get a $v$-chain in $\mathfrak{O m o n} \mathfrak{m}_{D}$ with tail $\mathfrak{O} q_{D, \beta}=\mathfrak{O} q_{A, \beta}$ of length $\mathfrak{O}$-depth $\operatorname{OE}_{\mathfrak{E}, 4}(\beta)+$

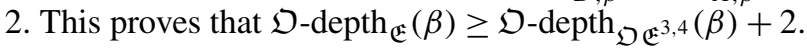

To prove the reverse inequality, we need only turn the above proof on its head. Let $D$ be a $v$-chain in $\mathfrak{E}$ with tail $\beta$ such that $\mathfrak{O}$-depth $\operatorname{Ed}_{\mathfrak{E}}(\beta)=\mathfrak{O}$-depth $\operatorname{dep}_{D}(\beta)$. Let $G$ be a $v$-chain in $\mathfrak{O m o n} \mathfrak{m}_{D}$ with tail $\mathfrak{O} q_{D, \beta}$ and length $\mathfrak{O}$-depth $\mathfrak{E}_{\mathfrak{E}}(\beta)$. There exists an element $\alpha$ in $D$ of $\mathfrak{O}$-depth 3 in $D$ (by Lemma 5.3.2 (4)). Let $C$ be the part of $D$ up to and including $\alpha$, and $A$ the part $\alpha>\ldots>\beta$. By Proposition 5.2.1 (2), $\mathfrak{D}-\operatorname{depth}_{C}(\alpha)=3$ and, as above, Corollary 5.3.6 applies.

By Corollary 5.2.4, $\mathfrak{O} q_{A, \alpha}=\mathfrak{O} q_{D, \alpha}$ occurs in $G$. The part $F$ of $G$ up to and including $\mathfrak{O} q_{A, \alpha}$ is of length at most 3, and the part $E: \mathfrak{O} q_{D, \alpha}>\ldots>\mathfrak{O} q_{D, \beta}$ belongs also to $\mathfrak{D m o n}_{A}$ (Proposition 5.1.4 (2)). Thus the length of $G$ is at most 2 more than the the length of $E$ which is at most $\mathfrak{O}$-depth $\mathfrak{O E}^{3,4}(\beta)$.

Corollary 5.3.8 $\mathfrak{O}\left(\mathfrak{O} \mathfrak{E}^{i, i+1}\right)^{j, j+1}=\mathfrak{O} \mathfrak{E}^{i+j-1, i+j}$ for odd integers $i, j$.

\section{The map $\mathfrak{D} \pi$}

This section describes the map $\mathfrak{D} \pi$. The description is given in $\S 6.1$. It relies on certain claims proved in $\S \S 6.3,6.4$. Those proofs in turn refer to results from $\S 8$ (but there is no circularity): to postpone the definition of $\mathfrak{O} \pi$ until all the results needed for it have been proved would hurt readability. The observations in $\S 6.5$ are required only in $§ 9$.

The symbol $j$ will be reserved for an odd positive integer throughout this section.

\subsection{Description of $\mathfrak{O} \pi$}

The map $\mathfrak{O} \pi$ takes as input a monomial $\mathfrak{E}$ in $\mathfrak{O N}(v)$ and produces as output a pair $\left(\mathfrak{O} w_{\mathfrak{E}}, \mathfrak{O} \Pi_{\mathfrak{E}}\right)$, where $\mathfrak{O} w_{\mathfrak{E}}$ is an element of $\mathfrak{O} I(d)$ such that $\mathfrak{O} w_{\mathfrak{E}} \geq v$ 
and $\mathfrak{O} \Pi_{\mathfrak{E}}$ is a 'smaller' monomial, possibly empty, in $\mathfrak{O N}(v)$. If the input $\mathfrak{E}$ is empty, no output is produced (by definition). So now suppose that $\mathfrak{E}$ is non-empty.

We first partition $\mathfrak{E}$ into subsets according to the $\mathfrak{O}$-depths of its elements. Let $\mathfrak{O C} \mathfrak{E}_{k}^{\mathrm{pr}}$ be the sub-monomial of $\mathfrak{E}$ consisting of those elements of $\mathfrak{E}$ that have $\mathfrak{O}$ depth $k$-the superscript "pr" is short for "preliminary". It follows from Corol-

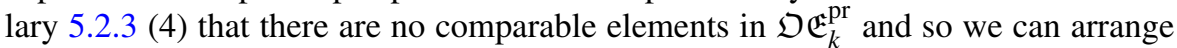
the elements of $\mathfrak{O} \mathfrak{E}_{k}^{\mathrm{pr}}$ in ascending order of both row and column indices. Let $\sigma_{k}$ be the last element of $\mathfrak{O} \mathfrak{E}_{k}^{\mathrm{pr}}$ in this arrangement (assuming $\mathfrak{O} \mathfrak{E}_{k}^{\mathrm{pr}}$ is non-empty).

Let now $j$ be an odd integer. We set $\mathfrak{O C} \mathfrak{E}_{j, j+1}^{\mathrm{pr}}:=\mathfrak{O} \mathfrak{E}_{j}^{\mathrm{pr}} \cup \mathfrak{O} \mathfrak{E}_{j+1}^{\mathrm{pr}}$. We say that $\mathfrak{E}$ is truly orthogonal at $j$ if $\mathfrak{O} \mathfrak{E}_{j, j+1}^{\mathrm{pr}}$ is non-empty (and therefore $\sigma_{j}$ exists-by Lemma 5.3.2 (4a)) and $p_{h}\left(\sigma_{j}\right)$ belongs to $\mathfrak{N}(v)$ (that is, if $r>r^{*}$ where $\sigma_{j}=(r, c)$ ).

Let $\mathfrak{O} \mathfrak{E}_{j, j+1}$ denote the monomial in $\mathfrak{N}(v)$ defined by $\mathfrak{O} \mathfrak{E}_{j, j+1}:=$

$$
\begin{cases}\left(\mathfrak{O E} \mathfrak{E}_{j, j+1}^{\mathrm{pr}} \backslash\left\{\sigma_{j}\right\}\right) \cup\left(\mathfrak{O E} \mathfrak{E}_{j, j+1}^{\mathrm{pr}} \backslash\left\{\sigma_{j}\right\}\right)^{\#} \cup\left\{p_{v}\left(\sigma_{j}\right), p_{h}\left(\sigma_{j}\right)\right\} & \begin{array}{l}
\text { if } \mathfrak{E} \text { is truly } \\
\text { orthogonal at } j
\end{array} \\
\mathfrak{O} \mathfrak{E}_{j, j+1}^{\mathrm{pr}} \cup\left(\mathfrak{\mathcal { O }} \mathfrak{E}_{j, j+1}^{\mathrm{pr}}\right)^{\#} & \text { otherwise }\end{cases}
$$

Here $\mathfrak{O} \mathfrak{E}_{j, j+1}^{\mathrm{pr}} \backslash\left\{\sigma_{j}\right\}$ and other terms on the right are multisets: the multiplicity of $\sigma_{j}$ in $\mathfrak{O} \mathfrak{E}_{j, j+1}^{\mathrm{pr}} \backslash\left\{\sigma_{j}\right\}$ is one less than that in $\mathfrak{O E}_{j, j+1}^{\mathrm{pr}}$. As proved in Corollary 6.3.4 (1) below, $\mathfrak{O} \mathfrak{E}_{j, j+1}$ has depth at most 2 . Let $\mathfrak{O} \mathfrak{E}_{j}$ (respectively $\mathfrak{O} \mathfrak{E}_{j+1}$ ) be the subset (as a multiset) of elements of depth 1 (respectively 2) of $\mathfrak{O} \mathfrak{E}_{j, j+1}$.

Now, for every integer $k$, we apply the map of $\pi$ of $[7, \S 4]$ to $\mathfrak{O} \mathfrak{E}_{k}$ to obtain a pair $\left(\mathfrak{O} w_{\mathfrak{E}}(k), \mathfrak{O} \mathfrak{E}_{k}^{\prime}\right)$, where $\mathfrak{O} w_{\mathfrak{E}}(k)$ is an element of $I(d, 2 d)$ and $\mathfrak{O} \mathfrak{E}_{k}^{\prime}$ is a monomial in $\mathfrak{N}(v)$. Let $\mathfrak{S}_{\mathfrak{O} w_{\mathfrak{E}}(k)}$ be the distinguished monomial in $\mathfrak{N}(v)$ associated to $\mathfrak{O} w_{\mathfrak{E}}(k)$-see $\$ 2.6 .2$.

\section{Proposition 6.1.1}

(1) $\mathfrak{S}_{\mathfrak{O} w_{\mathfrak{E}}(k),} \mathfrak{O} \mathfrak{E}_{k}^{\prime}$ are symmetric; therefore $\cup_{k} \mathfrak{S}_{\mathfrak{O} w_{\mathfrak{E}}(k)}, \cup_{k} \mathfrak{O} \mathfrak{E}_{k}^{\prime}$ are so too.

(2) $\cup_{k} \mathfrak{S}_{\mathfrak{O} w_{\mathfrak{E}}(k)}$ is a distinguished subset of $\mathfrak{N}(v)$ (in particular, the $\mathfrak{S}_{\mathfrak{O} w_{\mathfrak{E}}(k)}$ are disjoint).

(3) For $j$ an odd integer, either both $\mathfrak{S}_{\mathfrak{O} w_{\mathfrak{E}}(j)}$ and $\mathfrak{S}_{\mathfrak{O} w_{\mathfrak{E}}(j+1)}$ meet the diagonal, or neither of them does, precisely as whether or not $\mathfrak{E}$ is truly orthogonal at $j$. And therefore $\cup_{k} \mathfrak{S}_{\mathfrak{O} w_{\mathfrak{E}}(k)}$ has evenly many diagonal elements.

(4) No $\mathfrak{O} \mathfrak{E}_{k}^{\prime}$ intersects the diagonal. And therefore neither does $\cup_{k} \mathfrak{D} \mathfrak{E}_{k}^{\prime}$.

The proposition will be proved below in $\$ 6.4$.

We are finally ready to define the image $\left(\mathfrak{O} w_{\mathfrak{E}}, \mathfrak{O} \Pi_{\mathfrak{E}}\right)$ of $\mathfrak{E}$ under $\mathfrak{O} \pi$. We let $\mathfrak{O} w_{\mathfrak{E}}$ be the element of $I(d, 2 d)$ associated to the distinguished subset $\cup_{k} \mathfrak{S}_{\mathfrak{O} w_{\mathfrak{E}}(k)}$ of $\mathfrak{N}(v)$; since $\cup_{k} \mathfrak{S}_{\mathfrak{O} w_{\mathfrak{E}}(k)}$ is symmetric and has evenly many diagonal elements, it follows (Proposition 2.6.1) that $\mathfrak{O} w_{\mathfrak{E}}$ is in $\mathfrak{O} I(d)$. And we take $\mathfrak{O} \Pi_{\mathfrak{E}}:=\cup_{k} \mathfrak{O} \mathfrak{E}_{k}^{\prime} \cap \mathfrak{O N}(v)$.

Remark 6.1.2 Setting

$$
\pi\left(\mathfrak{O E} \mathfrak{E}_{j, j+1}\right):=\left(y_{j, j+1}, \mathfrak{O \mathfrak { E } _ { j , j + 1 } ^ { \prime }}\right), \quad \mathfrak{O} \prod_{\mathfrak{E}}:=\cup_{j \text { odd }} \mathfrak{O \mathfrak { E } _ { j , j + 1 } ^ { \prime }} \cap \mathfrak{O N}(v),
$$


and defining $\mathfrak{O} w_{\mathfrak{E}}$ to be the element of $I(d, 2 d)$ associated to $\cup_{j \text { odd }} \mathfrak{S}_{y_{j, j+1}}$ would give an equivalent definition of $\mathfrak{O} \pi$.

\subsection{Illustration by an example}

We illustrate the map $\mathfrak{O} \pi$ by means of an example. Let $d=15$, and $v=$ $(1,2,3,4,9,10,14,16,18,19,20,23,24,25,26)$. A monomial $\mathfrak{E}$ in $\mathfrak{O N}(v)$ is shown in Figure 5. Solid black dots indicate the elements that occur in $\mathfrak{E}$ with nonzero multiplicity. Integers written near the solid dots indicate multiplicities.

The $\mathfrak{O}$-depth of $\mathfrak{E}$ is 5 . The element $(21,9)$ has $\mathfrak{O}$-depth 3 although it has depth 2 in $\mathfrak{E}$. Figure 6 shows the monomials $\mathfrak{O} \mathfrak{E}_{1,2}^{\mathrm{pr}}, \mathfrak{O} \mathfrak{E}_{3,4}^{\mathrm{pr}}$, and $\mathfrak{O} \mathfrak{E}_{5,6}^{\mathrm{pr}}$. Solid dots, open dots, and crosses indicate elements of these monomials respectively. The monomial $\mathfrak{E}$ is truly orthogonal at 1 and 3 but not at 5: $\sigma_{1}=(28,2), \sigma_{3}=(21,9)$, and $\sigma_{5}=(15,14)$.

Figure 7 shows the monomials $\mathfrak{O E}_{1,2}, \mathfrak{O} \mathfrak{E}_{3,4}$, and $\mathfrak{O} \mathfrak{E}_{5,6}$ of $\mathfrak{N}(v)$ and also their

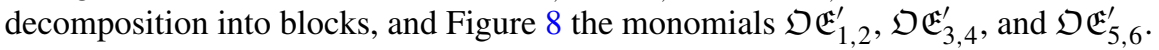

We have

$$
\cup_{k} \mathfrak{S}_{\mathfrak{O} w_{\mathfrak{E}}(k)}=\{(15,14),(17,16),(21,10),(7,4),(27,24),(28,3),(30,1),(29,2)\}
$$

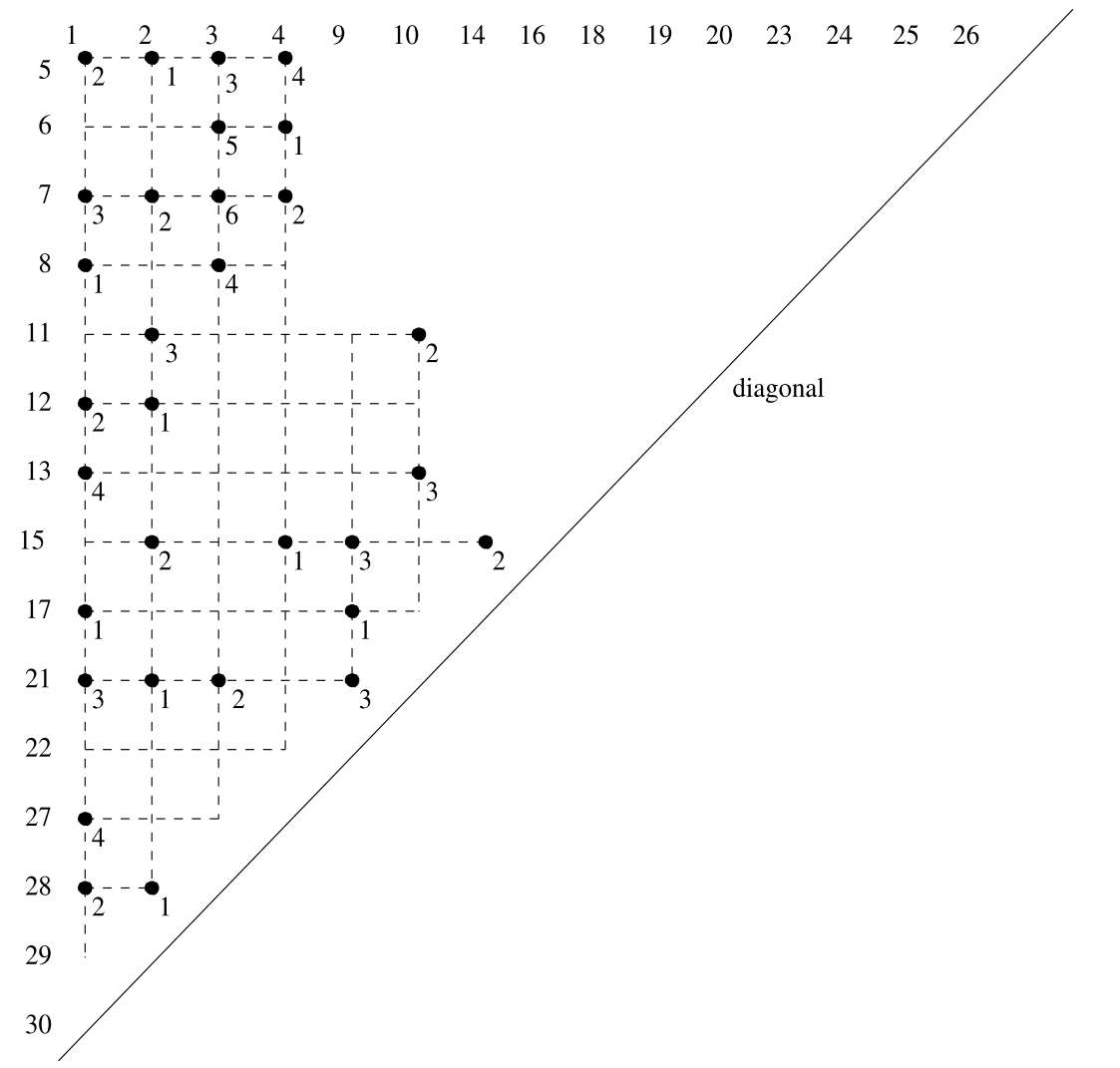

Fig. 5 The monomial $\mathfrak{E}$ 


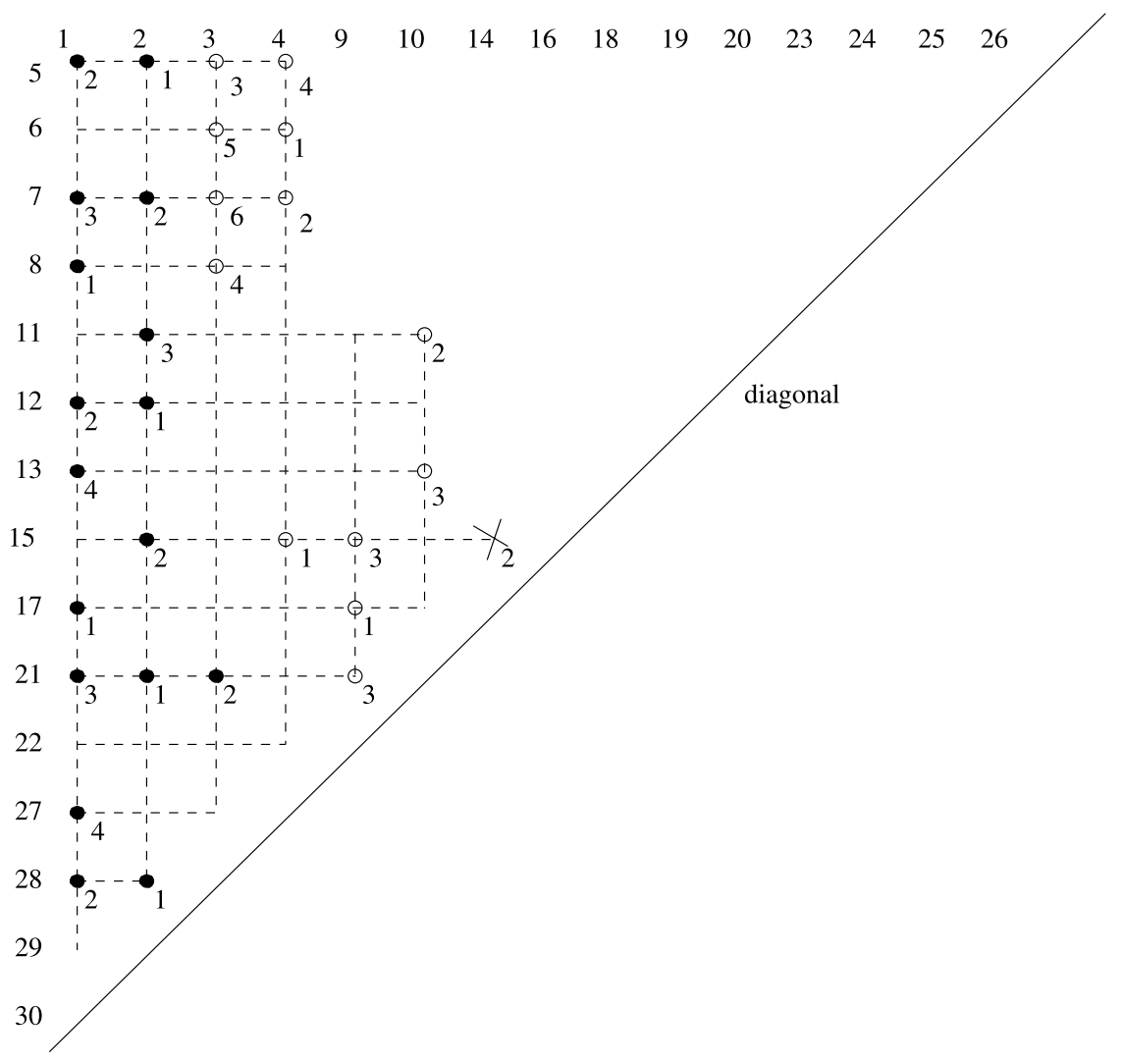

Fig. $6 \mathfrak{O E} \mathfrak{E}_{1,2}^{\mathrm{pr}}, \mathfrak{O} \mathfrak{E}_{3,4}^{\mathrm{pr}}$, and $\mathfrak{O} \mathfrak{E}_{5,6}^{\mathrm{pr}}$

hence $\mathfrak{O} w_{\mathfrak{E}}=(7,9,15,17,18,19,20,21,23,25,26,27,28,29,30)$. The monomial $\mathfrak{O} \Pi_{\mathfrak{E}}$ is the intersection with $\mathfrak{O N}(v)$ of the union of $\mathfrak{O \mathfrak { E } _ { 1 , 2 } ^ { \prime }}, \mathfrak{O \mathfrak { E } _ { 3 , 4 } ^ { \prime }}$, and $\mathfrak{O} \mathfrak{E}_{5,6}^{\prime}$-in other words it is just the monomial lying above $\mathfrak{d}$ in Figure 8.

\subsection{A proposition about $\mathfrak{O} \mathfrak{E}_{j, j+1}$}

The aim of this subsection is to show that $\mathfrak{O E}_{j, j+1}$ has depth no more than 2 - see item (b) of Proposition 6.3.3. This basic fact was mentioned above in the description of $\mathfrak{O} \pi$ and is necessary (psychologically although not logically) to make sense of the definitions of $\mathfrak{O E} \mathfrak{E}_{j}$ and $\mathfrak{O} \mathfrak{E}_{j+1}$. We prepare the way for Proposition 6.3 .3 by way of two preliminary propositions. The first of these is about elements of $\mathfrak{O}$-depth $j$ and $j+1$ in $\mathfrak{E}$, the second about the relation of these elements with $\sigma_{j}$.

\section{Proposition 6.3.1}

(1) $\mathfrak{O E}_{k}^{\mathrm{pr}}$ has no comparable elements. 


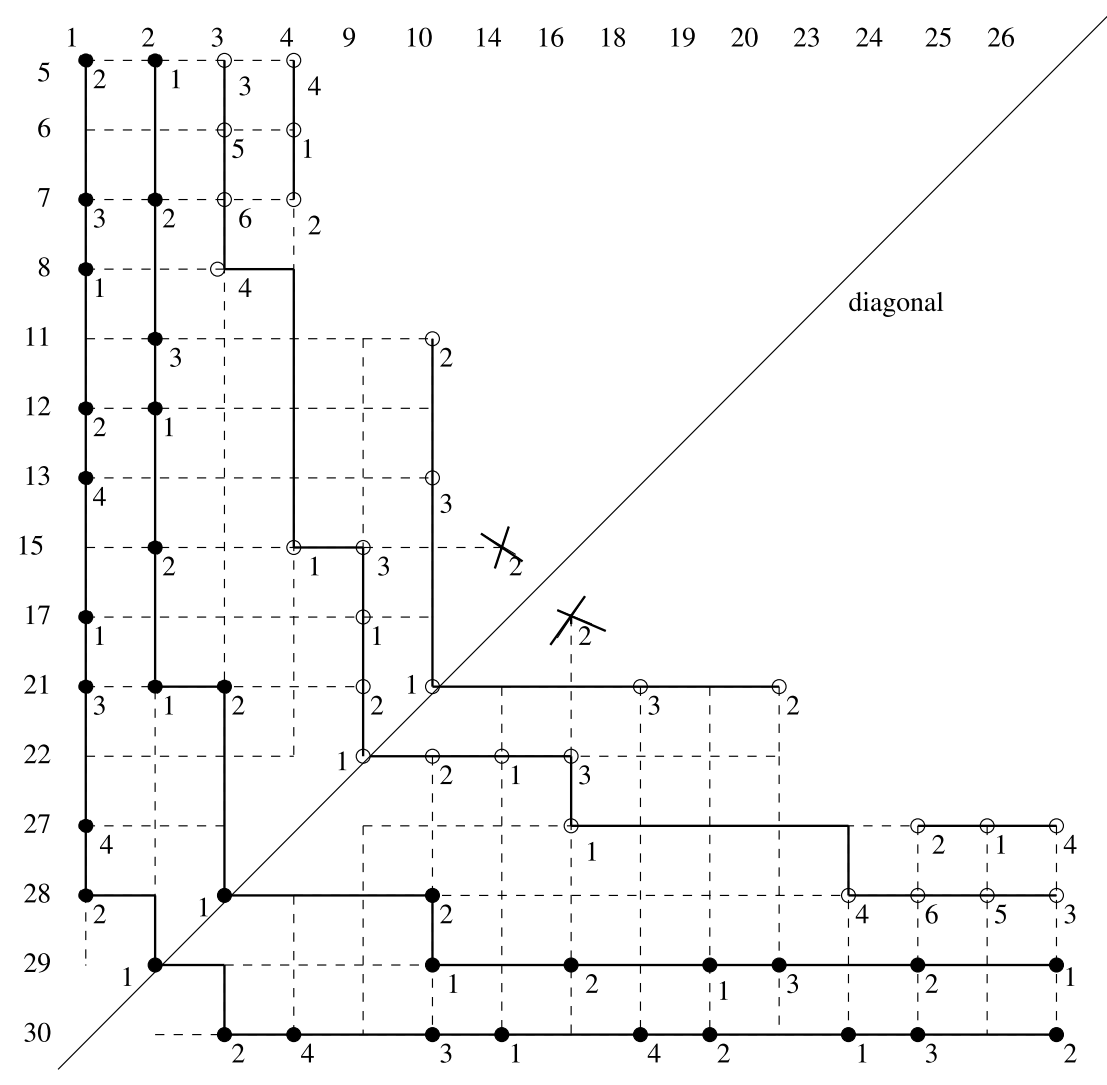

Fig. $7 \mathfrak{O E}_{1,2}, \mathfrak{O E} 3,4$, and $\mathfrak{O E} \mathfrak{E}_{5,6}$

(2) For $j$ an odd integer and $\beta$ an element of $\mathfrak{O E}_{j+1}^{\mathrm{pr}}$, there exists $\alpha$ in $\mathfrak{O E}_{j}^{\mathrm{pr}}$ such that $\alpha>\beta$. In particular, the row index of $\sigma_{j+1}\left(\right.$ if $\sigma_{j+1}$ exists) is less than the row index of $\sigma_{j}$.

Proof For (1) use Corollary 5.2.3 (4); for (2), Prop. 5.3.4, Lemma 5.3.2 (4).

Proposition 6.3.2 Let $\mathfrak{E}$ be truly orthogonal at $j$, with $j$ odd. Then

(1) $p_{v}\left(\sigma_{j}\right)>p_{h}\left(\sigma_{j}\right)$; if $\alpha>p_{v}\left(\sigma_{j}\right)$, then $\alpha>\sigma_{j}$; if $\alpha>\sigma_{j}$, then $\alpha>p_{h}\left(\sigma_{j}\right)$.

(2) No element of $\mathfrak{O} \mathfrak{E}_{j}^{\mathrm{pr}}$ is comparable to $p_{v}\left(\sigma_{j}\right)$ or $p_{h}\left(\sigma_{j}\right)$.

(3) No element of $\mathfrak{O} \mathfrak{E}_{j+1}^{\mathrm{pr}}$ is comparable to $p_{h}\left(\sigma_{j}\right)$.

(4) The following is not possible: $\alpha \in \mathfrak{O} \mathfrak{E}_{j}^{\mathrm{pr}}, \beta \in \mathfrak{O} \mathfrak{E}_{j+1}^{\mathrm{pr}}$, and $p_{h}(\alpha)>\beta$.

Proof (1) is trivial. (2) follows immediately from the definition of $\sigma_{j}$. We now prove (3). First suppose $\beta>p_{h}\left(\sigma_{j}\right)$ for some $\beta$ in $\mathfrak{O} \mathfrak{E}_{j+1}^{\mathrm{pr}}$. By (2) of Proposition 6.3.1, there exists $\alpha$ in $\mathfrak{O C} \mathfrak{E}_{j}^{\mathrm{pr}}$ such that $\alpha>\beta$. But then the row index of $\alpha$ exceeds that of $\sigma_{j}$, a contradiction to the choice of $\sigma_{j}$. 


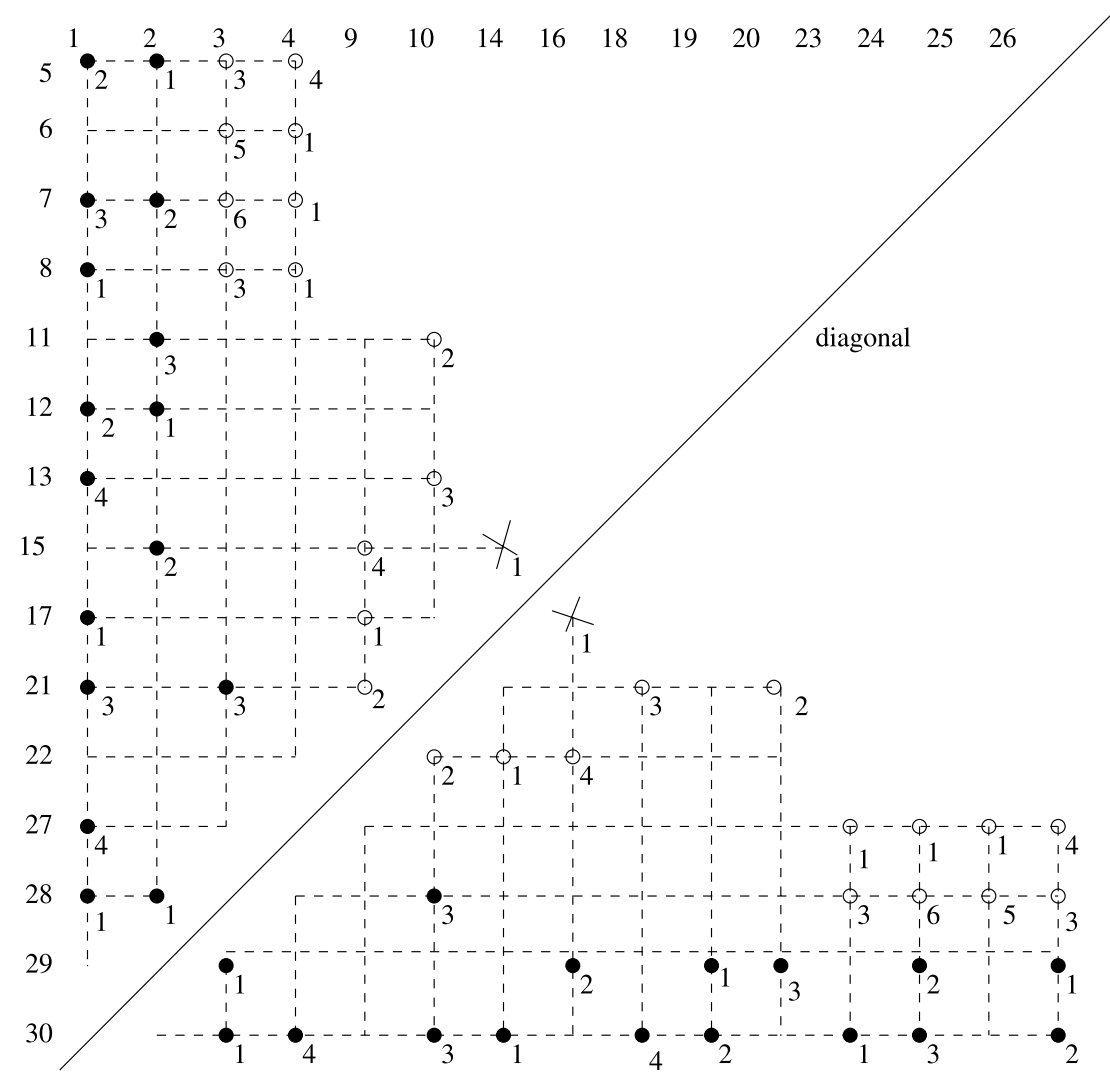

Fig. $8 \mathfrak{O E} \mathfrak{E}_{1,2}^{\prime}, \mathfrak{O E} \mathfrak{E}_{3,4}^{\prime}$, and $\mathfrak{O E} \mathfrak{E}_{5,6}^{\prime}$

We claim it is not possible for $\beta \in \mathfrak{O} \mathfrak{E}_{j+1}^{\mathrm{pr}}$ to satisfy $p_{h}\left(\sigma_{j}\right)>\beta$. This being a special case of (4), we need only prove that statement. So suppose $\alpha$ belongs to $\mathfrak{O} \mathfrak{E}_{j}^{\mathrm{pr}}$ and $p_{h}(\alpha)>\beta$. Let $C$ be a $v$-chain in $\mathfrak{E}$ with tail $\alpha$ and $\mathfrak{O}$-depth $\operatorname{dep}_{C}(\alpha)=j$ (Proposition 5.2.3 (1)). Concatenate $C$ with $\alpha>\beta$ and call the resulting $v$-chain $D$. Then, by Lemma 5.3.2 (3), $\alpha$ is of type $\mathrm{H}$ in $D$, so that, by Lemma 5.3.2 (1), we have $\mathfrak{O}-\operatorname{depth}_{D}(\beta)=\mathfrak{O}-\operatorname{depth}_{D}(\alpha)+2$. But, by Proposition 5.2.1 (2), $\mathfrak{O}-\operatorname{depth}_{D}(\alpha)=$ $\mathfrak{O}$-depth ${ }_{C}(\alpha)=j$, so that $\mathfrak{O}-\operatorname{depth}_{\mathfrak{E}}(\beta) \geq j+2$, a contradiction.

Let $\mathfrak{O} \mathfrak{E}_{j, j+1}(\mathrm{ext})$ denote the set—not multiset—defined by:

$$
\mathfrak{O E} \mathfrak{E}_{j, j+1}(\mathrm{ext}):= \begin{cases}\mathfrak{\mathcal { E }} \mathfrak{E}_{j, j+1} \cup\left\{\sigma_{j}, \sigma_{j}^{\#}\right\} & \text { if } \mathfrak{E}_{\text {is truly orthogonal at } j} \\ \mathfrak{\mathcal { O E }} \mathfrak{E}_{j, j+1} & \text { otherwise }\end{cases}
$$

Here $\mathfrak{O} \mathfrak{E}_{j, j+1}$ on the right stands for the underlying set of the multiset $\mathfrak{O} \mathfrak{E}_{j, j+1}$ defined above. The set $\mathfrak{O} \mathfrak{E}_{j, j+1}(\mathrm{ext})$ is the disjoint union of the sets $\mathfrak{O} \mathfrak{E}_{j}(\mathrm{ext})$ and $\mathfrak{O C} \mathfrak{E}_{j+1}(\mathrm{ext})$ defined as follows (here again the terms on the right hand side denote 
the underlying sets of the corresponding multisets):

$$
\begin{aligned}
& \mathfrak{O E} \mathfrak{E}_{j}(\operatorname{ext}):= \begin{cases}\mathfrak{O} \mathfrak{E}_{j}^{\mathrm{pr}} \cup\left(\mathfrak{O E} \mathfrak{E}_{j}^{\mathrm{pr}}\right)^{\#} \cup\left\{p_{v}(\sigma)\right\} & \text { if } \mathfrak{E} \text { is truly orthogonal at } j \\
\mathfrak{O E} \mathfrak{E}_{j}^{\mathrm{pr}} \cup\left(\mathfrak{O E} \mathfrak{E}_{j}^{\mathrm{pr}}\right)^{\#} & \text { otherwise }\end{cases} \\
& \mathfrak{O E} \mathfrak{E}_{j+1}(\mathrm{ext}):= \begin{cases}\mathfrak{O} \mathfrak{E}_{j+1}^{\mathrm{pr}} \cup\left(\mathfrak{O C E} \mathfrak{E}_{j+1}^{\mathrm{pr}}\right)^{\#} \cup\left\{p_{h}(\sigma)\right\} & \text { if } \mathfrak{E} \text { is truly orthogonal at } j \\
\mathfrak{O} \mathfrak{E}_{j+1}^{\mathrm{pr}} \cup\left(\mathfrak{O C E} \mathfrak{E}_{j+1}^{\mathrm{pr}}\right)^{\#} & \text { otherwise }\end{cases}
\end{aligned}
$$

\section{Proposition 6.3.3}

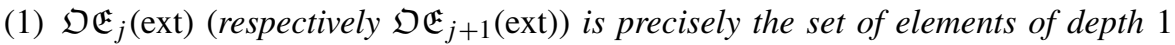
(respectively 2) in $\mathfrak{O} \mathfrak{E}_{j, j+1}(\mathrm{ext})$. In particular,

(a) Neither $\mathfrak{O} \mathfrak{E}_{j}(\mathrm{ext})$ nor $\mathfrak{O} \mathfrak{E}_{j+1}(\mathrm{ext})$ contains comparable elements.

(b) The length of a $v$-chain in $\mathfrak{O E}_{j, j+1}(\mathrm{ext})$ is at most 2.

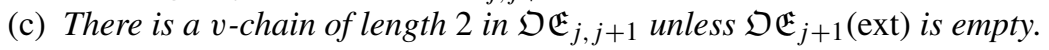

(2) Let $k$ be a positive integer, not necessarily odd. If there is in $\mathfrak{E}$ an element of $\mathfrak{O}$-depth at least $k$, then $\mathfrak{O E}_{k}(\mathrm{ext})$ is non-empty. The converse also holds except possibly if $k$ is even and $\mathfrak{E}$ is truly orthogonal at $k-1$. In particular, if $\mathfrak{O} \mathfrak{E}_{k}(\mathrm{ext})$ is non-empty, then there is an element of $\mathfrak{O}$-depth at least $k-1$.

Proof (1): It is enough to show that every element of $\mathfrak{O E}_{j}(\operatorname{ext})(\mathrm{up})$ (respectively $\mathfrak{D E} \mathfrak{E}_{j+1}\left(\right.$ ext)(up)) is of depth 1 (respectively 2) in $\mathfrak{O} \mathfrak{E}_{j, j+1}($ ext)(up), for

- $\alpha>\beta$ implies $\alpha$ (up) $>\beta$ (up) for elements $\alpha, \beta$ of $\mathfrak{N}(v)$.

- $\mathfrak{O} \mathfrak{E}_{j, j+1}($ ext $)=\mathfrak{O} \mathfrak{E}_{j}($ ext $) \cup \mathfrak{O} \mathfrak{E}_{j+1}($ ext $)$.

- $\mathfrak{O} \mathfrak{E}_{j, j+1}(\mathrm{ext}), \mathfrak{O} \mathfrak{E}_{j}(\mathrm{ext})$, and $\mathfrak{O} \mathfrak{E}_{j+1}(\mathrm{ext})$ are symmetric.

In turn, it is enough to show the following:

(i) Every element of $\mathfrak{O \mathfrak { E } _ { j }}$ (ext)(up) has depth 1.

(ii) $\mathfrak{O} \mathfrak{E}_{j+1}($ ext)(up) has no comparable elements.

(iii) Every element of $\mathfrak{O} \mathfrak{E}_{j+1}($ ext)(up) has depth at least 2.

Item (i) follows from Proposition 6.3.1 and Proposition 6.3.2 (2); item (ii) from Proposition 6.3.1 (1) and Proposition 6.3.2 (3); item (iii) from Proposition 6.3.1 (2) and Proposition 6.3.2 (1).

(2): The first assertion follows from Lemma 5.3.2 (4): if $k$ is odd there is an element of $\mathfrak{O}$-depth $k$ in $\mathfrak{E}$; if $k$ is even and there is no element of $\mathfrak{O}$-depth $k$ in $\mathfrak{E}$, then there is in $\mathfrak{E}$ an element of $\mathfrak{O}$-depth $k-1$ and type $\mathrm{H}$, so $\mathfrak{E}$ is truly orthogonal at $k-1$. The second assertion is clear from the definition of $\mathfrak{O} \mathfrak{E}_{k}(\mathrm{ext})$.

\section{Corollary 6.3.4}

(1) No element of $\mathfrak{O} \mathfrak{E}_{j, j+1}$ has depth more than 2 .

(2) $\mathfrak{O E}_{j+1}(\mathrm{ext})=\mathfrak{O} \mathfrak{E}_{j+1}$ and $\mathfrak{O E} \mathfrak{E}_{j}(\mathrm{ext}) \cap \mathfrak{O E} \mathfrak{E}_{j, j+1}=\mathfrak{O} \mathfrak{E}_{j}$ (as sets). In particular, $\mathfrak{O E} \mathfrak{E}_{j+1}=\mathfrak{O E}_{j, j+1} \cap \mathfrak{O E E} \mathfrak{E}_{j+1}(\mathrm{ext})$ and $\mathfrak{O E} \mathfrak{E}_{j}=\mathfrak{O E} \mathfrak{E}_{j, j+1} \cap \mathfrak{O E} \mathfrak{E}_{j}(\mathrm{ext})$ as multisets defined by the intersection of a multiset with a subset. 


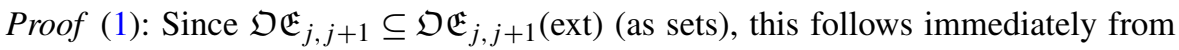
item (b) of the proposition above.

(2): Since the union of $\mathfrak{O} \mathfrak{E}_{j+1}\left(\right.$ ext) (which always is contained in $\mathfrak{O} \mathfrak{E}_{j, j+1}$ ) and $\mathfrak{O E} \mathfrak{E}_{j}(\mathrm{ext}) \cap \mathfrak{O E}_{j, j+1}$ is all of $\mathfrak{O E} \mathfrak{E}_{j, j+1}$, and since $\mathfrak{O E _ { j }}, \mathfrak{O E} \mathfrak{E}_{j+1}$ are disjoint, it it enough to show that $\mathfrak{O E} \mathfrak{E}_{j+1}($ ext $) \subseteq \mathfrak{O E} \mathfrak{E}_{j+1}$ and $\mathfrak{O E} \mathfrak{E}_{j}($ ext $) \cap \mathfrak{O E} \mathfrak{E}_{j, j+1} \subseteq \mathfrak{O E}_{j}$

Now, since elements of $\mathfrak{O} \mathfrak{E}_{j}\left(\right.$ ext) have depth 1 even in $\mathfrak{O} \mathfrak{E}_{j, j+1}$ (ext) (by item (1) of the proposition above), it is immediate that $\mathfrak{O E}_{j}(\mathrm{ext}) \cap \mathfrak{O} \mathfrak{E}_{j, j+1} \subseteq \mathfrak{O} \mathfrak{E}_{j}$. And it follows from the proof of item (iii) in the proof of item (1) of the proposition above that an element of $\mathfrak{O E} \mathfrak{E}_{j+1}\left(\right.$ ext) has depth 2 even in $\mathfrak{O} \mathfrak{E}_{j, j+1}$ (not just in $\mathfrak{O E} \mathfrak{E}_{j, j+1}($ ext $\left.)\right)$, so that $\mathfrak{O E} \mathfrak{E}_{j+1}($ ext $) \subseteq \mathfrak{O} \mathfrak{E}_{j+1}$.

\subsection{Proof of Proposition 6.1.1}

(1) The monomials $\mathfrak{O} \mathfrak{E}_{j, j+1}$ are clearly symmetric. Observe that $\alpha$ in $\mathfrak{O} \mathfrak{E}_{j, j+1}$ has the same depth as $\alpha^{\#}$, for $\alpha_{1}>\alpha_{2}$ implies $\alpha$ (up) $>\alpha_{2}$ (up) and $\alpha$ (down) $>\alpha_{2}$ (down) for $\alpha_{1}, \alpha_{2}$ in $\mathfrak{N}(v)$. Thus the monomials $\mathfrak{O E}_{k}$ are symmetric. Since the map $\pi$ of [7] respects \#-see Proposition 5.7 of [4]-it follows that $\mathfrak{S}_{\mathfrak{O} w_{\mathfrak{E}}(k)}$ and $\mathfrak{O} \mathfrak{E}_{k}^{\prime}$ are symmetric. Therefore so are $\cup_{k} \mathfrak{S}_{\mathfrak{O} w_{\mathfrak{E}}(k)}$ and $\cup_{k} \mathfrak{O} \mathfrak{E}_{k}^{\prime}$.

(2) This follows from Corollary 8.3.6.

(3) If $\mathfrak{E}$ is truly orthogonal at $j$, then $p_{v}\left(\sigma_{j}\right)$ and $p_{h}\left(\sigma_{j}\right)$ are diagonal elements respectively in $\mathfrak{O E} \mathfrak{E}_{j}$ and $\mathfrak{O} \mathfrak{E}_{j+1}$-see Corollary 6.3.4 (2). Thus both $\mathfrak{O} \mathfrak{E}_{j}$ and $\mathfrak{O E} \mathfrak{E}_{j+1}$ have diagonal blocks in the sense of Proposition 5.10 (A) of [4]. It follows from the result just quoted that both $\mathfrak{S}_{\mathfrak{O} w_{\mathfrak{E}}(j)}$ and $\mathfrak{S}_{\mathfrak{O} w_{\mathfrak{E}}(j+1)}$ meet the diagonal. It is of course clear that each $\mathfrak{S}_{\mathfrak{O} w_{\mathfrak{E}}(k)}$ meets the diagonal at most once since diagonal elements are clearly comparable but elements of $\mathfrak{S}_{\mathfrak{O} w_{\mathfrak{E}}(k)}$ are not by Lemma 4.9 of [7].

Suppose that $\mathfrak{E}$ is not truly orthogonal at $j$. Then $\sigma_{j}$ and $\sigma_{j}^{\#}$ belong to different blocks - this is equivalent to the definition of $\mathfrak{E}$ being not truly orthogonal at $j$. By Proposition 6.3.1 (2), it follows that $\sigma_{j+1}$ and $\sigma_{j+1}^{\#}$ also belong to different blocks. So neither $\mathfrak{O} \mathfrak{E}_{j}$ nor $\mathfrak{O} \mathfrak{E}_{j+1}$ has a diagonal block.

(4) If $\mathfrak{E}$ is not truly orthogonal at $j$, then neither $\mathfrak{O} \mathfrak{E}_{j}$ nor $\mathfrak{O} \mathfrak{E}_{j+1}$ has a diagonal block (as has just been said above), and it follows from Proposition 5.10 (A) of [4] that neither $\mathfrak{O} \mathfrak{E}_{j}^{\prime}$ nor $\mathfrak{O} \mathfrak{E}_{j+1}^{\prime}$ meets the diagonal.

So suppose that $\mathfrak{E}$ is truly orthogonal at $j$. Then both $\mathfrak{D E} \mathfrak{E}_{j}$ and $\mathfrak{D E} \mathfrak{E}_{j+1}$ have a diagonal entry each of multiplicity 1 , namely $p_{v}\left(\sigma_{j}\right)$ and $p_{h}\left(\sigma_{j}\right)$ respectively. It is clear from the definition of $\sigma_{j}$ that no element of $\mathfrak{O} \mathfrak{E}_{j}$ (up) shares its row index with $p_{v}\left(\sigma_{j}\right)$. And it follows from Proposition 6.3.1 (2) that no element of $\mathfrak{O E} \mathfrak{E}_{j+1}$ (up) shares its row index with $p_{h}\left(\sigma_{j}\right)$. It now follows from the proof of Proposition 5.10 (B) of [4] - see the last line of that proof-that neither $\mathfrak{O} \mathfrak{E}_{j}^{\prime}$ nor $\mathfrak{O} \mathfrak{E}_{j+1}^{\prime}$ meets the diagonal.

\subsection{More observations}

It follows from Corollary 6.3 .4 (1) that the length of any $v$-chain in $\mathfrak{O E}_{j, j+1}$ is at most 2. Applying Lemma 8.1.1 to $\mathfrak{O} \mathfrak{E}_{j, j+1}$, we get:

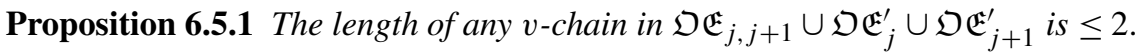




\section{Proposition 6.5.2}

(1) For $\alpha^{\prime}=(r, c)$ in $\mathfrak{O} \mathfrak{E}_{k}^{\prime}(\mathrm{up})$, there exists $\alpha=(r, C)$ in $\mathfrak{O} \mathfrak{E}_{k}^{\mathrm{pr}}$ with $C \leq c$.

(2) For $\alpha^{\prime}=(r, c)$ in $\mathfrak{D} \mathfrak{E}_{j+1}^{\prime}$ (up), there exists $\alpha=(R, c)$ in $\mathfrak{O} \mathfrak{E}_{j+1}$ (up) with $r \leq R$.

(3) For $\alpha^{\prime}$ in $\mathfrak{O \mathfrak { E } _ { j + 1 } ^ { \prime }}{ }_{j}$ (up), there exists $\alpha$ in $\mathfrak{O} \mathfrak{E}_{j}^{\mathrm{pr}}$ with $\alpha>\alpha^{\prime}$.

Proof (1) That there exists $\alpha$ in $\mathfrak{O} \mathfrak{E}_{k}$ (up) with $C \leq c$ follows from the definition of $\mathfrak{O} \mathfrak{E}_{k}^{\prime}$ (up). Clearly such an $\alpha$ cannot be on the diagonal, so $\alpha$ belongs to $\mathfrak{O} \mathfrak{E}_{k}^{\mathrm{pr}}$.

(2) As in the proof of (1), it follows from the definition of $\mathfrak{O} \mathfrak{E}_{j+1}^{\prime}$ that there exists $\alpha=(R, c)$ in $\mathfrak{O E _ { j + 1 }}$ with $r \leq R$. If $\alpha$ lies strictly below the diagonal, then $c>R^{*}$, so that $\alpha^{*}=\left(c^{*}, R^{*}\right)>\alpha^{\prime}=(r, c)$, a contradiction to Lemma 8.1.1 ( $\alpha^{*}$ belongs to $\mathfrak{O E} \mathfrak{E}_{j+1}$ by the symmetry of $\mathfrak{O E} \mathfrak{E}_{j+1}$ ). Thus $\alpha$ belongs to $\mathfrak{O E} \mathfrak{E}_{j+1}$ (up).

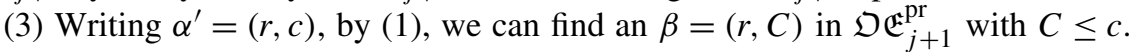
By Proposition 6.3.1 (2), there exists $\alpha$ in $\mathfrak{O} \mathfrak{E}_{j, j+1}^{\mathrm{pr}}$ such that $\alpha>\beta$.

Corollary 6.5.3 If in $\mathfrak{O} \mathfrak{E}_{j}^{\prime}$ (up) $\cup \mathfrak{O} \mathfrak{E}_{j+1}^{\prime}$ (up) there exists an element with horizontal projection in $\mathfrak{N}(v)$, then $\mathfrak{E}$ is truly orthogonal at $j$.

Proposition 6.5.4 The $\mathfrak{O}$-depth of an element in $\mathfrak{O E}_{j}^{\mathrm{pr}} \cup \mathfrak{O} \mathfrak{E}_{j+1}^{\mathrm{pr}}$ is at most 2. More strongly, the $\mathfrak{O}$-depth of an element in $\mathfrak{O} \mathfrak{E}_{j, j+1}^{\mathrm{pr}} \cup \mathfrak{O} \mathfrak{E}_{j}^{\prime}$ (up) $\cup \mathfrak{O} \mathfrak{E}_{j+1}^{\prime}$ (up) is at most 2.

Proof It is enough to show that no element in $\mathfrak{O \mathfrak { E } _ { j } ^ { \prime }}{ }_{j}$ up) $\cup \mathfrak{O} \mathfrak{E}_{j+1}^{\prime}$ (up) has $\mathfrak{O}$-depth more than 2, for we may assume by increasing multiplicities that $\mathfrak{O \mathfrak { E } _ { j } ^ { \mathrm { pr } }} \subseteq \mathfrak{\mathcal { O E }}{ }_{j}^{\prime}$ (up) and $\mathfrak{O C} \mathfrak{E}_{j+1}^{\mathrm{pr}} \subseteq \mathfrak{O} \mathfrak{E}_{j+1}^{\prime}$ (up) (as sets). It follows from Proposition 6.5.1 that a $v$-chain in $\mathfrak{O E} \mathfrak{E}_{j}^{\prime}$ (up) $\cup \mathfrak{O C E} \mathfrak{E}_{j+1}^{\prime}$ (up) has length at most 2. Let $\alpha_{1}^{\prime}=\left(r_{1}, c_{1}\right)>\alpha_{2}^{\prime}=\left(r_{2}, c_{2}\right)$ be such a $v$-chain. It follows from the proof of Corollary 4.14 (2) of [7] that $\alpha_{1}^{\prime} \in \mathfrak{O} \mathfrak{E}_{j}^{\prime}$ (up) and $\alpha_{2}^{\prime} \in \mathfrak{O} \mathfrak{E}_{j+1}^{\prime}$ (up). By item (1) of Lemma 5.3.2, it is enough to rule out the following possibility: $\alpha_{1}^{\prime}$ is of type $\mathrm{H}$ in $\alpha_{1}^{\prime}>\alpha_{2}^{\prime}$ and $p_{h}\left(\alpha_{1}^{\prime}\right)>\alpha_{2}^{\prime}$.

Suppose that this is the case. By Proposition 6.5.2 (1) and (2), it follows that there exist elements $\alpha_{1}=\left(r_{1}, C_{1}\right) \in \mathfrak{O} \mathfrak{E}_{j, j+1}^{\mathrm{pr}}$ and $\alpha_{2}=\left(R_{2}, c_{2}\right) \in \mathfrak{O E} \mathfrak{E}_{j+1}$ (up) with $C_{1} \leq$ $c_{1}$ and $r_{2} \leq R_{2}$. Since $p_{h}\left(\alpha_{1}^{\prime}\right)>\alpha_{2}^{\prime}$, it follows that $\alpha_{1}>\alpha_{2}$. Now, if $\alpha_{2}=p_{h}\left(\sigma_{j}\right)$, then Proposition 6.3.2 (2) is contradicted; if $\alpha_{2}$ belongs to $\mathfrak{O} \mathfrak{E}_{j+1}^{\mathrm{pr}}$, Proposition 6.3.2 (4) is contradicted (because $\left.p_{h}\left(\alpha_{1}\right)>\alpha_{2}\right)$.

\section{The map $\mathfrak{O} \phi$}

This section describes the map $\mathfrak{O} \phi$ and proves some basic facts about it. Certain proofs here refer to results from $\S 8$, but there is no circularity. As in $\S 6, j$ denotes an odd integer throughout.

\subsection{Description of $\mathfrak{O} \phi$}

The map $\mathfrak{O} \phi$ takes as input a pair $(w, \mathfrak{T})$, where $\mathfrak{T}$ is a monomial, possibly empty, in $\mathfrak{O N}(v)$ and $w \geq v$ an element of $\mathfrak{O} I(d)$ that $\mathfrak{O}$-dominates $\mathfrak{T}$, and produces as 
output a monomial $\mathfrak{O} \phi(w, \mathfrak{T})$ of $\mathfrak{O N}(v)$. To describe $\mathfrak{O} \phi$, we first partition $\mathfrak{T}$ into subsets $\mathfrak{D T}_{w, j, j+1}$, the partition being dependent on $w$.

Let $\mathfrak{S}_{w}^{j}$ (respectively $\mathfrak{S}_{w, j, j+1}$ ) denote the subset of $\mathfrak{S}_{w}$ consisting of those elements that are $j$-deep (respectively that are $j$ deep but not $j+2$ deep, or equivalently of depth $j$ or $j+1$ ) in $\mathfrak{S}_{w}$ in the sense of [7, §4]. Since $\mathfrak{S}_{w}$ is distinguished, symmetric, and has evenly many elements on the diagonal $\mathfrak{d}(v)$, it follows that $\mathfrak{S}_{w}^{j}$ and $\mathfrak{S}_{w, j, j+1}$ too have these properties, and that, in fact, the number of diagonal elements of $\mathfrak{S}_{w, j, j+1}$ is either 0 or 2 (in the latter case, the elements have to be distinct since $\mathfrak{S}_{w}$ is distinguished and so is multiplicity free). Let us denote by $w^{j}$ and $w_{j, j+1}$ the elements of $\mathfrak{O} I(d)$ corresponding to $\mathfrak{S}_{w}^{j}$ and $\mathfrak{S}_{w, j, j+1}$ by Proposition 2.6.1.

Let $\mathfrak{D T} \mathfrak{T}_{w, j, j+1}$ denote the subset of $\mathfrak{T}$ consisting of those elements $\alpha$ such that

- every $v$-chain in $\mathfrak{T}$ with head $\alpha$ is $\mathfrak{O}$-dominated by $w^{j}$, and

- there exists a $v$-chain in $\mathfrak{T}$ with head $\alpha$ that is not $\mathfrak{O}$-dominated by $w^{j+2}$.

It is evident that the subsets $\mathfrak{D T}_{w, j, j+1}$ are disjoint (as $j$ varies over the odd integers) and that their union is all of $\mathfrak{T}$ (for $w=w^{1} \mathfrak{O}$-dominates all $v$-chains in $\mathfrak{T}$ by hypothesis and $\mathfrak{S}_{w}^{j}$ is empty for large $j$ and so $w^{j}=v$ ). In other words, the $\mathfrak{O} \mathfrak{T}_{w, j, j+1}$ form a partition of $\mathfrak{T}$.

\section{Lemma 7.1.1}

(1) The length of a $v$-chain in $\mathfrak{O T} \mathfrak{T}_{w, j, j+1} \cup \mathfrak{D} \mathfrak{T}_{w, j, j+1}^{\#}$ is at most 2. In fact, the $\mathfrak{O}$ depth of any element in $\mathfrak{O T}_{w, j, j+1}$ is at most 2 .

(2) $w_{j, j+1} \mathfrak{O}$-dominates $\mathfrak{D T}_{w, j, j+1}$.

Proof The lemma follows rather easily from Corollary 8.2.3 as we now show. Let $C$ be a $v$-chain in $\mathfrak{D T}_{w, j, j+1}$. Let $\tau$ be the tail of $C$. Choose a $v$-chain $D$ in $\mathfrak{T}$ with head $\tau$ that is not $\mathfrak{O}$-dominated by $w^{j+2}$. Let $E$ be the concatenation of $C$ with $D$. Since the head of $E$ belongs to $\mathfrak{D T} \mathfrak{T}_{w, j, j+1}$, it follows that $E$ is $\mathfrak{O}$-dominated by $w^{j}$. It follows from (the only if part of) Corollary 8.2.3 (applied with $\mathfrak{E}=E$ and $x=w^{j}$ ) that $w_{j, j+1} \mathfrak{O}$-dominates $\mathfrak{O} E_{1}^{\mathrm{pr}} \cup \mathfrak{O} E_{2}^{\mathrm{pr}}$ and $w^{j+2} \mathfrak{O}$-dominates $\mathfrak{O} E^{3, p r}$. This means $\tau \notin \mathfrak{O} E^{3, p r}$, so $\tau \in \mathfrak{O} E_{1}^{\mathrm{pr}} \cup \mathfrak{O} E_{2}^{\mathrm{pr}}$, and so $C \subseteq \mathfrak{O} E_{1}^{\mathrm{pr}} \cup \mathfrak{O} E_{2}^{\mathrm{pr}}$. This proves (2). By Proposition 5.2.1 (2), the $\mathfrak{O}$-depths of elements of $C$ are the same in $C$ and $E$, so $C \subseteq \mathfrak{O} C_{1}^{\mathrm{pr}} \cup \mathfrak{O} C_{2}^{\mathrm{pr}}$, which proves the second assertion of (1). The first assertion of (1) follows from the second (see Lemma 5.3.1).

Corollary 7.1.2 $w_{j, j+1}$ dominates $\mathfrak{D T} \mathfrak{T}_{w, j, j+1} \cup \mathfrak{O T} \mathfrak{T}_{w, j, j+1}^{\#}$ in the sense of [7].

Proof Lemma 7.1.1 (2), Corollary 5.1.6 (taking $\mathfrak{E}=\mathfrak{O T} \mathfrak{T}_{w, j, j+1}$ and $w=w_{j, j+1}$ ).

So we may apply the map $\phi$ of $[7, \S 4]$ to the pair $\left(w_{j, j+1}, \mathfrak{O T} \mathfrak{T}_{w, j, j+1} \cup\right.$ $\left.\mathfrak{D T} \mathfrak{T}_{w, j, j+1}^{\#}\right)$ to obtain a monomial $\left(\mathfrak{D T} \mathfrak{T}_{w, j, j+1} \cup \mathfrak{D} \mathfrak{T}_{w, j, j+1}^{\#}\right)^{\star}$ in $\mathfrak{N}(v)$. In applying $\phi$, there is the partitioning of $\mathfrak{O} \mathfrak{T}_{w, j, j+1} \cup \mathfrak{O} \mathfrak{T}_{w, j, j+1}^{\#}$ into 'pieces', these being indexed by elements of $\mathfrak{S}_{w_{j, j+1}}=\mathfrak{S}_{w, j, j+1}$-observe that the elements of depth 1 (respectively 2) of $\mathfrak{S}_{w, j, j+1}$ are precisely those of $\mathfrak{S}_{w}$ of depth $j$ (respectively $j+1$ ). We denote by $\mathfrak{D P}_{\beta}$ the piece of $\mathfrak{D T} \mathfrak{T}_{w, j, j+1} \cup \mathfrak{O T} \mathfrak{T}_{w, j, j+1}^{\#}$ corresponding to $\beta$ in $\mathfrak{E}_{w j, j+1}$. 
We also use the notation $\mathfrak{O P}_{\beta}^{\star}$ where the 'star' operation on a piece is as defined in [7]. Moreover, we will use the phrase $\mathfrak{D}$-piece of $\mathfrak{T}$ (with respect to $w$ being implicitly understood) to refer to a piece of $\mathfrak{O T} \mathfrak{T}_{w, j, j+1} \cup \mathfrak{O T} \mathfrak{T}_{w, j, j+1}^{\#}$.

\section{Lemma 7.1.3}

1. The monomial $\left(\mathfrak{O T} \mathfrak{T}_{w, j, j+1} \cup \mathfrak{O} \mathfrak{T}_{w, j, j+1}^{\#}\right)^{\star}$ is symmetric and has either none or two distinct diagonal elements depending exactly on whether $\mathfrak{S}_{w_{j, j+1}}=\mathfrak{S}_{w, j, j+1}$ has 0 or 2 elements on the diagonal.

2. The depth of $\left(\mathfrak{D T} \mathfrak{T}_{w, j, j+1} \cup \mathfrak{O T}_{w, j, j+1}^{\#}\right)^{\star}$ is 2 ; the sets $\cup_{\beta \in\left(\mathfrak{S}_{w}\right)_{j}} \mathfrak{D P}_{\beta}^{\star}$ and $\cup_{\beta \in\left(\mathfrak{S}_{w}\right)_{j+1}} \mathfrak{O P}_{\beta}^{\star}$ are respectively those of the elements of depth 1 and 2 in $\left(\mathfrak{D T} \mathfrak{T}_{w, j, j+1} \cup \mathfrak{O} \mathfrak{T}_{w, j, j+1}^{\#}\right)^{\star}$.

Proof (1) The symmetry follows by combining Proposition 5.6 of [4], which says that the map $\pi$ respects the involution \#, with Proposition 4.2 of [7], which says that $\pi$ and $\phi$ are are inverses of each other.

The assertion about diagonal elements follows by combining item (B) of [4, Proposition 5.10], which is an assertion about the existence and relative multiplicities of diagonal elements in $\mathfrak{B}$ and $\mathfrak{B}^{\prime}$ where $\mathfrak{B}$ is a diagonal block of a monomial in $\mathfrak{N}(v)$, and Proposition 4.2 of [7].

(2) It follows from Propositions 4.2 of [7] that the map $\pi$ (described in $\S 4$ of that paper) applied to $\left(\mathfrak{D T} \mathfrak{T}_{w, j, j+1} \cup \mathfrak{O T} \mathfrak{T}_{w, j, j+1}^{\#}\right)^{\star}$ results in the pair $\left(w_{j, j+1}, \mathfrak{O T} \mathfrak{T}_{w, j, j+1} \cup\right.$

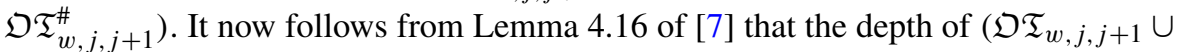
$\left.\mathfrak{D T} \mathfrak{T}_{w, j, j+1}^{\#}\right)^{\star}$ is exactly 2 . The latter assertions again follow from the results of [7]in fact, the proof that $\pi \circ \phi$ is identity on pages 47-49 of [7] shows that the $\mathfrak{O P}_{\beta}^{\star}$ are the blocks in the sense of [7] of the monomial $\left(\mathfrak{D T} w, j, j+1 \cup \mathfrak{D T} \mathfrak{T}_{w, j, j+1}^{\#}\right)^{\star}$.

Suppose that $\left(\mathfrak{D T} \mathfrak{T}_{w, j, j+1} \cup \mathfrak{D T} \mathfrak{T}_{w, j, j+1}^{\#}\right)^{\star}$ contains the pair $\left(a, a^{*}\right),\left(b, b^{*}\right)$ of diagonal elements with $a>b$. We call the pair $\left(b, a^{*}\right),\left(a, b^{*}\right)$ the twists, and set $\delta_{j}:=\left(b, a^{*}\right)$. In other words, $\delta_{j}$ is the element of the twisted pair that lies above the diagonal-observe that the twisted elements are reflections of each other. We allow ourselves the following ways of expressing the condition that $(\mathfrak{D T} w, j, j+1 \cup$ $\left.\mathfrak{O T} \mathfrak{T}_{w, j, j+1}^{\#}\right)^{\star}$ has diagonal elements: $\delta_{j}$ exists; $w$ is diagonal at $j$ (the latter expression is justified by the lemma above).

With notation as above, consider the new monomial defined as

$$
\begin{cases}(\mathfrak{D T} & \text { if } w \text { is not diagonal at } j \\ \left(\left(\mathfrak{D T} \mathfrak{T}_{w, j, j+1} \cup \mathfrak{D T} \mathfrak{T}_{w, j, j+1}^{\#}\right)^{\star} \backslash \mathfrak{d}(v)\right) \cup\left\{\delta_{j}, \delta_{j}^{\#}\right\} & \text { if } w \text { is diagonal at } j\end{cases}
$$

This new monomial is symmetric and contains no diagonal elements. Its intersection with $\mathfrak{O N}(v)$ is denoted $\mathfrak{O T} \mathfrak{T}_{w, j, j+1}^{\star}$. In other words, $\mathfrak{D} \mathfrak{T}_{w, j, j+1}^{\star}$ is the intersection of the new monomial with the subset of $\mathfrak{N}(v)$ of those elements that lie strictly above the diagonal.

The union of $\mathfrak{O} \mathfrak{T}_{w, j, j+1}^{\star}$ over all odd integers $j$ is defined to be $\mathfrak{O} \phi(w, \mathfrak{T})$. This finishes the description of the map $\mathfrak{O} \phi$. 
Fig. 9 To illustrate $\mathfrak{O} \phi$ : see Example 7.1.4

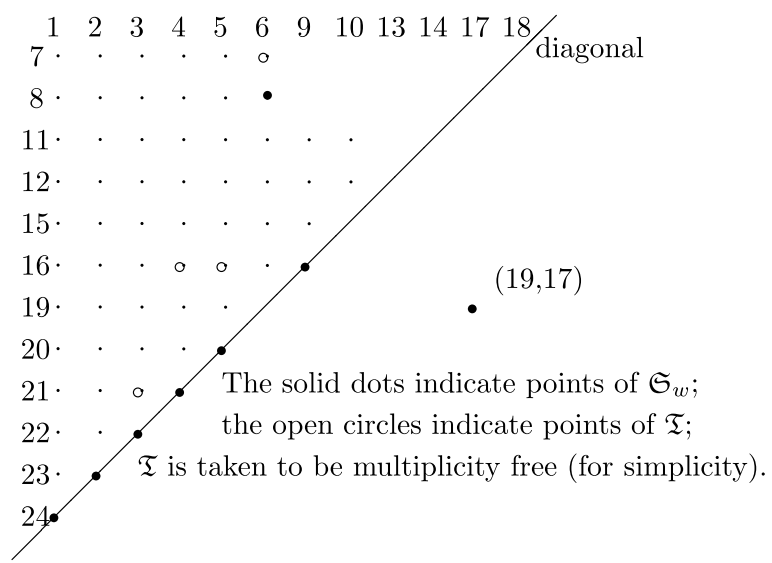

For $\beta$ in $\mathfrak{S}_{w, j, j+1}$ (up), we define the orthogonal piece-star $\mathfrak{O}(\mathfrak{T}, w, \beta)^{\star}$ corresponding to $\beta$ as

$$
\mathfrak{O}(\mathfrak{T}, w, \beta)^{\star}:= \begin{cases}\mathfrak{O P}_{\beta}^{\star}=\mathfrak{O P}_{\beta}^{\star}(\text { up }) & \text { if } \beta \text { is not on the diagonal } \\ \mathfrak{O P}_{\beta}^{\star} \cap \mathfrak{O N}(v) & \text { if } \beta \in\left(\mathfrak{S}_{w}\right)_{j+1} \text { is on the diagonal } \\ \left\{\mathfrak{O P} \mathfrak{P}_{\beta}^{\star} \cap \mathfrak{O N}(v)\right\} \cup\left\{\delta_{j}\right\} & \text { if } \beta \in\left(\mathfrak{S}_{w}\right)_{j} \text { is on the diagonal }\end{cases}
$$

With this, we can say that $\mathfrak{O} \phi(w, \mathfrak{T})$ is the union of $\mathfrak{O}(\mathfrak{T}, w, \beta)^{\star}$ as $\beta$ varies over $\mathfrak{S}_{w}$ (up).

Example 7.1.4 We illustrate by means of an example the map $\mathfrak{O} \phi$. Take $d=12, v=$ $(1,2,3,4,5,6,9,10,13,14,17,18), w=(8,10,13,14,16,18,19,20,21,22,23,24)$ and $\mathfrak{T}=\{(21,3),(16,4),(16,5),(7,6)\}$. Figure 9 shows the elements of $\mathfrak{S}_{w}$ and $\mathfrak{T}$.

We have:

$$
\begin{gathered}
w^{1}=w=(8,10,13,14,16,18,19,20,21,22,23,24), \\
w^{3}=(1,2,8,10,13,14,16,18,19,20,21,22), \\
w^{5}=(1,, 3,4,8,10,13,14,16,18,19,20) ; \\
\mathfrak{O T}_{w, 1,2} \text { is empty, } \quad \mathfrak{O T}_{w, 3,4}=\{(21,3)(16,4)\}, \\
\mathfrak{O T}_{w, 5,6}=\{(16,5)(7,6)\}, \quad \mathfrak{O T}_{w, j, j+1} \text { is empty for } j \geq 7 .
\end{gathered}
$$

Figure 10 shows the break up of $\mathfrak{T}$ into the $\mathfrak{D} \mathfrak{T}_{w, j, j+1}$.

Now:

$$
\begin{aligned}
& w_{1,2}=(3,4,5,6,9,10,13,14,17,18,23,24), \\
& w_{3,4}=(1,2,5,6,9,10,13,14,17,18,21,22), \\
& w_{5,6}=(1,2,3,4,8,10,13,14,16,18,19,20) .
\end{aligned}
$$


Fig. 10 To illustrate $\mathfrak{O} \phi$ : see Example 7.1.4

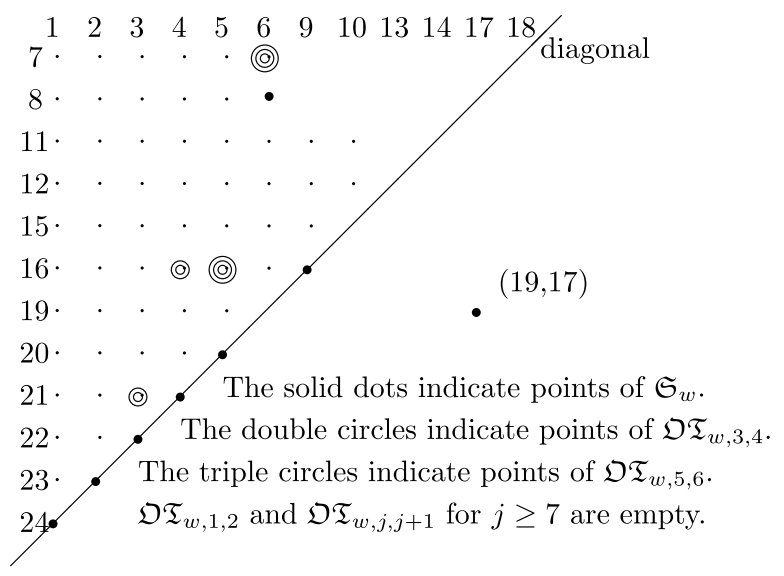

Fig. 11 To illustrate $\mathfrak{O} \phi$ : see Example 7.1.4. The single, double, and triple circles indicate respectively points of $\left(\mathfrak{D T}_{w, 1,2} \cup \mathfrak{O T}_{w, 1,2}\right)^{*}$, $\left(\mathfrak{O T} \mathfrak{T}_{w, 3,4} \cup \mathfrak{O T}_{w, 3,4}{ }^{\#}\right)^{*}$, and $\left(\mathfrak{O T} \mathfrak{T}_{w, 5,6} \cup \mathfrak{D T}_{w, 5,6}{ }^{\#}\right)^{*}$

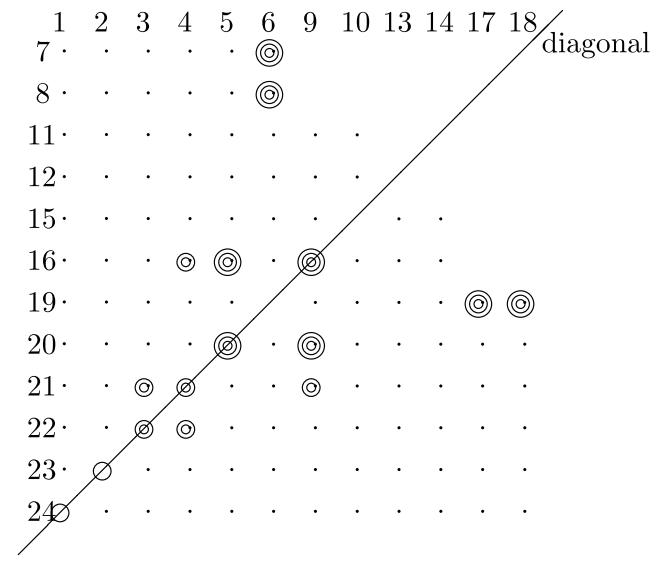

Applying the map $\phi$ of [7] to the pairs $\left(w_{j, j+1}, \mathfrak{D T} \mathfrak{T}_{w, 1,2} \cup \mathfrak{O T} \mathfrak{T}_{w, 1,2}^{\#}\right)$, we get:

$$
\begin{aligned}
& \left(\mathfrak{O T} \mathfrak{T}_{w, 1,2} \cup \mathfrak{O T} \mathfrak{T}_{w, 1,2}\right)^{*}=\{(24,1),(23,2)\}, \\
& \left(\mathfrak{D T} \mathfrak{T}_{w, 3,4} \cup \mathfrak{O T}_{w, 3,4}\right)^{*}=\{(21,3),(22,3),(22,4),(16,4),(21,4),(21,9)\} \text {, } \\
& \left(\mathfrak{D T} \mathfrak{T}_{w, 5,6} \cup \mathfrak{O T}_{w, 5,6}^{\#}\right)^{*}=\{(16,5),(20,5),(20,9),(16,9) \text {, } \\
& (7,6),(8,6),(19,17),(19,18)\}
\end{aligned}
$$

Figure 11 shows these monomials.

Clearly $w$ is diagonal at $1,3,5$; in other words $\delta_{1}, \delta_{3}, \delta_{5}$ exist: $\delta_{1}=(23,1), \delta_{3}=$ $(21,3)$, and $\delta_{5}=(16,5)$. We have:

$$
\begin{gathered}
\mathfrak{O T}_{w, 1,2}^{*}=\{(23,1)\}, \quad \mathfrak{O T}_{w, 3,4}^{*}=\{(21,3),(21,3),(16,4)\} \\
\mathfrak{D T}_{w, 5,6}^{*}=\{(16,5),(16,5),(7,6),(8,6)\} .
\end{gathered}
$$

Figure 12 shows these monomials. And, finally, Figure 12 shows the union of these monomials, which is $\mathfrak{O} \phi(w, \mathfrak{T})$. 
Fig. 12 To illustrate $\mathfrak{O} \phi$ : see Example 7.1.4

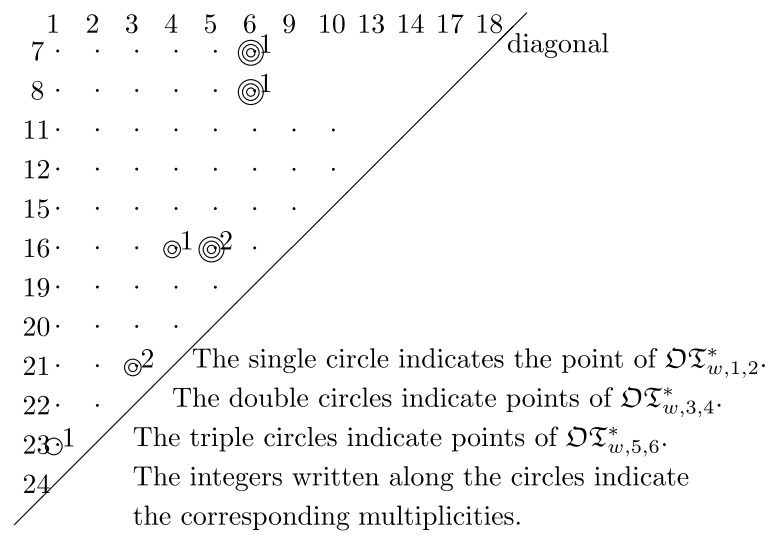

Lemma 7.1.5 Suppose that $\left(\mathfrak{O T}_{w, j, j+1} \cup \mathfrak{O T}_{w, j, j+1}^{\#}\right)^{\star}$ contains the pair $\left(a, a^{*}\right)$, $\left(b, b^{*}\right)$ of diagonal elements with $a>b$. Let

$$
\ldots,\left(r_{1}, c_{1}\right),\left(a, a^{*}\right),\left(c_{1}^{*}, r_{1}^{*}\right), \ldots ; \quad \ldots,\left(r_{2}, c_{2}\right),\left(b, b^{*}\right),\left(c_{2}^{*}, r_{2}^{*}\right), \ldots
$$

be respectively the elements of depth 1 and 2 of $\left(\mathfrak{O T} \mathfrak{T}_{w, j, j+1} \cup \mathfrak{O T} \mathfrak{T}_{w, j, j+1}^{\#}\right)^{\star}$ arranged in increasing order of row and column indices. Then

(1) $c_{1} \leq a^{*}$ and $r_{1} \leq b$ (assuming $\left(r_{1}, c_{1}\right)$ exists); and

(2) $r_{2}<b$ and $c_{2} \leq b^{*}$ (assuming $\left(r_{2}, c_{2}\right)$ exists).

Proof (1) Suppose that $\left(r_{1}, c_{1}\right)$ exists. It is clear that $c_{1} \leq a^{*}$. From way the map $\phi$ of [7] is defined, it follows that $\left(r_{1}, a^{*}\right)$ is an element of $\mathfrak{O T} \mathfrak{T}_{w, j, j+1}$. Suppose that $r_{1}>b$. Then $p_{h}\left(r_{1}, a^{*}\right)=\left(r_{1}, r_{1}^{*}\right)$ belongs to $\mathfrak{N}(v)$. We consider two cases.

If $\left(r_{2}, c_{2}\right)$ exists, then, again from the definition of the map $\phi$, it follows that $\left(r_{2}, b^{*}\right)$ is an element of $\mathfrak{O T} \mathfrak{T}_{w, j, j+1}$. But then $p_{h}\left(r_{1}, a^{*}\right)=\left(r_{1}, r_{1}^{*}\right)>\left(b, b^{*}\right)$ and $\left(b, b^{*}\right)$ dominates $\left(r_{2}, b^{*}\right)$, which means that the $v$-chain $\left(r_{1}, a^{*}\right)>\left(r_{2}, b^{*}\right)$ (note that $a^{*}<b^{*}$ because $a>b$ by hypothesis) in $\mathfrak{D T} w, j, j+1$ has $\mathfrak{O}$-depth more than 2 , a contradiction to Lemma 7.1.1 (1).

Now suppose that $\left(r_{2}, c_{2}\right)$ does not exist. (Then $\left(b, b^{*}\right)$ is the diagonal element in $\left(\mathfrak{S}_{w}\right)_{j+1}$.) Consider the singleton $v$-chain $C:=\left\{\left(r_{1}, a^{*}\right)\right\}$ in $\mathfrak{D T} w, j, j+1$. Then $\mathfrak{O m o n}_{C}=\left\{\left(a, a^{*}\right),\left(r_{1}, r_{1}^{*}\right)\right\}$ which is not dominated by $w_{j, j+1}$, a contradiction to Lemma 7.1.1 (2).

(2) Suppose that $\left(r_{2}, c_{2}\right)$ exists. Then there exists, by the definition of the map $\phi$, an element $\left(r_{2}, b^{*}\right)$ in $\mathfrak{D T} w, j, j+1$. Since $\left(r_{2}, b^{*}\right)$ lies above the diagonal, it follows that $r_{2}<b$. That $c_{2} \leq b^{*}$ is clear.

7.2 Basic facts about $\mathfrak{D T} \mathfrak{T}_{w, j, j+1}$ and $\mathfrak{D T} \mathfrak{T}_{w, j, j+1}^{\star}$

\section{Lemma 7.2.1}

(1) Let $\alpha^{\prime}>\alpha$ be elements of $\mathfrak{T}$. Let $j$ and $j^{\prime}$ be the odd integers such that $\alpha^{\prime} \in$ $\mathfrak{T}_{w, j^{\prime}, j^{\prime}+1}$ and $\alpha \in \mathfrak{O} \mathfrak{T}_{w, j, j+1}$. Then $j^{\prime} \leq j$. 
(2) If, further, either

(a) there exists $\mu$ in $\mathfrak{T}$ such that $\alpha^{\prime}>\mu>\alpha$, or

(b) $\alpha^{\prime} \in \mathfrak{O P}_{\beta^{\prime}}$ for $\beta^{\prime}$ in $\left(\mathfrak{E}_{w}\right)_{j^{\prime}+1}$,

then $j^{\prime}<j$.

Proof (1) By hypothesis, every $v$-chain with head $\alpha^{\prime}$ is $\mathfrak{O}$-dominated by $w^{j^{\prime}}$. This implies, by Corollary 5.2.2, that every $v$-chain with head $\alpha$ is $\mathfrak{O}$-dominated by $w^{j^{\prime}}$. This shows $j^{\prime} \leq j$.

(2a) Suppose that $j^{\prime}=j$. It follows from (1) that $\alpha^{\prime}, \mu$, and $\alpha$ all belong to $\mathfrak{D T} \mathfrak{T}_{w, j, j+1}$. But then $\alpha^{\prime}>\mu>\alpha$ is a $v$-chain of length 3 in $\mathfrak{D T}_{w, j, j+1}$, a contradiction to Lemma 7.1.1 (1).

(2b) Suppose that $j^{\prime}=j$. Then $\alpha^{\prime}>\alpha$ is a $v$-chain in $\mathfrak{O T}_{w, j, j+1}$. Being of length 2 , it cannot be dominated by $\left(\mathfrak{E}_{w}\right)_{j+1}$, which means, by the definition of $\mathfrak{O P} \beta_{\beta^{\prime}}$, that $\alpha^{\prime}$ cannot belong to $\mathfrak{O P}_{\beta^{\prime}}$, a contradiction.

\section{Proposition 7.2.2}

(1) The length of a $v$-chain in $\mathfrak{D T}_{w, j, j+1}^{\star}$ is at most 2.

(2) The $\mathfrak{D}$-depth of $\mathfrak{O} \mathfrak{T}_{w, j, j+1}^{\star}$ is at most 2.

(3) $\cup_{\beta \in\left(\mathfrak{S}_{w}\right)_{j}(\mathrm{up})} \mathfrak{O}(\mathfrak{T}, w, \beta)^{\star}$ is the set of depth 1 elements of $\mathfrak{D} \mathfrak{T}_{w, j, j+1}^{\star}$ (in particular, no two elements there are comparable); if $\delta_{j}$ exists, then it is the last element of $\cup_{\beta \in\left(\mathfrak{S}_{w}\right)_{j} \text { (up) }} \mathfrak{O}(\mathfrak{T}, w, \beta)^{\star}$ when the elements are arranged in increasing order of row and column indices.

(4) $\cup_{\beta \in\left(\mathfrak{S}_{w}\right)_{j+1}(\mathrm{up})} \mathfrak{O}(\mathfrak{T}, w, \beta)^{\star}$ is the set of depth 2 elements of $\mathfrak{O T}_{w, j, j+1}^{\star}$ (in particular, no two elements there are comparable); if $\delta_{j}$ exists, then its row index exceeds the row index of any element in $\cup_{\beta \in\left(\mathfrak{S}_{w}\right)_{j+1}(\mathrm{up})} \mathfrak{O}(\mathfrak{T}, w, \beta)^{\star}$.

Proof For (1), it is enough, given Lemma 7.1.3 (2), to show that $\delta_{j}$ is not comparable to any element of depth 1 of $\left(\mathfrak{D T} \mathfrak{T}_{w, j, j+1} \cup \mathfrak{O T} \mathfrak{T}_{w, j, j+1}^{\#}\right)^{\star}$, and this follows from Lemma 7.1.5 (1). In fact, the above argument proves also (3).

For (4), it is enough, given Lemma 7.1.3 (2), the symmetry of the monomials involved in that lemma, and the observation that $\alpha>\beta$ implies $\alpha$ (up) $>\beta$ (up) for elements $\alpha, \beta$ of $\mathfrak{N}(v)$, to show the following: if $\left(a, a^{*}\right)>\gamma=(e, f)$ for $\gamma$ an element of $\left(\mathfrak{D T} \mathfrak{T}_{w, j, j+1} \cup \mathfrak{O T} \mathfrak{T}_{w, j, j+1}^{\#}\right)^{\star}$ lying (strictly) above the diagonal, then $\delta_{j}>\gamma$. But this follows from Lemma 7.1.5 (2): $\gamma$ is a depth 2 element in $\left(\mathfrak{D T}_{w, j, j+1} \cup\right.$ $\left.\mathfrak{O T} \mathfrak{T}_{w, j, j+1}^{\#}\right)^{\star}$, and we have $e \leq r_{2}<b$ (and $a^{*}<f$ since $\left.\left(a, a^{*}\right)>\gamma\right)$. In fact, the above argument proves also (2): observe that $f \leq b^{*}$ (Lemma 7.1.5 (2)).

\section{Some lemmas}

This section is preparatory towards the proof in $\$ 9$ of the main combinatorial results of this paper, namely Propositions 4.3.1 and 4.3.2. These being analogues of Propositions 4.1 and 4.2 of [7], their proofs should naturally be analogous too. The proofs in [7] rely on certain lemmas and it is natural therefore to first establish the orthogonal analogues of those. The purpose of this section is precisely that. 
The lemmas below, especially those in $\S 8.4$, may be unintelligible until one tries to read $\S 9$. The division of this section into four subsections is also suggested by the structure of the proofs in [7].

\subsection{Lemmas from the Grassmannian case}

In this subsection, the terminology and notation of [7, §4] are in force. The statements here, while they do not explicitly appear in [7, \$4], are but slightly more precise versions of ones that do appear there, and so we omit the proofs (which in any case can be found in $[20, \S 9.1])$.

For a monomial $\mathfrak{F}$ in $\mathfrak{N}(v)$ and $k$ a positive integer, denote by $\mathfrak{F}_{k}$ the set of elements of depth $k$ of $\mathfrak{F}$ (as in [7]), by $\mathfrak{F}^{k}$ the set of elements of depth at least $k$ of $\mathfrak{F}$, and set $\mathfrak{F}_{k, k+1}:=\mathfrak{F}_{k} \cup \mathfrak{F}_{k+1}$.

Lemma 8.1.1 Let $\mathfrak{F}$ be a monomial in $\mathfrak{N}(v)$, and let $\pi(\mathfrak{F})=\left(w, \mathfrak{F}^{\prime}\right)$, where $\pi$ is the map defined in [7, \$4]. Then the maximum length of a $v$-chain in $\mathfrak{F} \cup \mathfrak{F}^{\prime}$ is the same as the maximum length of a $v$-chain in $\mathfrak{F}$.

Lemma 8.1.2 Let $\mathfrak{B}$ and $\mathfrak{U}$ be monomials in $\mathfrak{N}(v)$. Assume that

- the elements of $\mathfrak{B}$ form a single block (in the sense of [7, Page 38]).

- $\mathfrak{U}$ has depth 1 (equivalently, there are no comparable elements in $\mathfrak{U}$ ).

- for every $\beta=(r, c)$ in $\mathfrak{B}$, there exist $\gamma^{1}(\beta)=\left(R^{1}, C^{1}\right)$, and $\gamma^{2}(\beta)=\left(R^{2}, C^{2}\right)$ in $\mathfrak{U}$ such that

$$
C^{1}<c, \quad C^{2}<R^{1}, \quad r<R^{2}
$$

this holds, for example, when there exists $\gamma(\beta)$ in $\mathfrak{U}$ such that $\gamma(\beta)>\beta$ : take $\gamma^{1}(\beta)=\gamma^{2}(\beta)=\gamma(\beta)$.

Then there exists a unique block $\mathfrak{C}$ of $\mathfrak{U}$ such that $w(\mathfrak{C})>w(\mathfrak{B})$.

Let $x$ be an element of $I(d, n)$ and $\mathfrak{S}_{x}$ the corresponding distinguished subset in $\mathfrak{N}(v)$ (see $\$ 2.6 .2)$. For $k$ a positive integer, let $x_{k}, x_{k, k+1}, x^{k}$ denote respectively the elements of $I(d, n)$ corresponding to the distinguished subsets $\left(\mathfrak{S}_{x}\right)_{k},\left(\mathfrak{S}_{x}\right)_{k, k+1}$, $\left(\mathfrak{S}_{x}\right)^{k}$. As a corollary of [7, Lemma 4.5] we have:

Lemma 8.1.3 Let $\mathfrak{F}$ be a monomial in $\mathfrak{N}(v)$ and $x$ an element of $I(d, n)$. For $x$ to dominate $\mathfrak{F}$ it is necessary and sufficient that for every $\alpha=(r, c)$ in $\mathfrak{F}$ there exist $\beta=(R, C)$ in $\mathfrak{S}_{x}$ with $C \leq c, r \leq R$ and $\operatorname{depth}_{\mathfrak{S}_{x}} \beta \geq \operatorname{depth}_{\mathfrak{F}} \alpha$.

Corollary 8.1.4 $x$ dominates $\mathfrak{F} \Leftrightarrow x_{k}$ dominates $\mathfrak{F}_{k} \forall k \Leftrightarrow x_{1,2}$ dominates $\mathfrak{F}_{1,2}$ and $x^{3}$ dominates $\mathfrak{F}^{3}$.

\subsection{Orthogonal analogues of lemmas of 8.1}

Lemma 8.2.2 below is the orthogonal analogue of Lemma 8.1.3 (more precisely, that of the first assertion of Corollary 8.1.4). The following proposition will be used in its proof. 
Proposition 8.2.1 Let $x$ be an element of $\mathfrak{O} I(d)$ and $\mathfrak{E}$ a monomial in $\mathfrak{O N}(v)$. Then $x \mathfrak{O}$-dominates $\mathfrak{O E}_{1}^{\mathrm{pr}} \cup \mathfrak{O} \mathfrak{E}_{2}^{\mathrm{pr}}$ if and only if it $\mathfrak{O}$-dominates every $v$-chain in $\mathfrak{E}$ of $\mathfrak{O}$-depth at most 2.

Proof The 'if' part is immediate from definitions (see also Proposition 6.5.4). For the 'only if' part, let $C$ be a $v$-chain in $\mathfrak{E}$ of $\mathfrak{O}$-depth at most 2 . To show that $x$ dominates $\mathfrak{O m o n}_{C}$, it is enough, by Corollary 8.1.4, to show that $x_{1}$ dominates $(\mathfrak{O m o n})_{C}$ and $x_{2}$ dominates $(\mathfrak{O m o n})_{2}$ (by choice of $C,(\mathfrak{D m o n})_{k}$ is empty for $k \geq 3$ ).

Let $\alpha^{\prime} \in\left(\mathfrak{O m o n}_{C}\right)_{1}$. Choose $\alpha$ in $C$ such that $\alpha^{\prime} \in \mathfrak{O m o n}_{C, \alpha}$. Choose $\alpha_{0}$ in $\mathfrak{O} \mathfrak{E}_{1}^{\mathrm{pr}}$ such that $\alpha_{0}$ dominates $\alpha$. Since $x \mathfrak{O}$-dominates the singleton $v$-chain $\left\{\alpha_{0}\right\}$, it follows that $x_{1}$ dominates $\mathfrak{O} q_{\left\{\alpha_{0}\right\}, \alpha_{0}}$. We claim that $\mathfrak{O} q_{\left\{\alpha_{0}\right\}, \alpha_{0}}$ dominates $\alpha^{\prime}$. To prove the claim, we need only rule out the possibility that $\alpha_{0}$ is of type $S$ in $\left\{\alpha_{0}\right\}$ and $\alpha$ of type $\mathrm{V}$ in $C$. Since $\alpha^{\prime} \in\left(\mathfrak{O m o n}_{C}\right)_{1}$, it follows from Proposition 5.1 .4 (1) that $\alpha$ is the first element of $C$. In particular, if $\alpha$ is of type V in $C$, then $p_{h}(\alpha) \in \mathfrak{N}(v)$, so $p_{h}\left(\alpha_{0}\right) \in \mathfrak{N}(v)$, and $\alpha_{0}$ is of type $\mathrm{H}$ in $\left\{\alpha_{0}\right\}$. The claim is thus proved.

Now consider an element of $\left(\mathfrak{O m o n}_{C}\right)_{2}$. Observe that the length of $C$ is at most 2 (Lemma 5.3.1). So our element is either the horizontal projection $p_{h}(\alpha)$ of the head $\alpha$ of $C$, or it is $\mathcal{O} q_{C, \beta}$ where $\beta$ is the tail of $C$. In the first case, let $\alpha_{0}$ be as in the previous paragraph, and proceed similarly. It is clear that $p_{h}\left(\alpha_{0}\right) \in \mathfrak{N}(v)$ (because $\left.p_{h}(\alpha) \in \mathfrak{N}(v)\right) ; x_{2}$ dominates $p_{h}\left(\alpha_{0}\right)$ and so also $p_{h}(\alpha)$.

Now we handle the second case. If $\beta \in \mathfrak{O} \mathfrak{E}_{2}^{\mathrm{pr}}$, then $C$ is contained in $\mathfrak{O E}_{1}^{\mathrm{pr}} \cup \mathfrak{O} \mathfrak{E}_{2}^{\mathrm{pr}}$ and there is nothing to prove. So assume that $\mathfrak{O}$-depth $\operatorname{de}_{\mathfrak{E}}(\beta) \geq 3$. Choose a $v$-chain $D$ in $\mathfrak{E}$ with tail $\beta, \mathfrak{O}$-depth $\operatorname{dep}_{D}(\beta) \geq 3$, and with the good property as in Proposition 5.3.4. There occurs in $D$ an element of $\mathfrak{O}$-depth 3 , say $\delta$. (Lemma 5.3.2 (4)). Let $A$ denote the part $\delta>\ldots$ of $D$ and $C^{\prime}$ the part up to but not including $\delta$. There clearly is an element-call it $\mu$-of depth 2 in $\mathfrak{O m o n}_{D}$ that dominates $\mathfrak{O} q_{D, \beta}$. This element $\mu$ belongs to $\mathfrak{O m o n}_{C^{\prime}}$ (Corollary 5.3.6 (3)). Since $D$ has the good property of Proposition 5.3.4, $C^{\prime} \subseteq \mathfrak{O} \mathfrak{E}_{1}^{\mathrm{pr}} \cup \mathfrak{O} \mathfrak{E}_{2}^{\mathrm{pr}}$, so $\mu$ is dominated by an element in $\left(\mathfrak{S}_{x}\right)_{2}$. In particular, $\mathfrak{O} q_{D, \beta}$ is dominated by the same element of $\left(\mathfrak{S}_{x}\right)_{2}$.

We are still not done, for it is possible that $\mathcal{O} q_{D, \beta}$ be $\beta$ and $\mathfrak{O} q_{C, \beta}$ be $p_{v}(\beta)$. Suppose that this is the case. Then $\alpha>\beta$ is connected. So $p_{h}(\alpha) \in \mathfrak{N}(v)$ and the legs of $\alpha$ and $\beta$ intertwine. As seen above in the third paragraph of the present proof, there is an element of $\left(\mathfrak{S}_{x}\right)_{2}$ that dominates $p_{h}(\alpha)$. By the distinguishedness of $\mathfrak{S}_{x}$, it follows that the element in $\left(\mathfrak{S}_{x}\right)_{2}$ dominating $\beta$ is the same as the one dominating $p_{h}(\alpha)$. By the symmetry of $\mathfrak{S}_{x}$, this element lies on the diagonal and so dominates $p_{v}(\beta)$, and, finally, we are done with the proof in the second case.

As is easily seen, for $x \in \mathfrak{O} I(d)$ and $j$ an odd integer, $x_{j, j+1}$ and $x^{j}$ belong to $\mathfrak{O} I(d)$-see Proposition 2.6.1.

Lemma 8.2.2 Let $\mathfrak{E}$ be a monomial in $\mathfrak{O N}(v)$ and $x$ an element of $\mathfrak{O} I(d)$. For $x$ to $\mathfrak{O}$-dominate $\mathfrak{E}$ it is necessary and sufficient that, for every odd integer $j$, every $v$-chain in $\mathfrak{O} \mathfrak{E}_{j}^{\mathrm{pr}} \cup \mathfrak{O} \mathfrak{E}_{j+1}^{\mathrm{pr}}$ is $\mathfrak{O}$-dominated by $x_{j, j+1}$.

Proof First suppose that $x$ dominates $\mathfrak{E}$. Let $j$ be an odd integer and let $A$ a $v$-chain in $\mathfrak{O C E} \mathfrak{E}_{j}^{\mathrm{pr}} \cup \mathfrak{O} \mathfrak{E}_{j+1}^{\mathrm{pr}}$. We need to show that $x_{j, j+1}$ dominates $\mathfrak{O m o n} \mathfrak{m}_{A}$. For this, we may 
assume that $A$ is maximal (by Corollary 5.2.2). By Corollary 5.2.3 (3), the length of $A$ is at most 2. By Lemma 5.3.2 (4) (b), for every $\beta$ in $\mathfrak{O} \mathfrak{E}_{j+1}^{\mathrm{pr}}$ there exists $\alpha$ in $\mathfrak{O} \mathfrak{E}_{j}^{\mathrm{pr}}$ with $\alpha>\beta$. Thus we may assume that the head $\alpha$ of $A$ belongs to $\mathfrak{O} \mathfrak{E}_{j}^{\mathrm{pr}}$.

It is enough to show (see [7, Lemma 4.5]) that for any $v$-chain $E$ in $\mathfrak{O m o n} \mathfrak{m}_{A}$

- the length of $E$ is at most 2;

- there exists an $x$-dominated monomial in $\mathfrak{N}(v)$ containing $E$ and the head of $E$ is an element of depth at least $j$ in that monomial.

The first of these conditions holds by Proposition 6.5.4. We now show that the second holds.

We may assume that $E$ is maximal in $\mathfrak{O m o n}_{A}$. By Proposition 5.1.4 (1), the head of $E$ is $\mathfrak{O} q_{A, \alpha}$. Let $C$ a $v$-chain in $\mathfrak{E}$ with tail $\alpha$ such that $\mathfrak{O}$-depth ${ }_{C}(\alpha)=j$. Let $D$ be the concatenation of $C$ with $A$. We claim that the monomial $\mathfrak{O m o n} \mathfrak{n}_{D}$ has the desired properties. That $\mathfrak{O m o n}_{D}$ is $x$-dominated is clear (since $x \mathfrak{O}$-dominates $\mathfrak{E}$ ). By Corollary 5.3.6, it follows that $\mathfrak{O} q_{D, \alpha}=\mathfrak{O} q_{A, \alpha}$ and $\mathfrak{O m o n} \mathfrak{n}_{A} \subseteq \mathfrak{O m o n} \mathfrak{m}_{D}$ (in particular that $\left.E \subseteq \mathfrak{O m o n}_{D}\right)$. By Proposition 5.2.1 (2), $\mathfrak{O}-\operatorname{depth}_{D}(\alpha)=\mathfrak{O}-\operatorname{depth}_{C}(\alpha)=j$, that is, depth $\mathfrak{O m o n}_{D} \mathfrak{O} q_{D, \alpha}=j$. The proof of the necessity is thus complete.

To prove the sufficiency, proceed by induction on the largest odd integer $J$ such that $\mathfrak{O E} \mathfrak{E}_{J}^{\mathrm{pr}} \cup \mathfrak{O E} \mathfrak{E}_{J+1}^{\mathrm{pr}}$ is non-empty. When $J=1$, there is nothing to prove, for $\mathfrak{O} \mathfrak{E}_{1}^{\mathrm{pr}} \cup$ $\mathfrak{O E} \mathfrak{E}_{2}^{\mathrm{pr}}=\mathfrak{E}$ and $x_{1,2} \mathfrak{O}$-dominates $\mathfrak{O E}_{1}^{\mathrm{pr}} \cup \mathfrak{O} \mathfrak{E}_{2}^{\mathrm{pr}}$. So suppose that $J \geq 3$. We implicitly use Corollary 5.3.8 in what follows. By induction, $x^{3} \mathfrak{O}$-dominates $\mathfrak{O} \mathfrak{E}^{3,4}$.

Let $D$ be a $v$-chain in $\mathfrak{E}$. Our goal is to show that $x$ dominates $\mathfrak{O m o n}_{D}$. Let $\alpha$ be the element of $D$ with $\mathfrak{O}$-depth ${ }_{D}(\alpha)=3$ - such an element exists, by Lemma 5.3.2 (4) (if there exists in $D$ an element of $\mathfrak{O}$-depth in $D$ exceeding 2); the following proof works also in the case when $\alpha$ does not exist. Let $A$ be the part $\alpha>\ldots$ of $D$, and $C^{\prime}$ the part up to but not including $\alpha$. By Proposition 5.2.1 (2), the $\mathfrak{O}$-depth (in $C^{\prime}$ ) of elements of $C^{\prime}$ is at most 2. By Proposition 8.2.1, $x_{1,2}$ dominates $\mathfrak{O m o n} C^{\prime}$. By Corollary 5.3.6 (3), $\left(\mathfrak{O m o n}_{D}\right)_{1,2}=\mathfrak{O m o n}_{C^{\prime}}$ and $(\mathfrak{O m o n})_{D}^{3}=\mathfrak{O m o n}_{A}$. Since $A \subseteq \mathfrak{O} \mathfrak{E}^{3,4}$, it follows that $x^{3}$ dominates $\mathfrak{O m o n}_{A}$ (induction hypothesis). Finally, by an application of Corollary 8.1.4, we conclude that $x$ dominates $\mathfrak{O m o n} \mathfrak{m}_{D}$.

Corollary 8.2.3 An element $x$ of $\mathfrak{O} I(d) \mathfrak{O}$-dominates a monomial $\mathfrak{E}$ in $\mathfrak{O N}(v)$ if and only if $x_{1,2} \mathfrak{O}$-dominates $\mathfrak{O} \mathfrak{E}_{1}^{\mathrm{pr}} \cup \mathfrak{O} \mathfrak{E}_{2}^{\mathrm{pr}}$ and $x^{3} \mathfrak{O}$-dominates $\mathfrak{O} \mathfrak{E}^{3,4}$.

Proof It is easy to see that $\left(x^{3}\right)_{j, j+1}=x_{j+2, j+3}$; it follows from Proposition 5.3.7 that $\mathfrak{O}\left(\mathfrak{O E} \mathfrak{E}^{3,4}\right)_{j}^{\mathrm{pr}} \cup \mathfrak{O}\left(\mathfrak{O E} \mathfrak{E}^{3,4}\right)_{j+1}^{\mathrm{pr}}=\mathfrak{O} \mathfrak{E}_{j+2}^{\mathrm{pr}} \cup \mathfrak{O} \mathfrak{E}_{j+3}^{\mathrm{pr}}$. The assertion follows from the lemma.

\subsection{Orthogonal analogues of some lemmas in [7]}

The proofs of Propositions 4.1 and 4.2 of [7] are based on assertions 4.9-4.16 (of that paper). The orthogonal analogues of these assertions are proved in this section. Assertion 4.9 exactly as in [7] is relevant in the present context and used frequently below, so we recall it explicitly as Lemma 8.3.1.

Throughout this section $\mathfrak{E}$ denotes a monomial in $\mathfrak{O N}(v)$. An $\mathfrak{O}$-block of $\mathfrak{E}$ means a block in the sense of [7] of $\mathfrak{O \mathfrak { E } _ { k }}$ for some integer $k$. 
Lemma 8.3.1 If $\mathfrak{B}_{1}, \ldots, \mathfrak{B}_{l}$ are the blocks in order from left to right of some $\mathfrak{O E}_{k}$, and $w\left(\mathfrak{B}_{1}\right)=\left(R_{1}, C_{1}\right), w\left(\mathfrak{B}_{2}\right)=\left(R_{2}, C_{2}\right), \ldots, w\left(\mathfrak{B}_{l}\right)=\left(R_{l}, C_{l}\right)$, then

$$
C_{1}<R_{1}<C_{2}<R_{2}<\ldots<R_{l-1}<C_{l}<R_{l}
$$

Proof This is merely a recall Lemma 4.9 of [7]. In any case, it is easy.

Lemma 8.3.2 No two elements of $\mathfrak{O} \mathfrak{E}_{k}(\mathrm{ext}) \cup \mathfrak{O} \mathfrak{E}_{k}^{\prime}$ are comparable. More precisely, it is not possible to have elements $\alpha>\beta$ both belonging to $\mathfrak{D E} \mathfrak{E}_{k}(\mathrm{ext}) \cup \mathfrak{O \mathfrak { E } _ { k } ^ { \prime }}$.

Proof It follows from Lemma 8.1.1 that $\mathfrak{\mathcal { O E }} \mathfrak{E}_{k} \cup \mathfrak{O \mathfrak { E } _ { k } ^ { \prime }}$ contains no comparable elements. If $k$ is even, then $\mathfrak{O E}_{k}\left(\right.$ ext) $=\mathfrak{O} \mathfrak{E}_{k}$ (Corollary 6.3.4 (2)); if $k$ is odd, we may assume $\mathfrak{O E}_{k}($ ext $)=\mathfrak{O} \mathfrak{E}_{k}$ (as sets) by increasing the multiplicity of $\sigma_{k}$ in $\mathfrak{O} \mathfrak{E}_{k}^{\mathrm{pr}}$.

Lemma 8.3.3 For integers $i \leq k$, there cannot exist $\gamma \in \mathfrak{O E}_{i}^{\prime}(\mathrm{up})$ and $\beta \in \mathfrak{O} \mathfrak{E}_{k}^{\mathrm{pr}}$ such that $\beta>\gamma$. For integers $i<k$, there cannot exist $\gamma \in \mathfrak{O} \mathfrak{E}_{i}^{\prime}$ (up) and $\beta \in \mathfrak{O} \mathfrak{E}_{k}^{\mathrm{pr}}$ such that $\beta$ dominates $\gamma$.

Proof Let $\gamma \in \mathfrak{O} \mathfrak{E}_{i}^{\prime}$ (up) and $\beta \in \mathfrak{O} \mathfrak{E}_{k}^{\mathrm{pr}}$. If $i=k$ and $\beta>\gamma$, then we get a contradiction immediately to Lemma 8.3.2. Now suppose that $i<k$ and that $\beta$ dominates $\gamma$. Apply Corollary 5.3.5 (the notation of the corollary being suggestive of how exactly to apply it). Let $\alpha$ be as in its conclusion. The chain $\alpha>\gamma$ contradicts Lemma 8.3.2 in case $i$ is odd and either Lemma 8.3.2 or Proposition 6.5.4 in case $i$ is even.

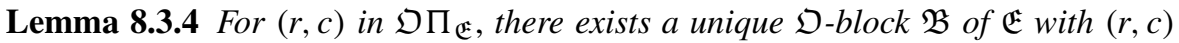
in $\mathfrak{B}^{\prime}$.

Proof The existence is clear from the definition of $\mathfrak{O} \Pi_{\mathfrak{E}}$. For the uniqueness, suppose that $\mathfrak{B}$ and $\mathfrak{C}$ are two distinct $\mathfrak{O}$-blocks of $\mathfrak{E}$ with $(r, c)$ in both $\mathfrak{B}^{\prime}$ and $\mathfrak{C}^{\prime}$. We will show that this leads to a contradiction.

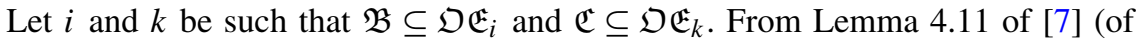
which the present lemma is the orthogonal analogue) it follows that $i \neq k$, so we can assume without loss of generality that $i<k$. By applying the involution \# if necessary, we may assume that $(r, c) \in \mathfrak{O} \mathfrak{E}_{i}^{\prime}$ (up). Now there exists an element $(r, a)$ in $\mathfrak{C}$ with $a \leq c$ (this follows from the definition of $\mathfrak{C}^{\prime}$ ). Clearly $(r, a) \in \mathfrak{O} \mathfrak{E}_{k}^{\mathrm{pr}}$. Taking $\beta=(r, a)$ and $\gamma=(r, c)$, we get a contradiction to Lemma 8.3.3.

Lemma 8.3.5 Let $i<j$ be positive integers.

(1) For a block $\mathfrak{B}$ of $\mathfrak{O} \mathfrak{E}_{j}$, there exists a unique block $\mathfrak{C}$ of $\mathfrak{O} \mathfrak{E}_{i}$ with $w(\mathfrak{C})>w(\mathfrak{B})$.

(2) For $\beta$ in $\mathfrak{O E} \mathfrak{E}_{j}(\mathrm{ext}) \cup \mathfrak{O} \mathfrak{E}_{j}^{\prime}$, there exists $\alpha$ in $\mathfrak{O} \mathfrak{E}_{i}$ such that $\alpha>\beta$.

Proof (1): The assertion follows by applying Lemma 8.1 .2 with $\mathfrak{B}=\mathfrak{B}$ and $\mathfrak{U}=$ $\mathfrak{O E} \mathfrak{E}_{i}$. We need to make sure however that the lemma can be applied. More precisely, we need to check that for every $\beta=(r, c)$ in $\mathfrak{B}$ there exist $\gamma^{1}(\beta)=\left(R^{1}, C^{1}\right)$ and $\gamma^{2}(\beta)=\left(R^{2}, C^{2}\right)$ in $\mathfrak{O E}_{i}$ such that $C^{1}<c, C^{2}<R^{1}$, and $r<R^{2}$. We may 
assume $\beta=\beta$ (up), for, if $\beta=\beta$ (down), then $\beta$ (up) also belongs to $\mathfrak{O E}_{j}$ because $\mathfrak{O E} \mathfrak{E}_{j}$ is symmetric, and we can set $\gamma^{1}(\beta)=\gamma^{2}\left(\beta\right.$ (up))(down), and $\gamma^{2}(\beta)=$ $\gamma^{1}(\beta$ up $)$ (down) - note that these two belong to $\mathfrak{O \mathfrak { E } _ { i }}$ since $\mathfrak{O} \mathfrak{E}_{i}$ is symmetric.

We consider three cases:

(1) $\beta$ belongs to $\mathfrak{E}$.

(2) $\beta=p_{h}\left(\sigma_{j-1}\right)$ (in particular, $j$ is even and $\mathfrak{E}$ is truly orthogonal at $j-1$ ).

(3) $\beta=p_{v}\left(\sigma_{j}\right)$ (in particular, $j$ is odd and $\mathfrak{E}$ is truly orthogonal at $j$ ).

Define $\beta^{\prime}$ to be $\beta$ in case $1, \sigma_{j-1}$ in case 2 , and $\sigma_{j}$ in case 3. Let $C$ be a $v$-chain in $\mathfrak{E}$ with tail $\beta^{\prime}$ and having the good property as in Proposition 5.3.4.

First suppose that there exists in $C$ an element of $\mathfrak{O}$-depth $i$ and denote it by $\gamma$. If $p_{h}(\gamma) \notin \mathfrak{N}(v)$ (this can happen only in case 1), then set $\gamma^{1}(\beta)=\gamma^{2}(\beta)=\gamma$. Now suppose $p_{h}(\gamma) \in \mathfrak{N}(v)$. Then $\gamma \in \mathfrak{O E}_{i}$ except when $\gamma=\sigma_{i}$ with $i$ odd and $\sigma_{i}$ has multiplicity 1 in $\mathfrak{E}$. If $\gamma \in \mathfrak{O E}_{i}$, take $\gamma^{1}(\beta)=\gamma$ and $\gamma^{2}(\beta)=\gamma^{\#}=\gamma$ (down); if $\gamma \notin \mathfrak{E}_{i}$, then take $\gamma^{1}(\beta)=\gamma^{2}(\beta)=p_{v}(\gamma)$.

Now suppose that $C$ has no element of $\mathfrak{O}$-depth $i$. Then, by Lemma 5.3.2 (4), $i$ is even and there exists in $C$ an element of $\mathfrak{O}$-depth $i-1$. This element of $C$ is of type $H$ by Lemma 5.3.2 (1), so $\mathfrak{E}$ is truly orthogonal at $i-1$. Set $\gamma^{1}(\beta)=\gamma^{2}(\beta)=p_{h}\left(\sigma_{i-1}\right)$.

(2): This proof parallels the proof of (1) above. As in the above proof, we may assume that $\beta=\beta$ (up). Suppose $\beta=(r, c)$ belongs to $\mathfrak{D} \mathfrak{E}_{j}^{\prime}$. Then there exists $(r, a) \in$ $\mathfrak{O E} \mathfrak{E}_{j}$ with $a \leq c$. Since $\mathfrak{O E}_{j}^{\prime}$ does not meet the diagonal, it is clear that $(r, a) \in$ $\mathfrak{O N}(v)$, and thus it is enough to prove the assertion for $\beta \in \mathfrak{O E}_{j}($ ext).

So now take $\beta \in \mathfrak{O E}_{j}(\mathrm{ext})$. Let $\beta^{\prime}$ and $C$ be in the proof of (1). First suppose that there exists in $C$ an element of $\mathfrak{O}$-depth $i$. Denote it by $\gamma$. If $\gamma \in \mathfrak{O} \mathfrak{E}_{i}$, then take

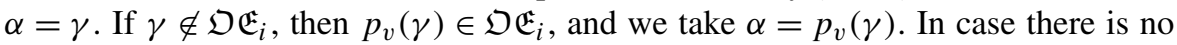
element in $C$ of $\mathfrak{O}$-depth $i$, we take $\alpha=p_{h}\left(\sigma_{i-1}\right)$ (see the above proof).

Corollary 8.3.6 If $\mathfrak{B}$ and $\mathfrak{B}_{1}$ are $\mathfrak{O}$-blocks of $\mathfrak{E}$ with $w(\mathfrak{B})=(r, c)$ and $w\left(\mathfrak{B}_{1}\right)=$ $\left(r_{1}, c_{1}\right)$, then exactly one of the following holds:

$$
\begin{array}{lll}
c<r<c_{1}<r_{1}, & & c_{1}<r_{1}<c<r, \\
c<c_{1}<r_{1}<r, & \text { or } & c_{1}<c<r<r_{1} .
\end{array}
$$

Proof This is a formal consequence of Lemmas 8.3.1 and 8.3.5.

Corollary 8.3.7 If $w(\mathfrak{B})>w(\mathfrak{C})$ for $\mathfrak{O}$-blocks $\mathfrak{B} \subseteq \mathfrak{O} \mathfrak{E}_{i}$ and $\mathfrak{C} \subseteq \mathfrak{O} \mathfrak{E}_{j}$ of $\mathfrak{E}$, then $i<j$.

Proof This is a formal consequence of Lemmas 8.3.1 and 8.3.5.

Corollary 8.3.8 Let $(s, t)>\left(s_{1}, t_{1}\right)$ be elements of $\mathfrak{O} \Pi_{\mathfrak{E}}$, and $\mathfrak{B}, \mathfrak{B}_{1}$ be $\mathfrak{O}$-blocks of $\mathfrak{E}$ such that $(s, t) \in \mathfrak{B}^{\prime}$, and $\left(s_{1}, t_{1}\right) \in \mathfrak{B}_{1}^{\prime}$. Then $w(\mathfrak{B})>w\left(\mathfrak{B}_{1}\right)$.

Proof Let $w(\mathfrak{B})=(r, c)$ and $w\left(\mathfrak{B}_{1}\right)=\left(r_{1}, c_{1}\right)$. By Corollary 8.3.6, we have four possibilities. Since $(r, c)$ dominates $(s, t)$ and $\left(r_{1}, c_{1}\right)$ dominates $\left(s_{1}, t_{1}\right)$, the possibilities $c<r<c_{1}<r_{1}$ and $c_{1}<r_{1}<c<r$ are eliminated. It is thus enough to 
eliminate the possibility $c_{1}<c<r<r_{1}$. Suppose that this is the case. Then, by Corollary 8.3.7, $j_{1}<j$, where $j_{1}$ and $j$ are such that $\mathfrak{B} \subseteq \mathfrak{O E}_{j}$ and $\mathfrak{B}_{1} \subseteq \mathfrak{O E}_{j_{1}}$. Now, by Lemma 8.3.5 (2), there exists $\alpha$ in $\mathfrak{O} \mathfrak{E}_{j_{1}}$ such that $\alpha>(s, t)>\left(s_{1}, t_{1}\right)$. But then this contradicts Lemma 8.3.2.

Corollary 8.3.9 For an $\mathfrak{O}$-block $\mathfrak{B} \subseteq \mathfrak{O E}_{i}$ of $\mathfrak{E}$, the depth of $w(\mathfrak{B})$ in $\mathfrak{S}_{\mathfrak{O}} w_{\mathfrak{E}}$ is exactly $i$.

Proof Lemma 8.3.5 and Corollary 8.3.7.

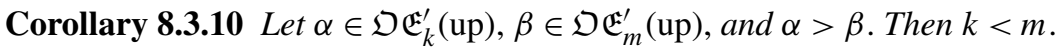

Proof Corollary 8.3.8 and Corollary 8.3.9.

\subsection{More lemmas}

This subsection is a collection of lemmas to be invoked in the later subsections. More specifically, Lemma 8.4.1 and Corollary 8.4.2 below are invoked in the proof of Proposition 4.3.1 in \$9.1, Lemma 8.4.3 in the proof of the first half of Proposition 4.3.2 in $\$ 9.2$, and Lemma 8.4.4 in the proof of the second half of Proposition 4.3.2 in $\$ 9.3$. The lemmas may make sense only when one tries to read the proofs of the propositions.

Throughout this subsection, $\mathfrak{E}$ denotes a monomial in $\mathfrak{O N}(v)$.

Lemma 8.4.1 Let $C$ be a $v$-chain in $\mathfrak{O} \Pi_{\mathfrak{E}}, \alpha$ an element of $C$, and $\alpha^{\prime} \in \mathfrak{O m o n}_{C, \alpha}$. Then $\operatorname{depth}_{\mathfrak{O} \mathfrak{m o n}_{C}} \alpha^{\prime} \leq k$ (even), for $k$ the integer such that $\alpha \in \mathfrak{O E}_{k}^{\prime}$ (up).

Proof Proceed by induction on $k$. If $k=1$, the assertion follows from Corollary 8.3.10, so assume $k>1$. Choose a $v$-chain $C^{\prime}$ in $\mathfrak{O m o n}_{C}$ with tail $\alpha^{\prime}$ and $\operatorname{depth}_{C^{\prime}} \alpha^{\prime}=\operatorname{depth}_{\mathfrak{O m o n}} \alpha^{\prime}$. The length of a $v$-chain in $\mathfrak{O m o n}_{C, \alpha}$ is clearly at most 2 . So, if $\gamma^{\prime}$ is the element two steps before $\alpha^{\prime}$ in $C^{\prime}$ (if $\gamma^{\prime}$ does not exist then there is clearly nothing to prove), then $\gamma^{\prime} \in \mathfrak{O m o n}_{C, \gamma}$ with $\gamma>\alpha$ (see Proposition 5.1.4 (2)). We claim that depth $\mathfrak{O m o n}_{C} \gamma^{\prime} \leq k$ (odd) -1 . It is enough to prove the claim, for then $\operatorname{depth}_{\mathfrak{O m o n} \mathfrak{m}_{C}} \alpha^{\prime}=\operatorname{depth}_{C^{\prime}} \alpha^{\prime}=\operatorname{depth}_{C^{\prime}} \gamma^{\prime}+2 \leq k$ (odd) $-1+2=k($ even $)$.

The claim follows by induction from Corollary 8.3.10 if $k$ is odd or more generally if $\gamma \in \mathfrak{O} \mathfrak{E}_{l}^{\prime}$ (up) with $l \leq k$ (odd) -1 . So assume that $k$ is even and $\gamma \in \mathfrak{O} \mathfrak{E}_{k-1}^{\prime}$ (up). By 6.5.4, it is not possible that $\gamma$ is of type $\mathrm{H}$ and $p_{h}(\gamma)>\alpha$. So the only possibility is that $\alpha^{\prime}=p_{h}(\alpha)$ and $\gamma>\alpha$ is connected. In particular, $\gamma$ is of type $\mathrm{V}$ and $\alpha$ of type $\mathrm{H}$ in $C$ and $\gamma^{\prime}=p_{v}(\gamma)$.

Now let $\mu$ be the first element in the connected component of $\alpha$ in $C$. The cardinality of the part $\mu>\ldots>\gamma$ of $C$ is even (by Proposition 5.1.1 (1), it follows that

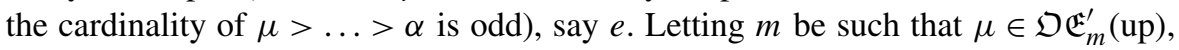
we have, by Proposition 8.3.10, $m \leq k-1-(e-1)=k-e$. If $m$ (even) $<k-e$, then, since $\operatorname{depth}_{C^{\prime}} \gamma^{\prime}=\operatorname{depth}_{C^{\prime}} p_{v}(\mu)+e-1$ (by Proposition 5.1 .4 (1), since, by Proposition 5.1.1 (2), $\mu, \ldots, \gamma$ all have type $\mathrm{V}$ in $C$ ) and $\operatorname{depth}_{C^{\prime}} p_{v}(\mu) \leq m$ (even) by induction, it follows that depth $C_{C^{\prime}} \gamma^{\prime}<k-e+e-1=k-1$, and we are done. 
So suppose that $m$ (even) $=k-e$. Let $v$ be the element just before $\mu$ in $C$ (if such an element does not exist, then $\operatorname{depth}_{C^{\prime}} \gamma^{\prime}=e \leq k-2$-observe that $m$ (even) $\geq 2$ and we are done). Then $v>\mu$ is not connected (by choice of $\mu$ ). So $p_{h}(v)>\mu$. By Proposition 6.5.4, this means that $j \leq m$ (even) -2 where $j$ is the odd integer defined by $v \in \mathfrak{O E} \mathfrak{E}_{j}^{\prime}$ (up) $\cup \mathfrak{O E _ { j + 1 } ^ { \prime }}$ (up). So, again by induction, $\operatorname{depth}_{C^{\prime}} \gamma^{\prime}=\operatorname{depth}_{C^{\prime}} p_{h}(v)+$ $e \leq m$ (even) $-2+e=k-2$, and the claim is proved.

Corollary 8.4.2 The $\mathfrak{O}$-depth of an element $\alpha$ in $\mathfrak{O} \Pi_{\mathfrak{E}}$ is at most $k$ where $k$ is such that $\alpha \in \mathfrak{O} \mathfrak{E}_{k}^{\prime}$ (up).

Proof Let $C^{\prime}$ be a $v$-chain in $\mathfrak{O m o n}{ }_{C}$ with tail $\mathfrak{O} q_{C, \alpha}$. If $k$ is even, then, by the lemma, depth $C_{C^{\prime}} \mathfrak{O} q_{C, \alpha} \leq k$. So suppose that $k$ is odd. Let $\gamma^{\prime}$ be the immediate predecessor of $\mathfrak{O} q_{C, \alpha}$ in $C^{\prime}$. By Proposition 5.1.4 (2), $\gamma>\alpha$, and so $\gamma \in \mathfrak{O} \mathfrak{E}_{l}^{\prime}$ (up) with $l \leq k-1$ (see the observation in the first paragraph of the proof of the lemma). So $\operatorname{depth}_{C^{\prime}} \gamma^{\prime} \leq k-1$ (by the lemma) and depth ${ }_{C^{\prime}} \alpha^{\prime}=\operatorname{depth}_{C^{\prime}} \gamma^{\prime}+1 \leq k$.

Lemma 8.4.3 Let $\mathfrak{E}$ be a monomial in $\mathfrak{O N}(v)$ and $\mathfrak{O} \pi(\mathfrak{E})=\left(\mathfrak{O} w_{\mathfrak{E}}, \mathfrak{O} \Pi_{\mathfrak{E}}\right)$. Let $i<k$ be integers, $\alpha$ an element of $\mathfrak{O} \mathfrak{E}_{i}^{\prime}(\mathrm{up})$, and $\delta$ an element of $\left(\mathfrak{S}_{\mathfrak{O} w_{\mathfrak{E}}}\right)_{k}(\mathrm{up})$ that dominates $\alpha$.

(1) If $k$ is even, then there exists $\beta \in \mathfrak{O} \mathfrak{E}_{k}^{\prime}(\mathrm{up})$ with $\alpha>\beta$.

(2) If $k$ is odd and $\left(\mathfrak{O} w_{\mathfrak{E}}\right)_{k, k+1} \mathfrak{O}$-dominates the singleton $v$-chain $\alpha$, then either there exists $\beta \in \mathfrak{O} \mathfrak{E}_{k}^{\prime}(\mathrm{up})$ with $\alpha>\beta$ or there exists $\gamma \in \mathfrak{O} \mathfrak{E}_{k+1}^{\prime}$ (up) with $p_{h}(\alpha)>\gamma$.

Proof Write $\alpha=(r, c)$ and $\delta=(A, B)$. By Corollary 8.3.9, there exists a block $\mathfrak{B}$ of $\mathfrak{O E}_{k}$ such that $\delta=w(\mathfrak{B})$. Let $(D, B)$ be the first element of $\mathfrak{B}$ (arranged in increasing order of row and column indices). We have the following possibilities:

(i) $D \leq A$ and $(D, B) \in \mathfrak{O} \mathfrak{E}_{k}^{\mathrm{pr}}$.

(ii) $k$ is odd, $\mathfrak{E}$ is truly orthogonal at $k,(D, B)=(A, B)=p_{v}\left(\sigma_{k}\right)$, and $\mathfrak{B}$ consists of the single diagonal element $(D, B)=\left(B^{*}, B\right)$.

(iii) $k$ is even, $\mathfrak{E}$ is truly orthogonal at $k-1,(D, B)=(A, B)=p_{h}\left(\sigma_{k-1}\right)$, and $\mathfrak{B}$ consists of the single diagonal element $(D, B)=\left(B^{*}, B\right)$.

We claim the following: in case (i), $D<r$ (in particular, $D<A$ ); in case (ii), the row index of $\sigma_{k}$ is less than $r$; and case (iii) is not possible. The first two assertions and also the third in the case $i<k-1$ follow readily from Lemma 8.3.3; in case (iii) holds and $i=k-1$, then $\sigma_{k-1}>\alpha$, a contradiction to Lemma 8.3.2.

First suppose that possibility (ii) holds. Write $\sigma_{k}=(s, B)$. Since $s<r$ and $p_{h}\left(\sigma_{k}\right) \in \mathfrak{N}(v)$, it is clear that $p_{h}(\alpha)=\left(r, r^{*}\right)$ also belongs to $\mathfrak{N}(v)$. From the hypothesis that $\left(\mathfrak{O} w_{\mathfrak{E}}\right)_{k, k+1} \mathfrak{O}$-dominates $\{\alpha\}$, it follows that there is an element of $\left(\mathfrak{S}_{\mathfrak{O} w_{\mathfrak{E}}}\right)_{k+1}$ that dominates $p_{h}(\alpha)=\left(r, r^{*}\right)$. Such an element must be diagonal (because of the distinguishedness of $\left.\mathfrak{S}_{\mathfrak{O} w_{\mathfrak{E}}}\right)$, and so must be the $w(\mathfrak{C})$ for the unique diagonal block $\mathfrak{C}$ of $\mathfrak{O} \mathfrak{E}_{k+1}$. In particular, this means that there are elements other than $\left(s, s^{*}\right)$ in $\mathfrak{O} \mathfrak{E}_{k+1}$, and so $\mathfrak{O} \mathfrak{E}_{k+1}^{\prime}$ is non-empty. In the arrangement of elements of $\mathfrak{O} \mathfrak{E}_{k+1}^{\prime}$ (up) in increasing order of row and column numbers, let $\gamma=\left(e, s^{*}\right)$ be the last element. Then $e<s<r$ and $r^{*}<s^{*}$, so $p_{h}(\alpha)>\gamma$, and we are done. 
Now suppose that possibility (i) holds. Let $(p, q)$ be the element of $\mathfrak{O E}_{k}$ such that $p$ is the largest row index that is less than $r$, and, among those elements with row index $p$, the maximum possible column index is $q$. The arrangement of elements of $\mathfrak{O E} \mathfrak{E}_{k}$ (in increasing order or row and column indices) looks like this:

$$
\ldots,(p, q),(s, t), \ldots
$$

Since $p<r \leq A$ and $w(\mathfrak{B})=(A, B)$, we can be sure that $(p, q)$ is not the last element of $\mathfrak{B}$.

We first consider the case $c<t$. Then $\alpha=(r, c)>\beta:=(p, t) \in \mathfrak{O} \mathfrak{E}_{k}^{\prime}$. If $\beta \in$ $\mathfrak{O \mathfrak { E } _ { k } ^ { \prime }}$ (up), then we are done. It is possible that $(p, q)$ lies on or below the diagonal so that $\beta$ lies below the diagonal, in which case, $\alpha>\beta$ (up) and $\beta$ (up) $\in \mathfrak{O} \mathfrak{E}_{k}^{\prime}$ (up), and again we are done.

Now suppose that $t \leq c$. We claim that:

- $(s, t)$ belongs to the diagonal;

- $k$ is odd and $\mathfrak{E}$ is truly orthogonal at $k$; and

- $\sigma_{k}=(u, t)$ with $u<r$.

Suppose that $(s, t)$ does not belong to the diagonal. Since $r \leq s$ (by choice of $(p, q)$ ), it follows that $(s, t)$ dominates $(r, c)$. This leads to a contradiction to Lemma 8.3.3, for either $(s, t)$ or its reflection $\left(t^{*}, s^{*}\right)$ (whichever is above the diagonal) belongs

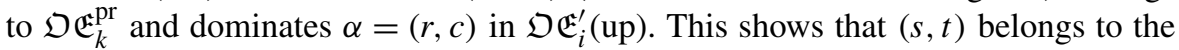
diagonal. If $k$ is even, then $(s, t)=p_{h}\left(\sigma_{k-1}\right)$, which means $\sigma_{k-1}>\alpha$, again contradicting Lemma 8.3.3, so $k$ must be odd. It also follows that $\mathfrak{E}$ is truly orthogonal at $k$ and that $(s, t)=p_{v}\left(\sigma_{k}\right)$. Writing $\sigma_{k}=(u, t)$, if $r \leq u$, then $\sigma_{k}$ would dominate $\alpha$, again contradicting Lemma 8.3.3. So $u<r$, and the claim is proved.

To finish the proof of the lemma, now proceed as in the proof when possibility (ii) holds.

Lemma 8.4.4 Let $\mathfrak{T}$ be a monomial in $\mathfrak{O N}(v)$ and $w$ an element of $\mathfrak{O} I(d)$ that $\mathfrak{O}$-dominates $\mathfrak{T}$. Let $\beta^{\prime}>\beta$ be elements $\mathfrak{S}_{w}(\mathrm{up})$. Let $d-1$ and $d$ be their respective depths in $\mathfrak{S}_{w}$. Let $\alpha$ be an element of $\mathfrak{O}(\mathfrak{T}, w, \beta)^{\star}$ or more generally an element of $\mathfrak{O N}(v)$ such that

(a) it is dominated by $\beta$,

(b) it is not comparable to any element of $\mathfrak{D P}_{\beta}$, and

(c) in case $d$ is odd, then $\{\alpha\} \cup \mathfrak{O T} \mathfrak{T}_{w, d, d+1}$ has $\mathfrak{O}$-depth at most 2.

Then

(1) there exists $\alpha^{\prime} \in \mathfrak{O P}_{\beta^{\prime}}^{*}$ (up) with $\alpha^{\prime}>\alpha$;

(2) for $\alpha^{\prime}$ as in (1), if $\alpha^{\prime}$ is diagonal, then $p_{h}\left(\delta_{d-2}\right)>\alpha$ if $d$ is odd and $\delta_{d-1}>\alpha$ if $d$ is even.

Proof Assertion (2) is rather easy to prove. If $d$ is odd, then, in fact, $p_{h}\left(\delta_{d-2}\right)=\alpha^{\prime}$; if $d$ is even, then $\delta_{d-1}$ has the same column index as $\alpha^{\prime}$ and, by Proposition 7.2.2 (4), has row index more than that of $\alpha$, so $\delta_{d-1}>\alpha$.

Let us prove (1). Write $\alpha=(r, c), \beta=(R, C)$, and $\beta^{\prime}=\left(R^{\prime}, C^{\prime}\right)$. There exists, by the definition of $\mathfrak{O P}_{\beta^{\prime}}^{*}$, an element in $\mathfrak{O P}_{\beta^{\prime}}^{*}$ with column index $C^{\prime}$. We have $C^{\prime}<c$ 
(for $C^{\prime}<C \leq c$ ). Let $\left(r^{\prime}, c^{\prime}\right)$ be the element of $\mathfrak{O P}_{\beta^{\prime}}^{*}$ such that $c^{\prime}$ is maximum possible subject to $c^{\prime}<c$ and among those elements with column index $c^{\prime}$ the maximum possible row index is $r^{\prime}$. If $r<r^{\prime}$, then we are done (if $\left(r^{\prime}, c^{\prime}\right)$ is below the diagonal, its mirror image would have the desired properties). It suffices therefore to suppose that $r^{\prime} \leq r$ and arrive at a contradiction.

In the arrangement of elements of $\mathfrak{O P}_{\beta^{\prime}}^{*}$ in non-decreasing order of row and column indices, there is a portion that looks like this:

$$
\ldots,\left(r^{\prime}, c^{\prime}\right),(a, b), \ldots
$$

Since there is in $\mathfrak{O}(\mathfrak{T}, w, \beta)^{\star}$ an element with row index $R^{\prime}$ (and clearly $r^{\prime} \leq r<R<$ $\left.R^{\prime}\right)$, it follows that $(a, b)$ exists (that is, $\left(r^{\prime}, c^{\prime}\right)$ is not the last element in the above arrangement). It follows from the construction of $\mathfrak{O P}_{\beta^{\prime}}^{*}$ from $\mathfrak{O P}_{\beta^{\prime}}$ that $\left(r^{\prime}, b\right)$ is an element in $\mathfrak{O P} \beta_{\beta^{\prime}}$. By the choice of $\left(r^{\prime}, c^{\prime}\right)$, we have $c \leq b$. Thus $(r, c)$ dominates $\left(r^{\prime}, b\right)$.

The proof now splits into two cases accordingly as $d$ is even or odd. First suppose that $d$ is even. Then, since $\beta$ dominates $\left(r^{\prime}, b\right)$ and yet $\left(r^{\prime}, b\right)$ does not belong to $\mathfrak{O P}_{\beta}$, there exists a $v$-chain in $\mathfrak{T}_{w, d-1, d}$ of length 2 and head $\left(r^{\prime}, b\right)$. The tail of this $v$-chain then belongs to $\mathfrak{O P}_{\beta}$ and is dominated by $(r, c)$, a contradiction to our assumption that $\alpha$ is not comparable to any element of $\mathfrak{O P}_{\beta}$.

Now suppose that $d$ is odd. Choose a $v$-chain $C$ in $\mathfrak{T}$ with head $\left(r^{\prime}, b\right)$ that is not $\mathfrak{O}$-dominated by $w^{d}$. Let $D$ be the part of $C$ consisting of elements of $\mathfrak{O}$-depth (in $C$ ) at most 2 . We claim that $D$ is $\mathfrak{O}$-dominated by $w_{d, d+1}$. In fact, we claim the following: Any $v$-chain $F$ with head $\left(r^{\prime}, b\right)$ and $\mathfrak{O}$-depth at most 2 is $\mathfrak{O}$-dominated by $w_{d, d+1}$.

To prove the claim, we first prove the following sub-claim:

$(\dagger)$ If the horizontal projection of $\left(r^{\prime}, b\right)$ belongs to $\mathfrak{N}(v)$, then $\beta$ is on the diagonal and dominates the vertical projection of $\left(r^{\prime}, b\right)$, and the diagonal element $\beta_{1}$ of $\left(\mathfrak{S}_{w}\right)_{d+1}$ dominates the horizontal projection of $\left(r^{\prime}, b\right)$.

Let $p_{h}\left(r^{\prime}, b\right) \in \mathfrak{N}(v)$. Then $\beta$ belongs to the diagonal because $\mathfrak{S}_{w}$ is distinguished and symmetric. Once $\beta$ is on the diagonal, it is clear that it dominates $p_{v}\left(r^{\prime}, b\right)$ (from our assumptions, $\beta$ dominates $(r, c)$ and $(r, c)$ dominates $\left.\left(r^{\prime}, b\right)\right)$. It follows from Proposition 7.2.2 (3) that the row index of $\beta_{1}$ exceeds the row index $r$ of $(r, c)$, so $\beta_{1}$ dominates $p_{h}\left(r^{\prime}, b\right)$. This finishes the proof of the sub-claim $(\dagger)$.

To begin the proof of the claim, observe that $F$ has length at most 2. Suppose first that $F$ consists only of the single element $\left(r^{\prime}, b\right)$. The type of $\left(r^{\prime}, b\right)$ in $F$ is either $\mathrm{H}$ or $\mathrm{S}$. If it is $\mathrm{S}$, then since $\beta$ dominates $\left(r^{\prime}, b\right)$, the claim follows immediately. If it is $\mathrm{H}$, then the claim follows immediately from the sub-claim $(\dagger)$.

Continuing with the proof of the claim, let now $F$ consist of two elements: $\left(r^{\prime}, b\right)>\mu$. Let $\gamma$ be the element of $\mathfrak{S}_{w}$ such that $\mu \in \mathfrak{O P}_{\gamma}$, and let $e$ be the depth of $\gamma$ in $\mathfrak{S}_{w}$. From Lemma 7.2.1 (2b) it follows that $e \geq d$. If $e=d$, then $\gamma=\beta$ (by the distinguishedness of $\left.\mathfrak{S}_{w}\right)$, and the comparability of $(r, c)$ and $\mu$ contradicts our hypothesis (b). So $e \geq d+1$, and there exists $\delta$ of depth $d+1$ in $\mathfrak{S}_{w}$ that dominates $\mu$. We have $\beta>\delta$ (again by the distinguishedness of $\mathfrak{S}_{w}$ ).

The possibilities for the types of $\left(r^{\prime}, b\right)$ and $\mu$ in $F$ are: $\mathrm{S}$ and $\mathrm{S}, \mathrm{V}$ and $\mathrm{V}, \mathrm{H}$ and $\mathrm{S}$ (in the last case $p_{h}\left(r^{\prime}, b\right) \ngtr \mu$ by Lemma 5.3.2 (1)). Noting the existence in 
$\left(\mathfrak{S}_{w}\right)_{d, d+1}$ of the $v$-chain $\beta>\delta$ in the first case and also of $\beta>\beta_{1}$ (where $\beta_{1}$ is as in the sub-claim) in the last case, the proof of the claim in these cases is over. So suppose that the second possibility holds. The distinguishedness of $\mathfrak{S}_{w}$ implies that $\delta=\beta_{1}$. Since $\delta$ is diagonal, it dominates the vertical projection of $\mu$. Noting the existence of the $v$-chain in $\beta>\delta$ in $\left(\mathfrak{S}_{w}\right)_{d, d+1}$, the proof of the claim in this case too is over.

We continue with the proof of the lemma. It follows from the claim that $D$ is $\mathfrak{O}$-dominated by $w_{d, d+1}$. From Corollary 8.2.3 it follows that the complement $E$ of $D$ in $C$ is not $\mathfrak{O}$-dominated by $w_{d+2, d+3}$ (in particular, that $E$ is non-empty) and that every $v$-chain in $\mathfrak{T}$ with head $\epsilon$ (where $\epsilon$ denotes the head of $E$ ) is $\mathfrak{O}$-dominated by $w^{d}$ (given such a $v$-chain, the concatenation of $D$ with it is $\mathfrak{O}$-dominated by $w^{d-2}$, and $\epsilon$ continues to have $\mathfrak{D}$-depth 3 in the concatenated $v$-chain). Thus $\epsilon$ belongs to $\mathfrak{D T} \mathfrak{T}_{w, d, d+1}$. From (1) and (2b) of Lemma 7.2.1 it follows that the element $\mu$ of $C$ in between $\left(r^{\prime}, b\right)$ and $\epsilon$ (if it exists at all) also belongs to $\mathfrak{D T} \mathfrak{T}_{w, d, d+1}$. Now consider the $v$-chain obtained as follows: take the part of $C$ up to (and including) $\epsilon$ and replace its head $\left(r^{\prime}, b\right)$ by $(r, c)$. This chain has $\mathfrak{O}$-depth 3 and lives in $\{\alpha\} \cup \mathfrak{O T} \mathfrak{T}_{w, d, d+1}$, a contradiction to hypothesis (c).

Corollary 8.4.5 Let $\mathfrak{T}$ be a monomial in $\mathfrak{O N}(v)$ and $w$ an element of $\mathfrak{O} I(d)$ that $\mathfrak{O}$-dominates $\mathfrak{T}$. Let $\beta^{\prime}>\beta$ be elements of $\mathfrak{S}_{w}(\mathrm{up}), \alpha$ an element of $\mathfrak{D}(\mathfrak{T}, w, \beta)^{\star}$, and $d^{\prime}:=\operatorname{depth}_{\mathfrak{S}_{w}} \beta^{\prime}$.

(1) If $d^{\prime}$ is odd, there exists $\alpha^{\prime} \in \mathfrak{O P}_{\beta^{\prime}}^{*}$ such that $\alpha^{\prime}>\alpha$.

(2) If there does not exist $\alpha^{\prime} \in \mathfrak{O P}_{\beta^{\prime}}^{*}$ such that $\alpha^{\prime}>\alpha$ then $\left(d^{\prime}\right.$ is even by (1) above and) there exists $\alpha^{\prime \prime} \in \mathfrak{O P}_{\beta^{\prime \prime}}^{*}$ such that $p_{h}\left(\alpha^{\prime \prime}\right)>\alpha$, where $\beta^{\prime \prime}$ is the unique element of $\left(\mathfrak{S}_{w}\right)_{d^{\prime}-1}$ such that $\beta^{\prime \prime}>\beta^{\prime}$.

Corollary 8.4.6 Let $\mathfrak{T}$ be a monomial in $\mathfrak{O N}(v)$ and $w$ an element of $\mathfrak{O} I(d)$ that $\mathfrak{O}$-dominates $\mathfrak{T}$. Let $\beta, \beta^{\prime}$ be elements of $\mathfrak{S}_{w}(\mathrm{up})$, and $\alpha, \alpha^{\prime}$ elements of $\mathfrak{O}(\mathfrak{T}, w, \beta)^{\star}$ and $\mathfrak{O P}_{\beta^{\prime}}^{*}$ respectively.

(1) If $\alpha^{\prime}>\alpha$ then $\beta^{\prime}>\beta$ (in particular, $\operatorname{depth}_{\mathfrak{S}_{w}} \beta^{\prime}<\operatorname{depth}_{\mathfrak{S}_{w}} \beta$ ).

(2) If $p_{h}\left(\alpha^{\prime}\right)>\alpha$ and $\operatorname{depth}_{\mathfrak{S}_{w}} \beta$ is even, $\operatorname{depth}_{\mathfrak{S}_{w}} \beta^{\prime} \leq \operatorname{depth}_{\mathfrak{S}_{w}} \beta-2$.

Proof (1) Writing $\beta=(r, c)$ and $\beta^{\prime}=\left(r^{\prime}, c^{\prime}\right)$, there are, since both $\beta$ and $\beta^{\prime}$ dominate $\alpha$ and $\mathfrak{S}_{w}$ is distinguished, the following four possibilities:

$$
c<r<c_{1}<r_{1}, \quad c_{1}<r_{1}<c<r, \quad c<c_{1}<r_{1}<r, \quad c_{1}<c<r<r_{1}
$$

Since $\alpha^{\prime}>\alpha$, and $\alpha, \alpha^{\prime}$ are dominated respectively by $\beta, \beta^{\prime}$ (this is because $\alpha, \alpha^{\prime}$ belong to $\mathfrak{O}(\mathfrak{T}, w, \beta)^{\star}, \mathfrak{O P}_{\beta^{\prime}}^{*}$ respectively), the possibilities $c<r<c_{1}<r_{1}$ and $c_{1}<r_{1}<c<r$ are eliminated (by the distinguishedness of $\mathfrak{S}_{w}$ ). It is thus enough to eliminate the possibility $\beta>\beta^{\prime}$. Suppose, by way of contradiction, that $\beta>\beta^{\prime}$. By Corollary 8.4.5, either there exists $\gamma \in \mathfrak{O}(\mathfrak{T}, w, \beta)^{\star}$ such that $\gamma>\alpha^{\prime}$, in which case the $v$-chain $\gamma>\alpha$ in $\mathfrak{O}(\mathfrak{T}, w, \beta)^{\star}$ contradicts Proposition 7.2.2 (3) or (4), or $d:=\operatorname{depth}_{\mathfrak{S}_{w}} \beta$ is even and there exists (with $\beta^{\prime \prime}$ being the unique element in $\mathfrak{S}_{w}$ such that $\beta^{\prime \prime}>\beta$ and $\left.\operatorname{depth}_{\mathfrak{S}_{w}} \beta^{\prime \prime}=d-1\right)$ an element $\alpha^{\prime \prime} \in \mathfrak{O P}_{\beta^{\prime \prime}}^{*}$ with $p_{h}\left(\alpha^{\prime \prime}\right)>\alpha^{\prime}$, 
in which case the $v$-chain $\alpha^{\prime \prime}>\alpha$ in $\mathfrak{O} \mathfrak{T}_{w, d-1, d}^{\star}$ has $\mathfrak{O}$-depth 3 and so contradicts Proposition 7.2.2 (2).

(2) Set $d:=\operatorname{depth}_{\mathfrak{S}_{w}} \beta$. If $\operatorname{depth}_{\mathfrak{S}_{w}} \beta^{\prime}$ were $d-1$, then the $v$-chain $\alpha^{\prime}>\alpha$ in $\mathfrak{D T} \mathfrak{T}_{w, d-1, d}^{\star}$ would be of $\mathfrak{O}$-depth 3 and so would contradict Proposition 7.2.2 (2).

\section{The Proof}

We are at last ready to prove the propositions of $\S 4.3$. The proof of the first proposition appears in $\S 9.1$ and that of the second in $\S \S 9.2,9.3$. These rely on the lemmas of $\S 8.4$. As pointed out in the beginning of $\S 8.4$, the lemmas may make no sense until one tries to read the proofs here.

\subsection{Proof of Proposition 4.3.1}

(1) By definition, $\mathfrak{O} w_{\mathfrak{E}}$ is the element of $\mathfrak{O} I(d)$ associated to the distinguished

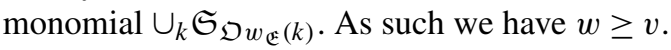

(2) follows from the corresponding property of the map $\pi$ of [7]. More precisely, that property justifies the third equality below; the other equalities are clear from the definitions:

$$
\begin{aligned}
& \mathfrak{O}-v \text {-degree }\left(\mathfrak{O} w_{\mathfrak{E}}\right)+\operatorname{degree}\left(\mathfrak{O} \Pi_{\mathfrak{E}}\right)=\frac{1}{2} \operatorname{degree}\left(\mathfrak{S}_{\mathfrak{O} w_{\mathfrak{E}}}\right)+\frac{1}{2} \sum_{k} \operatorname{degree}\left(\mathfrak{O} \mathfrak{E}_{k}^{\prime}\right) \\
& =\frac{1}{2} \sum_{k}\left(\operatorname{degree}\left(\mathfrak{S}_{\mathfrak{O} w_{\mathfrak{E}}(k)}\right)+\operatorname{degree}\left(\mathfrak{O} \mathfrak{E}_{k}^{\prime}\right)\right)=\frac{1}{2} \sum_{k} \operatorname{degree}\left(\mathfrak{O} \mathfrak{E}_{k}\right)
\end{aligned}
$$

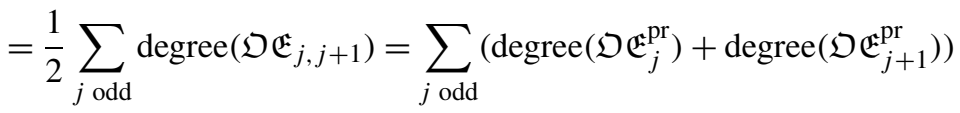

$$
\begin{aligned}
& =\text { degree(E). }
\end{aligned}
$$

(3) The first equivalence below follows from the definition of $\mathfrak{O}$-domination, the second from [7, Lemma 4.5], the third from Lemma 8.1.3:

$$
\begin{aligned}
\mathfrak{O} w_{\mathfrak{E}} \mathfrak{O} \text {-dominates } \mathfrak{O} \Pi_{\mathfrak{E}} & \Leftrightarrow \quad \mathfrak{O} w_{\mathfrak{E}} \geq \mathfrak{O} w_{C} \forall v \text {-chain } C \text { in } \mathfrak{O} \Pi_{\mathfrak{E}} \\
& \Leftrightarrow \quad \mathfrak{O} w_{\mathfrak{E}} \text { dominates } \mathfrak{O m o n} C \forall v \text {-chain } C \text { in } \mathfrak{O} \Pi_{\mathfrak{E}} \\
& \Leftrightarrow \quad \forall v \text {-chain } C \text { in } \mathfrak{O} \Pi_{\mathfrak{E}}, \forall \alpha^{\prime}=(r, c) \in \mathfrak{O m o n} C, \\
& \exists \beta=(R, C) \in \mathfrak{S}_{\mathfrak{O} w_{\mathfrak{E}}} \text { with } C \leq c, r \leq R, \\
& \quad \text { and depth }_{\mathfrak{S}_{\mathfrak{O} w_{\mathfrak{E}}}} \beta \geq \operatorname{depth}_{\mathfrak{O} \mathfrak{m o n}_{C}} \alpha^{\prime} .
\end{aligned}
$$

Now let $C$ be a $v$-chain in $\mathfrak{O} \Pi_{\mathfrak{E}}$ and $\alpha^{\prime}=(r, c)$ in $\mathfrak{O m o n}_{C}$. We will show that there exists $\beta$ in $\mathfrak{S}_{w}$ that dominates $\alpha$ and satisfies $\operatorname{depth}_{\mathfrak{S}_{w}} \beta \geq \operatorname{depth}_{\mathfrak{O m o n} \mathfrak{m}_{C}} \alpha^{\prime}$. Let $\alpha$ be the element in $C$ such that $\alpha^{\prime} \in \mathfrak{O m o n}_{C, \alpha}$, let $k$ be such that $\alpha \in \mathfrak{O} \mathfrak{E}_{k}^{\prime}$ (up), and let $\mathfrak{B}$ be the block of $\mathfrak{O \mathfrak { E } _ { k }}$ such that $\alpha \in \mathfrak{B}^{\prime}$. Writing $\alpha=\left(r_{1}, c_{1}\right)$ and $w(\mathfrak{B})=\left(R_{1}, C_{1}\right)$, we have $C_{1} \leq c_{1}$ and $r_{1} \leq R_{1}$ straight from the definition of $w(\mathfrak{B})$. By Corollary 8.3.9, $\operatorname{depth}_{\mathfrak{S}_{\mathfrak{O} w_{\mathfrak{E}}}} w(\mathfrak{B})=k$. 
First suppose that $w(\mathfrak{B})$ dominates $\alpha^{\prime}$ (meaning $C_{1} \leq c$ and $r \leq R_{1}$ ). If $k \geq$ $\operatorname{depth}_{\mathfrak{O} \mathfrak{m o n}_{C}} \alpha^{\prime}$, we are clearly done; by Corollary 8.4.2, this is the case when $\alpha^{\prime}=\mathfrak{O} q_{C, \alpha}$. So suppose that $\alpha$ is of type $\mathrm{H}, \alpha^{\prime}=p_{h}(\alpha)$, and that $k<\operatorname{depth}_{\mathfrak{O m o n} C} \alpha^{\prime}$. By Lemma 8.4.1, depth $\mathfrak{O m o n}_{C} \alpha^{\prime} \leq k$ (even). It follows that $k$ is odd and that $\operatorname{depth}_{\mathfrak{O m o n}_{C}} \alpha^{\prime}=k+1$. By Corollary 6.5.3, E is truly orthogonal at $k$, which means that $\mathfrak{O} \mathfrak{E}_{k+1}$ has a diagonal block, say $\mathfrak{C}$. Note that $w(\mathfrak{C})$ dominates $p_{h}\left(\sigma_{k}\right)$ which in turn dominates $p_{h}(\alpha)$. Since depth $\mathfrak{S}_{\mathfrak{O} w_{\mathfrak{E}}} w(\mathfrak{C})=k+1$ by Corollary 8.3 .9 , we are done.

Now suppose that $w(\mathfrak{B})$ does not dominate $\alpha^{\prime}$. Then $\mathfrak{B}$ is non-diagonal and $\alpha^{\prime}=$ $p_{v}(\alpha)$. Since $\mathfrak{B}$ is non-diagonal, $p_{h}(\alpha) \notin \mathfrak{N}(v)$, and $\alpha$ cannot be of type H. So $\alpha$ is of type $\mathrm{V}$ in $C$. It follows easily (see Proposition 5.1.1 (3)) that $\alpha$ is the critical element in $C$ and the last in its connected component in $C$; by Lemma 5.3.2 (3), $\mathfrak{O}$-depth ${ }_{C}(\alpha)=$ depth $_{\mathfrak{O m o n}} \mathfrak{O} q_{C, \alpha}=: d$ is even. By Proposition 5.1.1 (1), (2), the cardinality of the connected component of $\alpha$ in $C$ is even. In particular, the immediate predecessor in $C$ of $\alpha$-call it $\gamma$-is connected to $\alpha$.

The type of $\gamma$ in $C$ is $\mathrm{V}, p_{h}(\gamma)$ belongs to $\mathfrak{N}(v)$, and $\operatorname{depth}_{\mathfrak{O m o n}} p_{v}(\gamma)=d-1$ (see Lemma 5.3.2 (1)). Let $\ell$ be such that $\gamma \in \mathfrak{O} \mathfrak{E}_{\ell}^{\prime}$ (up). Let $\mathfrak{C}$ be the block of $\mathfrak{O} \mathfrak{E}_{\ell}$ such that $\gamma \in \mathfrak{C}^{\prime}$. Since $p_{h}(\gamma) \in \mathfrak{N}(v), \mathfrak{C}$ is diagonal. Note that $w(\mathfrak{C})$ dominates $p_{v}(\gamma)$ and that $p_{v}(\gamma)>p_{v}(\alpha)$. By Corollary 8.3.9, $\operatorname{depth}_{\mathfrak{S}_{\mathfrak{O} w_{\mathfrak{E}}}} w(\mathfrak{C})=\ell$. Thus if $d \leq \ell$ we are done. On the other hand, $d-1 \leq \ell$ by Corollary 8.4.2.

So we may assume that $\ell=d-1$. By Corollary 6.5 .3 , $\mathfrak{E}$ is truly orthogonal at $d-1$. This implies that $\mathfrak{O E}_{d}$ has a diagonal block, say $\mathfrak{D}$. Note that $w(\mathfrak{D})$ dominates $p_{h}\left(\sigma_{d-1}\right)$ which in turn dominates $p_{h}(\gamma)$. Writing $\gamma=\left(r_{2}, c_{2}\right)$, since $\gamma>\alpha$ is connected, it follows that $\left(r_{1}, r_{2}^{*}\right)$ belongs to $\mathfrak{O N}(v)$. Now both $w(\mathfrak{B})$ and $w(\mathfrak{D})$ dominate $\left(r_{1}, r_{2}^{*}\right)$. Since $\mathfrak{S}_{\mathfrak{O} w_{\mathfrak{E}}}$ is distinguished and symmetric and $w(\mathfrak{B})$ is not on the diagonal, it follows that $w(\mathfrak{D})>w(\mathfrak{B})$. This implies, since $w(\mathfrak{D})$ is on the diagonal, $w(\mathfrak{D})>p_{v}(\alpha)$. Since depth $\mathfrak{S}_{\mathfrak{O} w_{\mathfrak{E}}} w(\mathfrak{D})=d$ by Corollary 8.3.9, we are done.

(4) Let $x$ be an element of $\mathfrak{O} I(d)$ that $\mathfrak{O}$-dominates $\mathfrak{E}$. We will show that $x \geq \mathfrak{O} w_{\mathfrak{E}}$. By [7, Lemma 5.5], it is enough to show that $x$ dominates $\mathfrak{S}_{\mathfrak{O}} w_{\mathfrak{E}}$. By Lemma 8.1.3, it is enough to show the following: for every block $\mathfrak{B}$ of $\mathfrak{E}$, there exists $\beta$ in $\mathfrak{S}_{x}$ such that $\beta$ dominates $w(\mathfrak{B})$ and $\operatorname{depth}_{\mathfrak{S}_{x}} \beta \geq \operatorname{depth}_{\mathfrak{S}_{\mathfrak{O} w_{\mathfrak{E}}}} w(\mathfrak{B})$.

Let $\mathfrak{B}$ be a block of $\mathfrak{E}$. By Corollary 8.3.9, $\operatorname{depth}_{\mathfrak{S}_{\mathfrak{O} w_{\mathfrak{E}}}} w(\mathfrak{B})=\stackrel{k}{k}$ where $\mathfrak{B} \subseteq \mathfrak{O} \mathfrak{E}_{k}$. Let $\mathfrak{S}_{x}^{k}$ denote the set of elements of $\mathfrak{S}_{x}$ of depth at least $k$. Our goal is to show that there exists $\beta$ in $\mathfrak{S}_{x}^{k}$ that dominates $w(\mathfrak{B})$. Since $\mathfrak{S}_{x}$ is distinguished and $\mathfrak{B}$ is a block, it suffices to show the following: given $\alpha \in \mathfrak{B}$, there exists $\beta$ in $\mathfrak{S}_{x}^{k}$ (depending upon $\alpha$ ) that dominates $\alpha$. Moreover, since $\mathfrak{B}$ and $\mathfrak{S}_{x}^{k}$ are symmetric, we may assume that $\alpha=\alpha$ (up).

So now let $\alpha=\alpha$ (up) belong to $\mathfrak{B}$. Then either

(1) $\alpha$ belongs to $\mathfrak{O} \mathfrak{E}_{k}^{\mathrm{pr}}$, or

(2) $k$ is odd, $\mathfrak{E}$ is truly orthogonal at $k$, and $\alpha=p_{v}\left(\sigma_{k}\right)$, or

(3) $k$ is even, $\mathfrak{E}$ is truly orthogonal at $k-1$, and $\alpha=p_{h}\left(\sigma_{k-1}\right)$.

The proofs in the three cases are similar. In the first case, choose a $v$-chain $C$ in $\mathfrak{E}$ with tail $\alpha$ such that $\mathfrak{O}-\operatorname{depth}_{C}(\alpha)=k$ (see Corollary 5.2 .3 (1)). Then $\operatorname{depth}_{\mathfrak{O} \mathfrak{m o n}_{C}} \mathfrak{O} q_{C, \alpha}=k$ and, clearly, $\mathfrak{O} q_{C, \alpha}$ dominates $\alpha$. Since $x$ dominates 
$\mathfrak{O m o n}{ }_{C}$, there exists, by Lemma 4.5 of [7], $\beta$ in $\mathfrak{S}_{x}^{k}$ that dominates $\mathfrak{D} q_{C, \alpha}$ (and so also $\alpha$ ).

In the second case, choose a $v$-chain $C$ in $\mathfrak{E}$ with tail $\sigma_{k}$ with the property that $\mathfrak{O}$-depth ${ }_{C}\left(\sigma_{k}\right)=k$. Then depth $\mathfrak{O m o n}_{C} \mathfrak{O} q_{C, \sigma_{k}}=k$. Since $p_{h}\left(\sigma_{k}\right)$ belongs to $\mathfrak{N}(v)$, $\sigma_{k}$ is of type $\mathrm{V}$ or $\mathrm{H}$ in $C$, so $\mathfrak{D} q_{C, \sigma_{k}}=\alpha$. Since $x$ dominates $\mathfrak{O m o n} C$, there exists, by [7, Lemma 4.5], $\beta$ in $\mathfrak{S}_{x}^{k}$ that dominates $\mathfrak{O} q_{C, \sigma_{k}}=\alpha$.

In the third case, choose a $v$-chain $C$ in $\mathfrak{E}$ with tail $\sigma_{k-1}$ such that the $\mathfrak{O}$-depth in $C$ of $\sigma_{k-1}$ is $k-1$. Then depth $\mathfrak{O m o n}_{C} \mathfrak{O} q_{C, \sigma_{k-1}}=k-1$. Since $p_{h}\left(\sigma_{k-1}\right)$ belongs to $\mathfrak{N}(v), \sigma_{k-1}$ is of type $\mathrm{V}$ or $\mathrm{H}$ in $C$, so $\mathfrak{O} q_{C, \sigma_{k-1}}=p_{v}\left(\sigma_{k-1}\right)$. From Lemma 5.3.2 (3), it follows, since $k-1$ is odd, that $\sigma_{k-1}$ is of type H. Since $p_{v}\left(\sigma_{k-1}\right)>p_{h}\left(\sigma_{k-1}\right)=\alpha$, it follows that depth $\mathfrak{O m o n}_{C} p_{h}\left(\sigma_{k-1}\right) \geq k$ (in fact equality holds as is easily seen). Since $x$ dominates $\mathfrak{O m o n}_{C}$, there exists, by [7, Lemma 4.5], $\beta$ in $\mathfrak{S}_{x}^{k}$ that dominates $p_{h}\left(\sigma_{k-1}\right)=\alpha$.

\subsection{Proof that $\mathfrak{O} \phi \mathfrak{O} \pi=$ identity}

Let $\mathfrak{E}$ be a monomial in $\mathfrak{O N}(v)$ and let $\mathfrak{O} \pi=\left(\mathfrak{O} w_{\mathfrak{E}}, \mathfrak{O} \Pi_{\mathfrak{E}}\right)$. We need to show that $\mathfrak{O} \phi$ applied to the pair $\left(\mathfrak{O} w_{\mathfrak{E}}, \mathfrak{O} \Pi_{\mathfrak{E}}\right)$ gets us back to $\mathfrak{E}$. We know from (3) of Proposition 4.3.1 that $\mathfrak{O} w_{\mathfrak{E}} \mathfrak{O}$-dominates $\mathfrak{O} \Pi_{\mathfrak{E}}$, so $\mathfrak{O} \phi$ can indeed be applied to the pair $\left(\mathfrak{O} w_{\mathfrak{E}}, \mathfrak{O} \Pi_{\mathfrak{E}}\right)$.

The main ingredients of the proof are the corresponding assertion in the case of Grassmannian [7, Proposition 4.2] and the following claim which we will presently prove:

$$
\left.\mathfrak{O}\left(\mathfrak{O} \Pi_{\mathfrak{E}}\right)_{w, j, j+1}=\mathfrak{O \mathfrak { E } _ { j } ^ { \prime }}{ }_{j} \text { up }\right) \cup \mathfrak{O \mathfrak { E } _ { j + 1 } ^ { \prime }} \text { (up) } \quad \text { for every odd integer } j
$$

Let us first see how the assertion follows assuming the truth of the claim, by tracing the steps involved in applying $\mathfrak{O} \phi$ to $\left(\mathfrak{O} w_{\mathfrak{E}}, \mathfrak{O} \Pi_{\mathfrak{E}}\right)$. From the claim it follows that when we partition $\mathfrak{O} \prod_{\mathfrak{E}}$ into subsets (as a first step in the application of $\mathfrak{O} \phi-$ see $\S 7$ ), we get $\mathfrak{O} \mathfrak{E}_{j}^{\prime}$ (up) $\cup \mathfrak{O} \mathfrak{E}_{j+1}^{\prime}$ (up) (for odd integers $j$ ). Adding the mirror images will

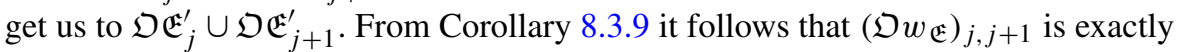
the element of $I(d, 2 d)$ obtained by acting $\pi$ on $\mathfrak{D E}_{j} \cup \mathfrak{D E} \mathfrak{E}_{j+1}$. Now, since $\phi \circ \pi=$ identity, it follows that on application of $\phi$ to $\left.\left(\mathfrak{O} w_{\mathfrak{E}}\right)_{j, j+1}, \mathfrak{\mathcal { E }} \mathfrak{E}_{j}^{\prime} \cup \mathfrak{O} \mathfrak{E}_{j+1}^{\prime}\right)$ we obtain $\mathfrak{O E} \mathfrak{E}_{j} \cup \mathfrak{O E} \mathfrak{E}_{j+1}$. By twisting the two diagonal elements in $\mathfrak{O E} \mathfrak{E}_{j} \cup \mathfrak{O E} \mathfrak{E}_{j+1}$ (if they exist at all) and removing the elements below the diagonal $\mathfrak{d}(v)$, we get back $\mathfrak{O} \mathfrak{E}_{j, j+1}^{\text {pr }}$. Taking the union of $\mathfrak{O} \mathfrak{E}_{j, j+1}^{\mathrm{pr}}$ (over odd integers $j$ ), we get back $\mathfrak{E}$.

Thus we need only prove the claim. Since $\mathfrak{O} \Pi_{\mathfrak{E}}$ is the union over all odd integers of the right hand sides (this follows from the definition of $\mathfrak{O} \Pi_{\mathfrak{E}}$ ), and the left hand sides as $j$ varies are mutually disjoint, it is enough to show that the right hand side is contained in the left hand side. Thus we need only prove: for $j$ an odd integer and $\alpha$ an element in $\mathfrak{O} \mathfrak{E}_{j}^{\prime}$ (up) $\cup \mathfrak{O} \mathfrak{E}_{j+1}^{\prime}$ (up),

- every $v$-chain in $\mathfrak{O} \Pi_{\mathfrak{E}}$ with head $\alpha$ is $\mathfrak{O}$-dominated by $\left(\mathfrak{O} w_{\mathfrak{E}}\right)^{j}$.

- there exists a $v$-chain in $\mathfrak{O} \Pi_{\mathfrak{E}}$ with head $\alpha$ that is not $\mathfrak{O}$-dominated by $\left(\mathfrak{O} w_{\mathfrak{E}}\right)^{j+2}$.

To prove the first item, write $\mathfrak{T}=\mathfrak{O E}^{j, j+1}:=\{\alpha \in \mathfrak{E} \mid \mathfrak{O}$-depth $\mathfrak{E}(\alpha) \geq j\}$ and set $\mathfrak{O} \pi(\mathfrak{T})=(x, \mathfrak{U})$. By Proposition 5.3.7, we have $\mathfrak{O T}_{i}^{\mathrm{pr}} \cup \mathfrak{O T} \mathfrak{T}_{i+1}^{\mathrm{pr}}=\mathfrak{O} \mathfrak{E}_{i+j-1}^{\mathrm{pr}} \cup \mathfrak{O} \mathfrak{E}_{i+j}^{\mathrm{pr}}$ 
for any odd integer $i$. Thus, by the description of $\mathfrak{O} \pi$, we have $\mathfrak{U}=\cup_{k \geq j} \mathfrak{O} \mathfrak{E}_{k}^{\prime}$ (up). By Corollary 8.3.9 and the description of $\mathfrak{O} \pi$, we have $x=\left(\mathfrak{O} w_{\mathfrak{E}}\right)^{j}$. By Corollary 8.3.10, any $v$-chain in $\mathfrak{O} \Pi_{\mathfrak{E}}$ with head belonging to $\mathfrak{O \mathfrak { E } _ { j } ^ { \prime }}{ }_{j}$ (up) $\cup \mathfrak{O} \mathfrak{E}_{j+1}^{\prime}$ (up) is contained entirely in $\cup_{k \geq j} \mathfrak{O} \mathfrak{E}_{k}^{\prime}$ (up). Finally, by Proposition 4.3.1 (3) applied to $\mathfrak{T}$, the desired conclusion follows.

To prove the second item we use Lemma 8.4.3. Proceed by decreasing induction on $j$. For $j$ sufficiently large the assertion is vacuous, for $\mathfrak{O \mathfrak { E } _ { j } ^ { \prime }}$ (up) $\cup \mathfrak{O \mathfrak { E } _ { j + 1 } ^ { \prime }}$ (up) is empty. To prove the induction step, assume that the assertion holds for $j+2$. If the $v$-chain consisting of the single element $\alpha$ is not $\mathfrak{O}$-dominated by $\left(\mathfrak{O} w_{\mathfrak{E}}\right)^{j+2}$, then we are done. So let us assume the contrary. Since the $\mathfrak{O}$-depth of the singleton $v$-chain $\alpha$ is at most 2, it follows from Lemma 8.2.2 that $\left(\mathfrak{O} w_{\mathfrak{E}}\right)_{j+2, j+3} \mathfrak{O}$-dominates the $v$-chain $\alpha$. Apply Lemma 8.4.3 with $k=j+2$. By its conclusion, either there exists $\beta \in \mathfrak{O} \mathfrak{E}_{j+2}^{\prime}$ (up) such that $\alpha>\beta$ or there exists $\gamma \in \mathfrak{O} \mathfrak{E}_{j+3}^{\prime}$ (up) such that $p_{h}(\alpha)>\gamma$.

First suppose that a $\gamma$ as above exists. By induction, there exists a $v$-chain in $\mathfrak{O} \Pi_{\mathfrak{E}}$ - call it $D$-with head $\gamma$ that is not $\mathfrak{O}$-dominated by $\left(\mathfrak{O} w_{\mathfrak{E}}\right)^{j+4}$. Let $C$ be the concatenation of $\alpha>\gamma$ and $D$. Since elements of $D$ have $\mathfrak{O}$-depth at least 3 in $C$ (Lemma 5.3.2 (1)), it follows from Corollary 8.2.3 that $C$ is not $\mathfrak{O}$-dominated by $\left(\mathfrak{O} w_{\mathfrak{E}}\right)^{j+2}$, and we are done.

Now suppose that such a $\gamma$ does not exist. Then a $\beta$ as above exists. If $\alpha>\beta$ is not $\mathfrak{O}$-dominated by $\left(\mathfrak{O} w_{\mathfrak{E}}\right)^{j+2}$ we are again done. So assume the contrary. Since the $\mathfrak{O}$ depth of $\beta$ in $\alpha>\beta$ is at least 2 , it follows that there exists an element of $\left(\mathfrak{S}_{\mathfrak{O} w_{\mathfrak{E}}}\right)_{j+3}$ that dominates $\beta$. Applying Lemma 8.4.3 again, this time with $k=j+3$, we find $\gamma^{\prime} \in \mathfrak{O E _ { j + 3 } ^ { \prime }}$ (up) such that $\beta>\gamma^{\prime}$. Arguing as in the previous paragraph with $\gamma^{\prime}$ in place of $\gamma$, we are done.

\subsection{Proof that $\mathfrak{O} \pi \mathfrak{O} \phi=$ identity}

Let $\mathfrak{T}$ be a monomial in $\mathfrak{O N}(v)$ and $w$ an element of $\mathfrak{O} I(d)$ that $\mathfrak{O}$-dominates $\mathfrak{T}$. We can apply $\mathfrak{O} \phi$ to the pair $(w, \mathfrak{T})$ to obtain a monomial $\mathfrak{O} \phi(w, \mathfrak{T})$ in $\mathfrak{O N}(v)$. We need to show that $\mathfrak{O} \pi$ applied to $\mathfrak{O} \phi(w, \mathfrak{T})$ results in $(w, \mathfrak{T})$. The main step of the proof is to establish the following:

$$
\mathfrak{O T} \mathfrak{T}_{w, j, j+1}^{\star}=\mathfrak{O}(\mathfrak{O} \phi(w, \mathfrak{T}))_{j, j+1}^{\mathrm{pr}}
$$

(for the meaning of the left and right sides of the above equation, see $\$ 7$ and $\$ 6$ respectively). Assuming this for the moment let us show that $\mathfrak{O} \pi \circ \mathfrak{O} \phi=$ identity.

We trace the steps involved in applying $\mathfrak{O} \pi$ to $\mathfrak{O} \phi(w, \mathfrak{T})$. From Eq. (9.3.1) it follows that when we break up $\mathfrak{O} \phi(w, \mathfrak{T})$ according to the $\mathfrak{O}$-depths of its elements as in $\S 6$, we get $\mathfrak{D} \mathfrak{T}_{w, j, j+1}^{\star}$ (as $j$ varies over odd integers). The next step in the application of $\mathfrak{O} \pi$ is the passage from $\mathfrak{O}(\mathfrak{O} \phi(w, \mathfrak{T}))_{j, j+1}^{\mathrm{pr}}$ to $\mathfrak{O}(\mathfrak{O} \phi(w, \mathfrak{T}))_{j, j+1}$. This involves replacing $\sigma_{j}$ by its projections and adding the mirror image of the remaining elements of $\mathfrak{O}(\mathfrak{O} \phi(w, \mathfrak{T}))_{j, j+1}^{\mathrm{pr}}$. It follows from Proposition 7.2.2 (3) that $\sigma_{j}=\delta_{j}$ and so $\mathfrak{O}(\mathfrak{D} \phi(w, \mathfrak{T}))_{j, j+1}=\left(\mathfrak{D T} \mathfrak{T}_{w, j, j+1} \cup \mathfrak{O T} \mathfrak{T}_{w, j, j+1}^{\#}\right)^{\star}$. The next step is to apply $\pi$ to $\left(\mathfrak{D T} \mathfrak{T}_{w, j, j+1} \cup \mathfrak{O} \mathfrak{T}_{w, j, j+1}^{\#}\right)^{\star}$. Since $\pi$ is the inverse of $\phi$ (as proved in [7]), we have $\pi\left(\left(\mathfrak{D T} \mathfrak{T}_{w, j, j+1} \cup \mathfrak{D T} \mathfrak{T}_{w, j, j+1}^{\#}\right)^{\star}\right)=\left(w_{j, j+1}, \mathfrak{D T} \mathfrak{T}_{w, j, j+1}\right)$. Since $\mathfrak{S}_{w}$ and $\mathfrak{T}$ are 
respectively the unions, as $j$ varies over odd integers, of $\left(\mathfrak{S}_{w}\right)_{j, j+1}$ and $\mathfrak{O T} \mathfrak{T}_{w, j, j+1}$, we see that $\mathfrak{O} \pi$ applied to $\mathfrak{O} \phi(w, \mathfrak{T})$ results in $(w, \mathfrak{T})$.

Thus it remains only to establish Eq. (9.3.1). It is enough to show that the left hand side is contained in the right hand side, for the union over all odd $j$ of either side is $\mathfrak{O} \phi(w, \mathfrak{T})$ and the right hand side is moreover a disjoint union. In other words, we need only show that the $\mathfrak{O}$-depth in $\mathfrak{O} \phi(w, \mathfrak{T})$ of an element of $\mathfrak{D T} \mathfrak{T}_{w, j, j+1}^{\star}$ is either $j$ or $j+1$. We will show, more precisely, that, for any element $\beta$ of $\mathfrak{S}_{w}$, the $\mathfrak{D}$ depth in $\mathfrak{O} \phi(w, \mathfrak{T})$ of any element of $\mathfrak{D}(\mathfrak{T}, w, \beta)^{\star}$ equals the depth in $\mathfrak{S}_{w}$ of $\beta$. Lemma 8.4.4 will be used for this purpose.

Let $\alpha$ be an element of $\mathfrak{O}(\mathfrak{T}, w, \beta)^{\star}$ and set $e:=\mathfrak{O}$-depth $\operatorname{OQ}_{\phi(w, \mathfrak{T})}(\alpha)$. We first show, by induction on $d:=\operatorname{depth}_{\mathfrak{S}_{w}} \beta$, that $e \geq d$. There is nothing to prove in case $d=1$, so we proceed to the induction step. Let $\beta^{\prime}$ be the element of $\mathfrak{S}_{w}$ of depth $d-1$ such that $\beta^{\prime}>\beta$. If there exists $\alpha^{\prime}$ in $\mathfrak{D P}_{\beta^{\prime}}^{\star}$ with $\alpha^{\prime}>\alpha$, the desired conclusion follows from Corollary 5.2.3 (3) and induction. Lemma 8.4.4 says that such an $\alpha^{\prime}$ exists in case $d$ is even. So suppose that $d$ is odd and such an $\alpha^{\prime}$ does not exist. The same lemma now says that $p_{h}\left(\delta_{d-2}\right)>\alpha$, so the desired conclusion follows from Lemma 5.3.2 (1).

We now show, by induction on $e$, that $d \geq e$. There is nothing to prove in case $e=1$, so we proceed to the induction step. Let $C$ be a $v$-chain in $\mathfrak{O} \phi(w, \mathfrak{T})$ with tail $\alpha$ and having the good property of Proposition 5.3.4. Let $\alpha^{\prime}$ be the immediate predecessor in $C$ of $\alpha$. Let $\beta^{\prime}$ in $\mathfrak{S}_{w}$ be such that $\alpha^{\prime} \in \mathfrak{O P}_{\beta^{\prime}}^{*}$ (we are not claiming at the moment that $\beta^{\prime}$ is unique although that is true and follows from the assertion that we are proving, the distinguishedness of $\mathfrak{S}_{w}$, and the fact that $\beta^{\prime}$ dominates $\alpha^{\prime}$ ). It follows from Corollary 8.4.6 that $\beta^{\prime}>\beta$.

Let $d^{\prime}:=\operatorname{depth}_{\mathfrak{S}_{w}} \beta^{\prime}$. It follows from Corollary 5.2.3 (3) that $e^{\prime}<e$ where $e^{\prime}:=$ $\mathfrak{O}$-depth $\mathfrak{O} \phi(w, \mathfrak{T})\left(\alpha^{\prime}\right)$. We have, $d \geq d^{\prime}+1 \geq e^{\prime}+1 \geq(e-2)+1=e-1$, the first equality being justified because $\beta^{\prime}>\beta$, the second by the induction hypothesis, and the last by Lemma 5.3.2 (1). It suffices to rule out the possibility that $d=e-1$. So assume $d=e-1$. Then $d=d^{\prime}+1$ and $d^{\prime}=e^{\prime}=e-2$. It follows from (1) of Lemma 5.3.2 that the $v$-chain $\alpha^{\prime}>\alpha$ has $\mathfrak{O}$-depth 3 and from (3) of the same lemma that $e^{\prime}$ is odd. But then we get a contradiction to Proposition 7.2.2 (2) ( $\alpha^{\prime}$ and $\alpha$ belong to $\mathfrak{O T}_{w, d^{\prime}, d^{\prime}+1}^{\star}$ ). The proof of Eq. (9.3.1) is thus over.

\subsection{Proof of Proposition 4.3.3}

Observe that the condition ( $\ddagger)$ makes sense also for a monomial of $\mathfrak{N}(v)$. By virtue of belonging to $\mathfrak{O} I(d), v$ has $f^{*}$ as an entry. It follows from the description of the bijection $w \leftrightarrow \mathfrak{S}_{w}$ of $\S 2.6 .2$ that for an element $w$ of $\mathfrak{O} I(d)$ to satisfy (†) it is necessary and sufficient that $\mathfrak{S}_{w}$ (equivalently all its parts $\left(\mathfrak{S}_{w}\right)_{j, j+1}=: \mathfrak{S}_{w, j, j+1}$ satisfy $(\ddagger)$.

(1) Since $\mathfrak{T}$ satisfies $(\ddagger)$, so do its parts $\mathfrak{D T} \mathfrak{T}_{w, j, j+1}$ and $\mathfrak{O T} \mathfrak{T}_{w, j, j+1} \cup \mathfrak{D} \mathfrak{T}_{w, j, j+1}^{\#}$ (adding the mirror image preserves $(\ddagger)$ ). Since $\mathfrak{S}_{w, j, j+1}$ also satisfies $(\ddagger)$, it follows from the description of the map $\phi$ of [7] (observe the passage from a piece $\mathfrak{P}$ to its 'star' $\left.\mathfrak{P}^{*}\right)$ that the $\left(\mathfrak{D T} w, j, j+1 \cup \mathfrak{O} \mathfrak{T}_{w, j, j+1}^{\#}\right)^{\star}$ satisfy $(\ddagger)$. Since the 'twisting' involved in the passage from $\left(\mathfrak{D T} \mathfrak{T}_{w, j, j+1} \cup \mathfrak{D} \mathfrak{T}_{w, j, j+1}^{\#}\right)^{\star}$ to $\mathfrak{D T} \mathfrak{T}_{w, j, j+1}^{\star}$ involves only a rearrangement of row and column indices, it follows that the $\mathfrak{D T}_{w, j, j+1}^{\star}$ satisfy $(\ddagger)$. Finally so also does their union $\mathfrak{O} \phi(w, \mathfrak{T})$. 
(2) The parts $\mathfrak{O} \mathfrak{E}_{j, j+1}^{\mathrm{pr}}$ of $\mathfrak{E}$ clearly satisfy ( $\left.\ddagger\right)$. Therefore so do the $\mathfrak{O} \mathfrak{E}_{j, j+1}$, for, first of all, adding the mirror image preserves ( $\ddagger)$, and then the removal of $\sigma_{j}$ and addition of its projections involves only a rearrangement of row and column indices. It follows from description of the map $\pi$ of [7] (observe the passage from a block $\mathfrak{B}$ to the pair $\left.\left(w(\mathfrak{B}), \mathfrak{B}^{\prime}\right)\right)$ that both $\left(\mathfrak{O} w_{\mathfrak{E}}\right)_{j, j+1}$ and $\mathfrak{O} \mathfrak{E}_{j, j+1}^{\prime}$ satisfy ( $\left.\ddagger\right)$. Finally, $\mathfrak{S}_{\mathfrak{O} w_{\mathfrak{E}}}$ and $\mathfrak{O} \Pi_{\mathfrak{E}}$ being the union (respectively) of $\left(\mathfrak{S}_{\mathfrak{O} w_{\mathfrak{E}}}\right)_{j, j+1}$ and $\mathfrak{O} \mathfrak{E}_{j, j+1}^{\prime}$ (up), they satisfy ( $\ddagger)$.

\section{References}

1. Brion, M., Polo, P.: Generic singularities of certain Schubert varieties. Math. Z. 231(2), 301-324 (1999)

2. De Negri, E.: Some results on Hilbert series and $a$-invariant of Pfaffian ideals. Math. J. Toyama Univ. 24, 93-106 (2001)

3. Ghorpade, S.R., Krattenthaler, C.: The Hilbert series of Pfaffian rings. In: Algebra, arithmetic and geometry with applications, pp. 337-356. West Lafayette, IN, 2000, Springer, Berlin (2004)

4. Ghorpade, S.R., Raghavan, K.N.: Hilbert functions of points on Schubert varieties in the Symplectic Grassmannian. Trans. Am. Math. Soc. 358, 5401-5423 (2006)

5. Herzog, J., Trung, N.V.: Gröbner bases and multiplicity of determinantal and Pfaffian ideals. Adv. Math. 96(1), 1-37 (1992)

6. Ikeda, T., Naruse, H.: Excited Young diagrams and equivariant Schubert calculus. Trans. Am. Math. Soc. Electronically published on April 30 (2009). arXiv:math/0703637

7. Kodiyalam, V., Raghavan, K.N.: Hilbert functions of points on Schubert varieties in Grassmannians. J. Algebra 270(1), 28-54 (2003)

8. Krattenthaler, C.: On multiplicities of points on Schubert varieties in Grassmannians. II. J. Algebraic Combin. 22(3), 273-288 (2005)

9. Kreiman, V.: Monomial bases and applications for Schubert and Richardson varieties in ordinary and affine Grassmannians. Ph.D. Thesis, Northeastern University (2003)

10. Kreiman, V.: Local properties of Richardson varieties in the Grassmannian via a bounded Robinson-Schensted-Knuth correspondence. J. Algebraic Combin. 27(3), 351-382 (2008) arXiv:math.AG/0511695

11. Kreiman, V., Lakshmibai, V.: Multiplicities of singular points in Schubert varieties of Grassmannians. In: Algebra, arithmetic and geometry with applications, West Lafayette, IN, 2000, pp. 553-563. Springer, Berlin (2004)

12. Kreiman, V., Lakshmibai, V.: Richardson varieties in the Grassmannian. In: Contributions to automorphic forms, geometry, and number theory, pp. 573-597. Johns Hopkins Univ. Press, Baltimore (2004)

13. Lakshmibai, V., Seshadri, C.S.: Geometry of $G / P$. II. The work of de Concini and Procesi and the basic conjectures. Proc. Indian Acad. Sci. Sect. A 87(2), 1-54 (1978)

14. Lakshmibai, V., Seshadri, C.S.: Geometry of G/P. V. J. Algebra 100(2), 462-557 (1986)

15. Lakshmibai, V., Weyman, J.: Multiplicities of points on a Schubert variety in a minuscule $G / P$. Adv. Math. 84(2), 179-208 (1990)

16. Littelmann, P.: Contracting modules and standard monomial theory for symmetrizable Kac-Moody algebras. J. Amer. Math. Soc. 11(3), 551-567 (1998)

17. Raghavan, K.N., Sankaran, P.: A new approach to standard monomial theory for classical groups. Transform. Groups 3(1), 57-73 (1998)

18. Raghavan, K.N., Upadhyay, S.: Initial ideals of tangent cones to Schubert varieties in orthogonal Grassmannians. J. Combin. Theory Ser. A 116(3), 663-683 (2009). arXiv:0710.2950

19. Seshadri, C.S.: Geometry of $G / P$. I. Theory of standard monomials for minuscule representations. In: C.P. Ramanujam - a tribute. Tata Inst. Fund. Res. Studies in Math., vol. 8, pp. 207-239. Springer, Berlin (1978)

20. Upadhyay, S.: Schubert varieties in the Orthogonal Grassmannian. Ph.D. Thesis, Chennai Mathematical Institute (2008). http://www.imsc.res.in/ knr 